\title{
When to Adopt a Service Innovation: Nash Equilibria in a Competitive Diffusion Framework
}

\author{
M. Fazıl Paç \\ Google Inc., 1600 Amphitheatre Parkway, Mountain View, CA 94043fazil@google.com, \\ Sergei Savin (Corresponding Author) \\ Wharton School, University of Pennsylvania, Philadelphia, PA 19104, savin@wharton.upenn.edu, \\ Chander Velu \\ Department of Engineering, University of Cambridge, UK, CB3 0FS, c.velu@eng.cam.ac.uk,
}

We study the optimal timing of adoption of a service innovation that a new entrant firm brings to a market populated by two incumbent firms. Our analysis is based on a model of competitive diffusion dynamics that extends the monopolistic Bass model to include customer churn processes, as well as a potential market expansion resulting from the introduction of the innovation. We obtain expressions for the time trajectories of the customer bases, i.e., the numbers of customers that use old and new service processes for the competing firms in a general setting, as well as sharper, closed-form characterizations for the setting with a stable market and homogeneous imitation process.

In modeling competitive dynamics we consider settings where incumbents anticipate a potential failure of the innovation. We use the trajectories for the customer bases to model an optimal adoption response problem faced by one of the incumbent firms in the setting in which the adoption time for the other incumbent can be anticipated or is pre-announced, and analyze this problem in the absence of market expansion or intra-generational customer churn. Using the optimal response results, we provide the Nash equilibrium analysis of the adoption decisions by competing incumbent firms and derive sufficient conditions for the "now-now", "now-never" and "never-never" adoption equilibria. We use the trading volume data from the foreign exchange markets to estimate the parameters of the competitive diffusion dynamics for our model and to conduct a numerical investigation of the impact of the uncertainty associated with the success of the innovation on the incumbents' Nash equilibrium adoption times.

Key words: OR in Marketing, Optimal Adoption Timing, Nash Equilibria, Diffusion Models 


\section{Introduction}

Innovations driven by new technologies are being introduced at increasing speed (Ringel et al. 2015). The shorter life-cycle times underscore the importance for firms to appropriately time their innovation adoption decisions. Multiple studies have shown that an innovation adoption time is an important marketing decision, which can determine the success of the innovation (Golder and Tellis 1993; Shankar et al. 1998; Frattini et al. 2013; Bhargava et al. 2013). In the present work, we consider a service setting in which a market entrant firm, leveraging a new technology to launch an innovation, enters the market populated by two incumbent firms. We use the term "customer base" to denote the numbers of customer accounts that, at a given time, use a particular firmbusiness model combination. The incumbents with differing initial customer bases need to respond by deciding when to adopt the innovation. Such a setting is commonly found in many industries, and it has long been observed that some incumbent firms are quick to adopt an innovation while others appear to be very slow.

For example, incumbent firms adopted online business models at different times in the equity brokerage market and the foreign exchange market in the US. Charles Schwab, the major firm in equity brokerage market, adopted the online brokerage model before the minor firm, Ameritrade. In contrast, Atriax, formed by the consortium of major banks in the foreign exchange market adopted an online foreign exchange model only after the minor banks adopted such a model via a consortium called FXAll. These contrasting observations about the timing of adoption of innovation by incumbents raise the question about the underlying reasons that drive the innovation adoption decision.

The issue of innovation adoption in a competitive setting is the focus of a number of papers in the economics literature. Benoit (1985) argues that the time when the incumbent firms adopt innovation depends on the expected profits that the innovation brings: the greater the expected profits from innovation, the earlier the adoption. However, the greater expected profits also increase the likelihood of adoption by rival firms, which might decrease the expected gains. These forces 
work in opposite directions and the net result might depend on one firm's evaluation of the other firm's response.

Several studies have also shown how uncertainty regarding the profitability of an innovation affects the adoption decision (Doraszelski 2004; Jensen 1988; Mamer and McCardle 1987; McCardle 1984). As argued in Rogers (1962), innovation adoption is often a contagious process due to the presence of strong imitation effects. Furthermore, it has been argued that contagion effects could be strengthened by the potential for substitution of the existing market by the market created by the innovation (King and Tucci 2002) or by a gradual reduction in uncertainty about the attractiveness of the innovation (Gilbert and Lierberman 1987; Haunschild and Miner 1997). In addition, several studies have shown that the introduction of an innovation could increase the size of the potential market, which in turn might affect the speed of response by incumbent firms (Golder and Tellis 1993; Sorescu et al. 2003; Aboulnasr et al. 2008)

All the papers described above consider static adoption settings. In particular, none of the above studies provide a detailed analytical account of customer adoption dynamics and how this might affect firms' profits and influence innovation adoption decisions. On the other hand, the marketing literature contains a well-developed stream of papers, starting with the seminal work of Bass (1969), focusing on the dynamics of innovation adoption by customers.

The literature on the diffusion of products and services in competitive settings has seen significant growth in recent years (Libai et al. 2009a; Libai et al. 2009b; Guseo and Montarino 2012; Guseo and Montarino 2014; Guseo and Montarino 2015). While a review paper by Peres et al. (2010) calls for an increased focus on the analysis of the optimal market entry timing, the literature on when to adopt an innovation or launch a new product in a competitive setting remains relatively sparse. Savin and Terwiesch (2005) and Guseo and Montarino (2010) looked at the optimal market entry time by considering a two-firm competitive diffusion model to study the life-cycle market shares of competing firms. A model of the optimal market entry with a single incumbent is analyzed in Joshi et al. (2009). In the present analysis, we extend Savin and Terwiesch (2005) by 
considering simultaneous diffusion processes of old and new business models in a three-firm setting.

Our ultimate goal is to address the limitation of the extant literature and analyze the equilibria of innovation adoption decisions emerging under a setting with competitive diffusion dynamics. Given the complexity of the analysis of the optimal adoption timing decisions, we assume in our modeling, similar to Savin and Terwiesch (2005) and Joshi et al. (2009), that the marketing-mix variables, such as pricing and advertising, are predetermined. This assumption describes an environment where the innovation adoption decisions are made in the presence of the independently set marketing-mix levels. Our diffusion model includes the intra-firm and inter-firm imitation processes in a Bass-like setting and increases in market potential for both the existing business model, as well as the new business model, upon the introduction of innovation (Mahajan et al. 1993; Krishnan et al. 2000). In addition, we incorporate intra-generational churn, whereby customers switch between firms within the same business model (Gupta et al. 2004; Libai et al 2009a).

Our modeling and the results of our analysis can be summarized as follows:

1. We extend the classical Bass-like approach to describe dynamics of adoption of a new service business model in a market populated by two incumbent firms and a single entrant firm. We consider a generalized setting in which the speed of the imitation adoption depends on whether the new business model is also offered by one of the incumbent firms or only by the entrant firm.

2. We provide a characterization of the diffusion trajectories of the customer bases for each competing firm and each service business model in the general setting and derive closed-form expressions in homogeneous and asymmetric imitation settings (Propositions 1-5). We also derive expressions for the terminal customer base values, which are often viewed as proxies for overall profits and for the strength of firms' competitive positions, both with and without intra-generational customer churn (Proposition 6). These values are expressed in terms of a few easy-to-estimate parameters. We also analyze the impact of the market expansion resulting from the introduction of the service model innovation and show that the incumbents may initially benefit from its introduction, as it attracts new potential customers for all competitors in the market. 
3. We model setting where the business model introduced by the entrant firm has a risk of failing and derive the closed-form expressions for the diffusion trajectories of the incumbent firms after potential failure of the service innovation (Proposition 7).

4. For the setting with asymmetric churn processes, we obtain closed-form expressions for the terminal values of customer base for the cases when the new service model fails as well as when it succeeds (Proposition 8).

5. The optimal adoption response problem faced by an incumbent firm is analyzed in a setting where the adoption time of the other incumbent can be anticipated or is preannounced. Using the expressions for the diffusion trajectories, we establish quasiconcavity of the firm's profit function (Proposition 9) and show that the optimal adoption response time is a non-decreasing function of the firm's initial profit-adjusted customer base values (Proposition 10) in the absence of market expansion or intra-generational customer churn.

6. For the same setting, we provide Nash equilibrium analysis of adoption decisions by competing incumbent firms and derive sufficient conditions for the realization of three adoption equilibria: immediate adoption by both incumbents, maximum-delay adoption by both incumbents, and immediate-delayed mixture of equilibrium adoption times (Proposition 11). We also show that in settings where the time discounting of profits is negligible, these equilibria are the only ones that can be observed.

7. We use the the FX market data, shown in Appendix B, to estimate the parameters of our model (Section 3) and apply the estimates to the numerical evaluation of the Nash equilibria in the new service model adoption times by the incumbents (Section 5).

Our paper is organized as follows. In the next section we introduce our model and derive expressions for the diffusion trajectories of the customer base values for each competing firm. Section 3 describes the analysis of the optimal adoption response decisions and the Nash equilibria adoption times. The implications of our findings and the directions for future research are discussed in Section 4 . 


\section{Competitive Diffusion Dynamics in the Presence of Innovation}

Consider a market populated by two incumbent firms (major incumbent and minor incumbent), which is faced (at $t=0$ ) with the entry of a new firm. In reality, the major incumbent and the minor incumbent firms can represent clusters of firms that form a consortia (e.g., top 3 vs. the rest of the market), which are similar in the way they approach the introduction of a new business model. We assume that the entrant firm employs only the new service model. In order to account for the uncertain, unproven nature of the service innovation and the possibility of its "failure", we assume that the new model fails with probability $P_{F} \in[0,1]$ and succeeds with probability $1-P_{F}$. In the case of success, the new firm (and the new service model) remains in the market at any $t \geq 0$. In the case of failure, the new entrant (and the new service model) remains on the market for $t \in\left[0, T_{F}\right]$, and then exits the market. We assume that the time-to-failure $T_{F}$, if failure occurs, is a random variable distributed on $[0,+\infty]$ according to the $\operatorname{CDF} \Phi(\cdot)$. In our analysis, we treat both the value of the failure probability $P_{F}$ as well as the distributional information describing the time-to-failure $T_{F}$ as known to both incumbents.

In our analysis, we assume that incumbents 1 and 2 commit, at time $t=0$, to adopting the innovation at $t=t_{1} \geq 0$ and $t=t_{2} \geq 0$, respectively, provided that the new entrant remains on the market at those times. We model the dynamics for the customer base for the old and the new service model using the classical framework proposed by Bass (1969) for describing adoption of durable goods and extended by Libai et al. (2009b) to include the diffusion of services.

The specific form of the customer base trajectory equations at any time $t$ depends on whether by this time the service innovation has been adopted by one (or both) of the incumbents.

In particular, the customer base dynamics proceeds through a set of distinct phases. For example, consider the case where the innovation is successful and the new entrant remains on the market for any positive $t$. Then, in phase I corresponding to the time period $\left[0, \min \left(t_{1}, t_{2}\right)\right]$, the entrant firm is the only one to employ the new service model. Phase II $\left(t \in\left[\min \left(t_{1}, t_{2}\right), \max \left(t_{1}, t_{2}\right)\right]\right)$ describes the competitive market-share dynamics in the period when the innovation has been adopted by 
only one of the incumbents. Next, in phase III $\left(t \in\left(\max \left(t_{1}, t_{2}\right),+\infty\right)\right)$ both incumbents employ the new service model. Note that, if the innovation fails, then the customer base dynamics will contain an additional phase that starts at time $t=T_{F}$. In this phase, which we call phase IV, both incumbents compete using the old service model.

Thus, at any time $t \geq 0$, the customer market base $\mathcal{M}$ can potentially be comprised of as many as 6 customer groups: two customer groups, $m_{1}^{\circ}(t)$ and $m_{2}^{\circ}(t)$, which continue to do business with one of the incumbent firms using the old service model, three customer groups, $m_{1}^{\mathrm{n}}(t), m_{2}^{\mathrm{n}}(t)$, and $m_{3}^{\mathrm{n}}(t)$, which switch to the new service model offered by either one of the incumbents (firms 1 and 2) or by the new entrant (firm 3), and the group of customers who have not yet adopted any service model.

Figure 1 provides an illustration of this market structure. Two features of the customer dynamics we model deserve a separate mention. First, in addition to the adoption of the new service model by customers who previously used the old one, we also consider the "churn" adoptions, that is, switching between firms within the same type of service model. This "churn" feature is common in the models of inter-generational product adoption and diffusion of services (Gupta et al. 2004; Libai et al. 2009a, Libai et al. 2009b, Peres et al. 2010). Second, we assume that the introduction of the new service model by a "newcomer" firm leads to the expansion in overall market size for both new and old models from $m_{1}^{\circ}(0)+m_{2}^{\circ}(0)$ to $\mathcal{M} \geq m_{1}^{\circ}(0)+m_{2}^{\circ}(0)$, with $\mathcal{M}-m_{1}^{\circ}(0)-m_{2}^{\circ}(0)$ representing the size of the market increase; in other words, the initial number of customers who have yet to adopt any service model. The increase in the overall market potential upon the introduction of innovation we use in our model is consistent with previous research on technological substitution and diffusion (Mahajan et al. 1993; Golder and Tellis 1993; Sorescu et al. 2003; Aboulnasr et al. 2008). The market potential expansion is modeled in the form of a one-off increase following the introduction of the innovation (Wilson and Norton 1989; Mahajan and Muller 1996; Krishnan et al. 2000).

Table 1 summarizes the notation we use in our model. 


\begin{tabular}{|l|l|}
\hline Notation & \multicolumn{1}{|c|}{ Description } \\
\hline$t_{1}$ & Innovation adoption time by the 1st incumbent \\
\hline$t_{2}$ & Innovation adoption time by the 2 st incumbent \\
\hline$P_{F}$ & Probability that the innovation fails \\
\hline$T_{F}$ & Time when the innovation fails, given the failure \\
\hline$m_{i}^{\mathrm{o}}(t)$ & Customer base of the "old" service model for incumbent $i=1,2$ at time $t$ \\
\hline$m_{i}^{\mathrm{n}}(t)$ & Customer base of the "new" service model for incumbent $i=1,2$ at time $t$ \\
\hline$m_{3}^{\mathrm{n}}(t)$ & Customer base of the "new" service model for the new entrant at time $t$ \\
\hline $\mathcal{M}$ & Total market potential \\
\hline$p_{\mathrm{o}}$ & Innovation coefficient for the "old" service model \\
\hline$q_{\mathrm{o}}$ & Imitation coefficient for the "old" service model \\
\hline$p_{\mathrm{oc}}$ & Innovation "churn" coefficient for the "old" service model \\
\hline$q_{\mathrm{oc}}$ & Imitation "churn" coefficient for the "old" service model \\
\hline$p_{\mathrm{n}}$ & Innovation coefficient for the "new" service model \\
\hline$q_{\mathrm{n}}$ & Imitation coefficient for the "new" service model offered by the new entrant \\
\hline$Q_{\mathrm{n}}$ & Imitation coefficient for the "new" service model offered by an incumbent \\
\hline$p_{\mathrm{nc}}$ & Innovation "churn" coefficient for the "new" service model \\
\hline
\end{tabular}

Table 1 Description of notation.

Below we conduct a detailed analysis of the competitive diffusion dynamics for each of the four adoption phases described above. We first consider the case where the new model succeeds and describe the dynamics of the first three phases of the market-share dynamics. Then, we add the description of the phase IV that appears if the new model fails.

\subsection{Phase I: "New Entrant Only"}

In this phase the new entrant is the only firm that uses the new service model. Let $t_{f}=\min \left(t_{1}, t_{2}\right)$. Then, at any time $t \in\left[0, t_{f}\right]$, the entire market $\mathcal{M}$ consists of four groups of customers: $m_{i}^{\circ}(t), i=1,2$ 


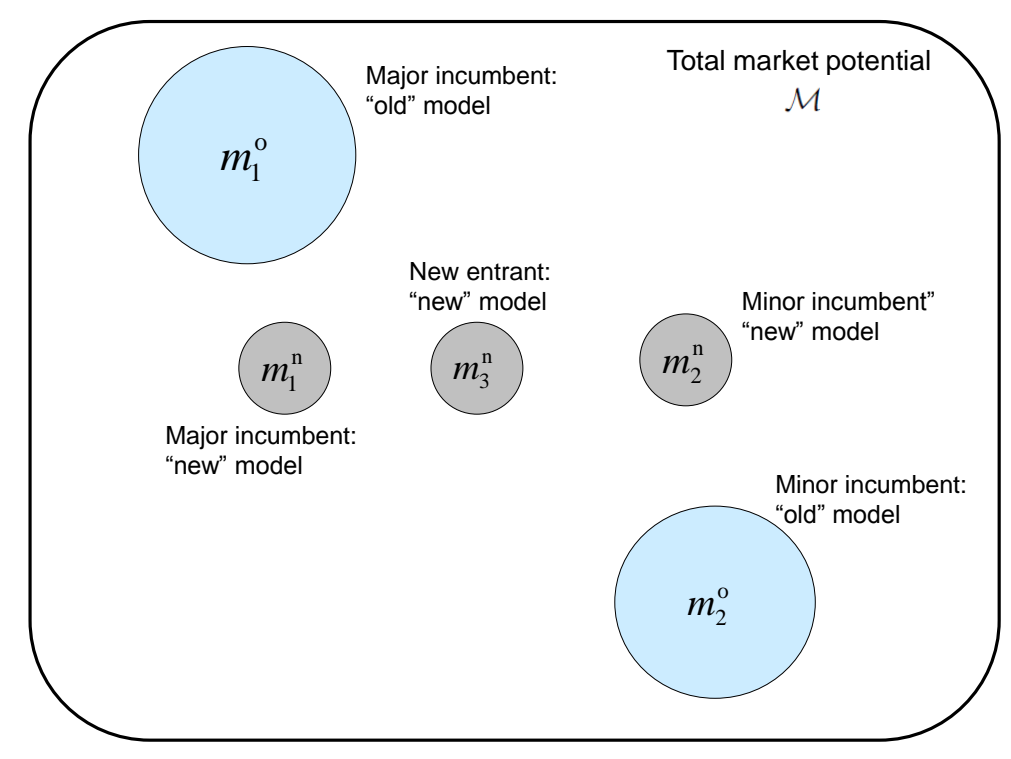

Figure 1 Customer groups in a competitive market

- those that continue to do business with the incumbent firm $i$ through the "old" service model; $m_{3}^{\mathrm{n}}(t)$ - those who opt to do business with the entrant firm; as well as those $\left(\mathcal{M}-m_{1}^{\circ}(t)-m_{2}^{\circ}(t)-\right.$ $\left.m_{3}^{\mathrm{n}}(t)\right)$ who have not yet adopted either service model. We assume that the new entrant operates exclusively through the new service model. We also assume that at the time when the innovation becomes available, the customer base for the major incumbent is higher than that for the minor incumbent: $m_{1}^{\circ}(0)=M>m=m_{2}^{\circ}(0)$. In addition, the initial customer base for the new entrant is 0 : $m_{3}^{\mathrm{n}}(0)=0$ (in other words, at $t=0$ the number of customers who have not yet adopted any business model is given by $\mathcal{M}-M-m)$. In describing the competitive diffusion dynamics, we will follow the diffusion approach widely used in the marketing literature to describe the adoption of innovations. In his seminal paper, Bass (1969) has applied contagion biological models to describe the process of adoption of durable products in a monopolistic setting. Since then, Bass' approach has been shown to be a reliable descriptor of innovation dynamics in many settings, and the definition of innovation was extended to include new services (Peres et al. 2010). We propose the following generalization of Bass diffusion dynamics for the competitive setting described above:

$$
\frac{d m_{1}^{\mathrm{o}}}{d t}=\left(p_{\mathrm{o}}+\frac{q_{\mathrm{o}}}{\mathcal{M}} m_{1}^{\mathrm{o}}\right)\left(\mathcal{M}-m_{1}^{\mathrm{o}}-m_{2}^{\mathrm{o}}-m_{3}^{\mathrm{n}}\right)
$$




$$
\begin{aligned}
& +\left(p_{\mathrm{oc}}+\frac{q_{\mathrm{oc}}}{\mathcal{M}} m_{1}^{\mathrm{o}}\right) m_{2}^{\mathrm{o}}-\left(p_{\mathrm{n}}+\frac{q_{\mathrm{n}}}{\mathcal{M}} m_{3}^{\mathrm{n}}+p_{\mathrm{oc}}+\frac{q_{\mathrm{oc}}}{\mathcal{M}} m_{2}^{\mathrm{o}}\right) m_{1}^{\mathrm{o}}, \\
\frac{d m_{2}^{\mathrm{o}}}{d t}= & \left(p_{\mathrm{o}}+\frac{q_{\mathrm{o}}}{\mathcal{M}} m_{2}^{\mathrm{o}}\right)\left(\mathcal{M}-m_{1}^{\mathrm{o}}-m_{2}^{\mathrm{o}}-m_{3}^{\mathrm{n}}\right) \\
& +\left(p_{\mathrm{oc}}+\frac{q_{\mathrm{oc}}}{\mathcal{M}} m_{2}^{\mathrm{o}}\right) m_{1}^{\mathrm{o}}-\left(p_{\mathrm{n}}+\frac{q_{\mathrm{n}}}{\mathcal{M}} m_{3}^{\mathrm{n}}+p_{\mathrm{oc}}+\frac{q_{\mathrm{oc}}}{\mathcal{M}} m_{1}^{\mathrm{o}}\right) m_{2}^{\mathrm{o}}, \\
\frac{d m_{3}^{\mathrm{n}}}{d t}= & \left(p_{\mathrm{n}}+\frac{q_{\mathrm{n}}}{\mathcal{M}} m_{3}^{\mathrm{n}}\right)\left(\mathcal{M}-m_{3}^{\mathrm{n}}\right) .
\end{aligned}
$$

Equation (1) describes the evolution of customer base of the "old" service model through the major incumbent in the presence of a new service model. Such evolution proceeds through two major channels: 1) increase through both innovation and imitation adoption processes from customers who were either using the old service model through the minor incumbent (the term $\left.\left(p_{\mathrm{oc}}+\frac{q_{\mathrm{oc}}}{\mathcal{M}} m_{1}^{\mathrm{o}}\right) m_{2}^{\circ}\right)$ or were not using any service model, old or new (the term $\left.\left(p_{\mathrm{o}}+q_{\mathrm{o}} m_{1}^{\mathrm{o}} / \mathcal{M}\right)\left(\mathcal{M}-m_{1}^{\mathrm{o}}-m_{2}^{\mathrm{o}}-m_{3}^{\mathrm{n}}\right)\right)$, and 2) decrease caused by customers switching over to the old service model provided by the minor incumbent (the term $\left.\left(p_{\text {oc }}+\frac{q_{\mathrm{oc}}}{\mathcal{M}} m_{2}^{\circ}\right) m_{1}^{\circ}\right)$ or to the innovation provided by the new entrant (the term $\left.\left(p_{\mathrm{n}}+q_{\mathrm{n}} m_{3}^{\mathrm{n}} / \mathcal{M}\right) m_{1}^{\mathrm{o}}\right)$. In $(1)$, innovation and imitation diffusion processes that result in the adoption of an old (new) service model are characterized by parameters $p_{\mathrm{o}}\left(p_{\mathrm{n}}\right)$ and $q_{\mathrm{o}}\left(q_{\mathrm{n}}\right)$, respectively. Similarly, the intra-generational churn is characterized by the innovation parameter $p_{\mathrm{oc}}$, and the imitation parameter $q_{\mathrm{oc}}$. The intra-generational churn innovation and imitation are not firm-specific and occur through symmetric coefficients. In other words, the churn adoption from firm 1's customer base into firm 2's service model occurs through identical innovation/imitation terms $\left(p_{\mathrm{oc}}\right.$ and $\left.q_{\mathrm{oc}}\right)$ with the churn adoption from firm 2's customer base into firm 1's service model. Note that the term describing intra-generational imitation from firm 2 to firm $1\left(\frac{q_{\text {oc }}}{\mathcal{M}} m_{1}^{\circ} m_{2}^{o}\right)$ is equal to the similar term describing intra-generational imitation from firm 1 to firm 2 . Hence, the churn-imitation terms $\left(\frac{q_{\mathrm{oc}}}{\mathcal{M}} m_{i}^{\circ} m_{j}^{\circ}\right)$ can be dropped from the diffusion equations, as we will do for the rest of the paper. Equation (2) describes the dynamics of the minor incumbent's customer base in a similar fashion, while equation (3) reflects the growth of the entrant firm's customer base (associated with the new business model). We note that equation (3) has the standard Bass form, which, subject to the initial condition $m_{3}^{\mathrm{n}}(0)=0$, produces a closed-form Bass-like solution:

$$
m_{3}^{\mathrm{n}}(t)=\mathcal{M}\left(1-\frac{q_{\mathrm{n}}+p_{\mathrm{n}}}{q_{\mathrm{n}}+p_{\mathrm{n}} e^{\left(q_{\mathrm{n}}+p_{\mathrm{n}}\right) t}}\right) .
$$


Equations (1)-(2) differ from the classical Bass form in a substantial way; yet, combined with initial conditions $m_{1}^{\circ}(0)=M$ and $m_{2}^{\circ}(0)=m(m+M<\mathcal{M})$ they also admit closed-form solutions. In order to describe these solutions, it is convenient to introduce the following notation. For a given set of diffusion parameters $p_{\mathrm{o}}, q_{\mathrm{o}}, p_{\mathrm{n}}$, and $q_{\mathrm{n}}$, define

$$
\begin{aligned}
\widehat{F}_{I}\left(p_{\mathrm{o}}, p_{\mathrm{n}}, q_{\mathrm{o}}, q_{\mathrm{n}}, t\right)= & { }_{2} F_{1}\left(\frac{2 p_{\mathrm{o}}+p_{\mathrm{n}}+q_{\mathrm{n}}}{q_{\mathrm{n}}+p_{\mathrm{n}}}, 1-\frac{q_{\mathrm{o}}}{q_{\mathrm{n}}} ; \frac{2\left(p_{\mathrm{o}}+p_{\mathrm{n}}+q_{\mathrm{n}}\right)}{q_{\mathrm{n}}+p_{\mathrm{n}}} ;-\frac{q_{\mathrm{n}}}{p_{\mathrm{n}}}\right)-e_{2}^{-\left(2 p_{\mathrm{o}}+p_{\mathrm{n}}+q_{\mathrm{n}}\right) t} \\
& \times{ }_{2} F_{1}\left(\frac{2 p_{\mathrm{o}}+p_{\mathrm{n}}+q_{\mathrm{n}}}{q_{\mathrm{n}}+p_{\mathrm{n}}}, 1-\frac{q_{\mathrm{o}}}{q_{\mathrm{n}}} ; \frac{2\left(p_{\mathrm{o}}+p_{\mathrm{n}}+q_{\mathrm{n}}\right)}{q_{\mathrm{n}}+p_{\mathrm{n}}} ;-\frac{q_{\mathrm{n}}}{p_{\mathrm{n}}} e^{-\left(p_{\mathrm{n}}+q_{\mathrm{n}}\right) t}\right),
\end{aligned}
$$

where ${ }_{2} F_{1}(a, b ; c ; x)$ is the hypergeometric function:

$$
\begin{aligned}
{ }_{2} F_{1}(a, b ; c ; x) & =1+\frac{a b}{1 ! c} x+\frac{a(a+1) b(b+1)}{2 ! c(c+1)} x^{2}+\ldots \\
& =\sum_{n=0}^{+\infty} \frac{a \times \ldots \times(a+n-1) \times b \times \ldots \times(b+n-1)}{n ! c \times \ldots \times(c+n-1)} x^{n}=\sum_{n=0}^{+\infty} \frac{(a)_{n}(b)_{n}}{n !(c)_{n}} x^{n} .
\end{aligned}
$$

In addition, let

$$
\begin{aligned}
\widehat{G}_{I}\left(p_{\mathrm{o}}, p_{\mathrm{n}}, q_{\mathrm{o}}, q_{\mathrm{n}}, t\right) & =1-\frac{e^{-\left(2 p_{\mathrm{o}}+p_{\mathrm{n}}+q_{\mathrm{n}}\right) t}\left(\frac{\left(q_{\mathrm{n}}+p_{\mathrm{n}}\right) e^{\left(q_{\mathrm{n}}+p_{\mathrm{n}}\right) t}}{q_{\mathrm{n}}+p_{\mathrm{n}} e^{\left(q_{\mathrm{n}}+p_{\mathrm{n}}\right) t}}\right)^{\left(\frac{q_{\mathrm{n}}-q_{\mathrm{o}}}{q_{\mathrm{n}}}\right)}}{\left(\frac{\mathcal{M}}{\mathcal{M}-m-M}-\frac{q_{\mathrm{o}}\left(1+\frac{q_{\mathrm{n}}}{p_{\mathrm{n}}}\right)\left(1-\frac{q_{\mathrm{o}}}{q_{\mathrm{n}}}\right)}{2 p_{\mathrm{o}}+p_{\mathrm{n}}+q_{\mathrm{n}}} \widehat{F}_{I}\left(p_{\mathrm{o}}, p_{\mathrm{n}}, q_{\mathrm{o}}, q_{\mathrm{n}}, t\right)\right)}, \\
\widehat{H}_{I}\left(p_{\mathrm{o}}, p_{\mathrm{oc}}, p_{\mathrm{n}}, q_{\mathrm{o}}, q_{\mathrm{n}}, t\right) & =\frac{(M-m)\left(q_{\mathrm{n}}+p_{\mathrm{n}}\right) e^{\left(q_{\mathrm{o}}-2 p_{\mathrm{oc}}\right) t}}{q_{\mathrm{n}}+p_{\mathrm{n}} e^{\left(q_{\mathrm{n}}+p_{\mathrm{n}}\right) t}} e^{-q_{\mathrm{o}} \int_{0}^{t} \widehat{G}_{I}\left(p_{\mathrm{o}}, p_{\mathrm{n}}, q_{\mathrm{o}}, q_{\mathrm{n}}, \tau\right) d \tau}
\end{aligned}
$$

Using the definitions (5)-(8), the time evolution of the customer base trajectories for competing firms in this phase of the diffusion process can be described as follows.

Proposition 1. For $t \in\left[0, t_{f}\right]$, the market share functions

$$
\begin{aligned}
m_{1}^{\mathrm{o}}(t) & =\frac{1}{2}\left(\mathcal{M}\left(\widehat{G}_{I}\left(p_{\mathrm{o}}, p_{\mathrm{n}}, q_{\mathrm{o}}, q_{\mathrm{n}}, t\right)-1+\frac{q_{\mathrm{n}}+p_{\mathrm{n}}}{q_{\mathrm{n}}+p_{\mathrm{n}} e^{\left(q_{\mathrm{n}}+p_{\mathrm{n}}\right) t}}\right)+\widehat{H}_{I}\left(p_{\mathrm{o}}, p_{\mathrm{oc}}, p_{\mathrm{n}}, q_{\mathrm{o}}, q_{\mathrm{n}}, t\right)\right) \\
m_{2}^{\mathrm{o}}(t) & =\frac{1}{2}\left(\mathcal{M}\left(\widehat{G}_{I}\left(p_{\mathrm{o}}, p_{\mathrm{n}}, q_{\mathrm{o}}, q_{\mathrm{n}}, t\right)-1+\frac{q_{\mathrm{n}}+p_{\mathrm{n}}}{q_{\mathrm{n}}+p_{\mathrm{n}} e^{\left(q_{n}+p_{\mathrm{n}}\right) t}}\right)-\widehat{H}_{I}\left(p_{\mathrm{o}}, p_{\mathrm{oc}}, p_{\mathrm{n}}, q_{\mathrm{o}}, q_{\mathrm{n}}, t\right)\right) \\
m_{3}^{\mathrm{n}}(t) & =\mathcal{M}\left(1-\frac{q_{\mathrm{n}}+p_{\mathrm{n}}}{q_{\mathrm{n}}+p_{\mathrm{n}} e^{\left(q_{\mathrm{n}}+p_{\mathrm{n}}\right) t}}\right)
\end{aligned}
$$

solve the competitive diffusion dynamics equations (1)-(3) subject to the initial conditions $m_{1}^{\circ}(0)=$ $M, m_{2}^{\circ}(0)=m$, and $m_{3}^{\mathrm{n}}(0)=0$. 
Proposition 1 states that in a setting where the new entrant is the only provider of the new service model, the time evolution of its customer base is still described by the Bass-like dynamics. However, the corresponding dynamics for the incumbent firms may be substantially different from the one predicted by the monopolistic Bass analysis. The expressions of (9) take a simpler form in two special settings. In the first setting, there is no "word-of-mouth" effect in the old service model dynamics, namely, $q_{\mathrm{o}}=0$. In the second setting, diffusion dynamics proceed without market expansion $(\mathcal{M}=M+m)$ or customer churn $\left(p_{\text {oc }}=0\right)$.

COROLlary 1.1. If $q_{\mathrm{o}}=0$, the customer base trajectories for the competing firms for any $t \in$ $\left[0, t_{f}\right]$ can be expressed as

$$
\begin{aligned}
& m_{1}^{\mathrm{o}}(t)=\frac{1}{2}\left(\frac{\left(q_{\mathrm{n}}+p_{\mathrm{n}}\right)\left(\mathcal{M}-e^{-2 p_{\mathrm{o}} t}(\mathcal{M}-m-M)+(M-m) e^{-2 p_{\mathrm{oc}} t}\right)}{q_{\mathrm{n}}+p_{\mathrm{n}} e^{\left(q_{\mathrm{n}}+p_{\mathrm{n}}\right) t}}\right) \\
& m_{2}^{\mathrm{o}}(t)=\frac{1}{2}\left(\frac{\left(q_{\mathrm{n}}+p_{\mathrm{n}}\right)\left(\mathcal{M}-e^{-2 p_{\mathrm{o}} t}(\mathcal{M}-m-M)-(M-m) e^{-2 p_{\mathrm{oc}} t}\right)}{q_{\mathrm{n}}+p_{\mathrm{n}} e^{\left(q_{\mathrm{n}}+p_{\mathrm{n}}\right) t}}\right) \\
& m_{3}^{\mathrm{n}}(t)=\mathcal{M}\left(1-\frac{q_{\mathrm{n}}+p_{\mathrm{n}}}{q_{\mathrm{n}}+p_{\mathrm{n}} e^{\left(q_{\mathrm{n}}+p_{\mathrm{n}}\right) t}}\right)
\end{aligned}
$$

In the second special setting, the introduction of the innovation does not cause an expansion in the overall market potential $(\mathcal{M}=M+m)$, and there is no intra-generational customer churn $\left(p_{\mathrm{oc}}=0\right)$. In this setting the diffusion dynamics are solely governed by customers switching from the old service model to the new one.

Corollary 1.2. For $\mathcal{M}=M+m$ and $p_{\mathrm{oc}}=0$, the customer bases of the competing firms for any $t \in\left[0, t_{f}\right]$ can be expressed as

$m_{1}^{\mathrm{o}}(t)=M\left(\frac{q_{\mathrm{n}}+p_{\mathrm{n}}}{q_{\mathrm{n}}+p_{\mathrm{n}} e^{\left(q_{\mathrm{n}}+p_{\mathrm{n}}\right) t}}\right), m_{2}^{\mathrm{o}}(t)=m\left(\frac{q_{\mathrm{n}}+p_{\mathrm{n}}}{q_{\mathrm{n}}+p_{\mathrm{n}} e^{\left(q_{\mathrm{n}}+p_{\mathrm{n}}\right) t}}\right), m_{3}^{\mathrm{n}}(t)=\mathcal{M}\left(1-\frac{q_{\mathrm{n}}+p_{\mathrm{n}}}{q_{\mathrm{n}}+p_{\mathrm{n}} e^{\left(q_{\mathrm{n}}+p_{\mathrm{n}}\right) t}}\right)$.

\subsection{Phase II: "New Entrant and One Incumbent"}

In order to counteract the detrimental effect of the new business model on their customer base, the incumbents may decide to adopt the new service model. Let $t_{i}, i=1,2$ be the time at which incumbent $i$ starts offering a new service model alongside its old model, and denote min $\left(t_{1}, t_{2}\right)$ as $t_{f}$ and $\max \left(t_{1}, t_{2}\right)$ as $t_{s}$. Consider the period of time $t \in\left[t_{f}, t_{s}\right]$ after the adoption of the new 
service model by the first incumbent, but before adoption by the second incumbent. Without the loss of generality, we consider the situation where the minor incumbent is first to adopt the new model, namely, $t_{f}=t_{2}$ and $t_{s}=t_{1}$. In the time phase between $t_{f}$ and $t_{s}$, two firms (the entrant and the minor incumbent) offer a new service model, and the market base $\mathcal{M}$ is split into five distinct customer groups. As before, $m_{i}^{\circ}(t), i=1,2$ denote the customers who remain with incumbent firms and with the old service model, $m_{i}^{\mathrm{n}}(t), i=2,3$ correspond to the customers who do business with the minor incumbent or with the entrant using the new service model, while $\mathcal{M}-m_{1}^{\mathrm{o}}(t)-m_{2}^{\circ}(t)-m_{2}^{\mathrm{n}}(t)-m_{3}^{\mathrm{n}}(t)$ corresponds to the group of customers who have not yet adopted any service model. The competitive diffusion dynamics in this phase are described by

$$
\begin{aligned}
\frac{d m_{1}^{o}}{d t}= & \left(p_{\mathrm{o}}+\frac{q_{\mathrm{o}}}{\mathcal{M}} m_{1}^{\mathrm{o}}\right)\left(\mathcal{M}-m_{1}^{\mathrm{o}}-m_{2}^{\mathrm{o}}-m_{2}^{\mathrm{n}}-m_{3}^{\mathrm{n}}\right)+p_{\mathrm{oc}} m_{2}^{\mathrm{o}} \\
& -\left(p_{\mathrm{oc}}+2 p_{\mathrm{n}}+\frac{Q_{\mathrm{n}}}{\mathcal{M}} m_{2}^{\mathrm{n}}+\frac{q_{\mathrm{n}}}{\mathcal{M}} m_{3}^{\mathrm{n}}\right) m_{1}^{\mathrm{o}}, \\
\frac{d m_{2}^{o}}{d t}= & \left(p_{\mathrm{o}}+\frac{q_{\mathrm{o}}}{\mathcal{M}} m_{2}^{\mathrm{o}}\right)\left(\mathcal{M}-m_{1}^{\mathrm{o}}-m_{2}^{\mathrm{o}}-m_{2}^{\mathrm{n}}-m_{3}^{\mathrm{n}}\right)+p_{\mathrm{oc}} m_{1}^{\mathrm{o}} \\
& -\left(p_{\mathrm{oc}}+2 p_{\mathrm{n}}+\frac{Q_{\mathrm{n}}}{\mathcal{M}} m_{2}^{\mathrm{n}}+\frac{q_{\mathrm{n}}}{\mathcal{M}} m_{3}^{\mathrm{n}}\right) m_{2}^{\mathrm{o}}, \\
\frac{d m_{2}^{n}}{d t}= & \left(p_{\mathrm{n}}+\frac{Q_{\mathrm{n}}}{\mathcal{M}} m_{2}^{\mathrm{n}}\right)\left(\mathcal{M}-m_{2}^{\mathrm{n}}-m_{3}^{\mathrm{n}}\right)+p_{\mathrm{nc}} m_{3}^{\mathrm{n}}-p_{\mathrm{nc}} m_{2}^{\mathrm{n}}, \\
\frac{d m_{3}^{n}}{d t}= & \left(p_{\mathrm{n}}+\frac{q_{\mathrm{n}}}{\mathcal{M}} m_{3}^{\mathrm{n}}\right)\left(\mathcal{M}-m_{2}^{\mathrm{n}}-m_{3}^{\mathrm{n}}\right)+p_{\mathrm{nc}} m_{2}^{\mathrm{n}}-p_{\mathrm{nc}} m_{3}^{\mathrm{n}},
\end{aligned}
$$

with the initial conditions

$$
\begin{aligned}
& m_{1}^{\mathrm{o}}\left(t_{f}\right)=\frac{1}{2}\left(\begin{array}{c}
\mathcal{M}\left(\widehat{G}_{I}\left(p_{\mathrm{o}}, p_{\mathrm{n}}, q_{\mathrm{o}}, q_{\mathrm{n}}, t_{f}\right)-1+\frac{q_{\mathrm{n}}+p_{\mathrm{n}}}{q_{\mathrm{n}}+p_{\mathrm{n}} e^{\left(q_{\mathrm{n}}+p_{\mathrm{n}}\right) t_{f}}}\right) \\
+\widehat{H}_{I}\left(p_{\mathrm{o}}, p_{\mathrm{oc}}, p_{\mathrm{n}}, q_{\mathrm{o}}, q_{\mathrm{n}}, t_{f}\right)
\end{array}\right), \\
& m_{2}^{\mathrm{o}}\left(t_{f}\right)=\frac{1}{2}\left(\begin{array}{c}
\mathcal{M}\left(\widehat{G}_{I}\left(p_{\mathrm{o}}, p_{\mathrm{n}}, q_{\mathrm{o}}, q_{\mathrm{n}}, t_{f}\right)-1+\frac{q_{\mathrm{n}}+p_{\mathrm{n}}}{q_{\mathrm{n}}+p_{\mathrm{n}} e^{\left(q_{\mathrm{n}}+p_{\mathrm{n}}\right) t_{f}}}\right) \\
-\widehat{H}_{I}\left(p_{\mathrm{o}}, p_{\mathrm{oc}}, p_{\mathrm{n}}, q_{\mathrm{o}}, q_{\mathrm{n}}, t_{f}\right)
\end{array}\right), \\
& m_{2}^{\mathrm{n}}\left(t_{f}\right)=0, m_{3}^{\mathrm{n}}\left(t_{f}\right)=\mathcal{M}\left(1-\frac{q_{\mathrm{n}}+p_{\mathrm{n}}}{q_{\mathrm{n}}+p_{\mathrm{n}} e^{\left(q_{\mathrm{n}}+p_{\mathrm{n}}\right) t_{f}}}\right),
\end{aligned}
$$

reflecting the results of Proposition 1.

Equations (12) and (13) describe the customer base of the incumbents' old business models, $m_{1}^{o}$ and $m_{2}^{o}$, which evolve via innovation and imitation by non-adopters ( $p_{\mathrm{o}}$ and $q_{\mathrm{o}}$ terms), intragenerational churn ( $p_{\text {oc }}$ terms) and adoption of the new service model $\left(p_{\mathrm{n}}, q_{\mathrm{n}}\right.$, and $Q_{\mathrm{n}}$ terms). In 
this diffusion phase, we allow the imitation terms for the new service model to have an asymmetric form. In particular, the probability of adopting the new model depends on whether the imitation contact occurred with the incumbent or the entrant firm's customer. In numerous settings, incumbents enjoy a stronger imitation process due to brand awareness. We model this by using different parameters to describe the imitation of the incumbent's new service model $\left(Q_{\mathrm{n}}\right)$, and the entrant's service model $\left(q_{\mathrm{n}}\right.$ with $\left.q_{\mathrm{n}} \leq Q_{\mathrm{n}}\right)$. Strong brand awareness results in shorter take-off periods for the incumbent's customer base, as suggested by equation (14). Modeling the brand awareness effect provides an additional layer of realism to the model, while increasing its analytical complexity. Finally, equation (15) describes the dynamics of the new entrant's customer base, evolving through innovation, imitation and churn. Note that in (14)-(15) the parameter $p_{\text {nc }}$ describes the inter-firm churn process for customers that use the new service model.

The diffusion dynamics (12)-(15), while providing a detailed description of a competitive diffusion setting, yield closed-form solutions for the customer base trajectories in two special settings described below.

2.2.1. Ballooning Market with Symmetric Brand Effect In this section we consider a setting allowing market expansion $(\mathcal{M} \geq M+m)$ and intra-generational churn $\left(p_{\mathrm{oc}}, p_{\mathrm{nc}}>0\right)$. In addition, we assume that the imitation process in the adoption of the new service model is symmetric. In other words, we assume that the incumbent's new service model adoption does not enjoy a brand awareness advantage over the new entrant's one, so that $Q_{\mathrm{n}}=q_{\mathrm{n}}$. This assumption is along the lines of the brand communication model of Krishnan et al. (2000), whereby cross-brand and intra-brand influences are equal.

For a given set of diffusion parameters $p_{o}, q_{o}, p_{n}$, and $q_{n}$, define

$$
\begin{aligned}
\alpha= & \frac{\left(2 p_{\mathrm{n}}+q_{\mathrm{n}}\right)\left(q_{\mathrm{n}}+p_{\mathrm{n}} e^{\left(p_{n}+q_{n}\right) t_{f}}\right)}{p_{\mathrm{n}}+q_{\mathrm{n}}}-q_{\mathrm{n}}, \\
\widehat{F}_{I I}\left(p_{\mathrm{o}}, p_{\mathrm{n}}, q_{\mathrm{o}}, q_{\mathrm{n}}, t\right)= & { }_{2} F_{1}\left(1, \frac{2 p_{\mathrm{o}}+2 p_{\mathrm{n}}+q_{\mathrm{n}}}{2 p_{\mathrm{n}}+q_{\mathrm{n}}}+\frac{q_{\mathrm{o}}}{q_{\mathrm{n}}} ; \frac{2\left(p_{\mathrm{o}}+2 p_{\mathrm{n}}+q_{\mathrm{n}}\right)}{2 p_{\mathrm{n}}+q_{\mathrm{n}}} ;-\frac{q_{\mathrm{n}}}{\alpha}\right) \\
& -e^{-\left(2 p_{\mathrm{o}}+2 p_{\mathrm{n}}+q_{\mathrm{n}}\right)\left(t-t_{f}\right)}\left(\frac{\alpha+q_{\mathrm{n}} e^{-\left(2 p_{\mathrm{n}}+q_{\mathrm{n}}\right)\left(t-t_{f}\right)}}{\alpha+q_{\mathrm{n}}}\right)^{\frac{q_{\mathrm{o}}}{q_{\mathrm{n}}}}
\end{aligned}
$$




$$
\times{ }_{2} F_{1}\left(1, \frac{2 p_{\mathrm{o}}+2 p_{\mathrm{n}}+q_{\mathrm{n}}}{2 p_{\mathrm{n}}+q_{\mathrm{n}}}+\frac{q_{\mathrm{o}}}{q_{\mathrm{n}}} ; \frac{2\left(p_{\mathrm{o}}+2 p_{\mathrm{n}}+q_{\mathrm{n}}\right)}{2 p_{\mathrm{n}}+q_{\mathrm{n}}} ;-\frac{q_{\mathrm{n}}}{\alpha} e^{-\left(2 p_{\mathrm{n}}+q_{\mathrm{n}}\right)\left(t-t_{f}\right)}\right) .
$$

In addition, let

$$
\begin{aligned}
& \widehat{G}_{I I}\left(p_{\mathrm{o}}, p_{\mathrm{n}}, q_{\mathrm{o}}, q_{\mathrm{n}}, t\right)=1-\frac{e^{-\left(2 p_{\mathrm{o}}+2 p_{\mathrm{n}}+q_{\mathrm{n}}\right)\left(t-t_{f}\right)}\left(\frac{\left(q_{\mathrm{n}}+\alpha\right) e^{\left(q_{\mathrm{n}}+2 p_{\mathrm{n}}\right)\left(t-t_{f}\right)}}{q_{\mathrm{n}}+\alpha e^{\left(q_{\mathrm{n}}+2 p_{\mathrm{n}}\right)\left(t-t_{f}\right)}}\right)^{\left(\frac{q_{\mathrm{n}}-q_{\mathrm{o}}}{q_{\mathrm{n}}}\right)}}{\left(\frac{1}{1-\widehat{G}_{I}\left(p_{\mathrm{o}}, p_{\mathrm{n}}, q_{\mathrm{o}}, q_{\mathrm{n}}, t_{f}\right)}-\frac{q_{\mathrm{o}}\left(\alpha+q_{\mathrm{n}}\right)}{\alpha\left(2 p_{\mathrm{o}}+2 p_{\mathrm{n}}+q_{\mathrm{n}}\right)} \widehat{F}_{I I}\left(p_{\mathrm{o}}, p_{\mathrm{n}}, q_{\mathrm{o}}, q_{\mathrm{n}}, t\right)\right)}, \\
& \widehat{H}_{I I}\left(p_{\mathrm{o}}, p_{\mathrm{oc}}, p_{\mathrm{n}}, q_{\mathrm{o}}, q_{\mathrm{n}}, t\right)=\frac{\widehat{H}_{I}\left(p_{\mathrm{o}}, p_{\mathrm{oc}}, p_{\mathrm{n}}, q_{\mathrm{o}}, q_{\mathrm{n}}, t_{f}\right)\left(\alpha+q_{\mathrm{n}}\right) e^{\left(q_{\mathrm{o}}-2 p_{\mathrm{oc}}\right)\left(t-t_{f}\right)}}{q_{\mathrm{n}}+\alpha e^{\left(q_{\mathrm{n}}+2 p_{\mathrm{n}}\right)\left(t-t_{f}\right)}} \\
& \times e^{-q_{\mathrm{o}} \int_{t}^{t} \widehat{G}_{I I}\left(p_{\mathrm{o}}, p_{\mathrm{n}}, q_{\mathrm{o}}, q_{\mathrm{n}}, \tau\right) d \tau}, \\
& n(t)=\mathcal{M}\left(1-\frac{2 p_{\mathrm{n}}+q_{\mathrm{n}}}{\alpha e^{\left(2 p_{\mathrm{n}}+q_{\mathrm{n}}\right)\left(t-t_{f}\right)}+q_{\mathrm{n}}}\right), \\
& a(t)=\frac{m_{3}^{\mathrm{n}}\left(t_{f}\right) e^{\left(2\left(p_{\mathrm{n}}-p_{\mathrm{nc}}\right)+q_{\mathrm{n}}\right)\left(t-t_{f}\right)}\left(\alpha+q_{\mathrm{n}}\right)}{\alpha e^{\left(2 p_{\mathrm{n}}+q_{\mathrm{n}}\right)\left(t-t_{f}\right)}+q_{\mathrm{n}}} \\
& =\mathcal{M}\left(\frac{p_{\mathrm{n}}\left(2 p_{\mathrm{n}}+q_{\mathrm{n}}\right) e^{\left(2 p_{\mathrm{n}}-2 p_{\mathrm{nc}}+q_{\mathrm{n}}\right)\left(t-t_{f}\right)}\left(e^{\left(p_{\mathrm{n}}+q_{\mathrm{n}}\right) t_{f}}-1\right)}{\left(\alpha e^{\left(2 p_{\mathrm{n}}+q_{\mathrm{n}}\right)\left(t-t_{f}\right)}+q_{\mathrm{n}}\right)\left(p_{\mathrm{n}}+q_{\mathrm{n}}\right)}\right) .
\end{aligned}
$$

Then, the diffusion dynamics in this phase can be described as follows.

Proposition 2. For any $t \in\left[t_{f}, t_{s}\right]$ the market shares can be expressed as

$$
\begin{aligned}
& m_{1}^{\mathrm{o}}(t)=\frac{\mathcal{M} \widehat{G}_{I I}\left(p_{\mathrm{o}}, p_{\mathrm{n}}, q_{\mathrm{o}}, q_{\mathrm{n}}, t\right)-n(t)+\widehat{H}_{I I}\left(p_{\mathrm{o}}, p_{\mathrm{oc}}, p_{\mathrm{n}}, q_{\mathrm{o}}, q_{\mathrm{n}}, t\right)}{2}, \\
& m_{2}^{\mathrm{o}}(t)=\frac{\mathcal{M} \widehat{G}_{I I}\left(p_{\mathrm{o}}, p_{\mathrm{n}}, q_{\mathrm{o}}, q_{\mathrm{n}}, t\right)-n(t)-\widehat{H}_{I I}\left(p_{\mathrm{o}}, p_{\mathrm{oc}}, p_{\mathrm{n}}, q_{\mathrm{o}}, q_{\mathrm{n}}, t\right)}{2}, \\
& m_{2}^{\mathrm{n}}(t)=\frac{n(t)-a(t)}{2}, m_{3}^{\mathrm{n}}(t)=\frac{n(t)+a(t)}{2} .
\end{aligned}
$$

We can get simpler closed-form expressions for customer base trajectories, considering the case where there is no "word-of-mouth" effect for the old service model.

COROLlary 2.1. If $q_{\mathrm{o}}=0$, the customer base trajectories of the competing firms for any $t \in$ $\left[0, t_{f}\right]$ can be expressed as

$$
\begin{aligned}
& m_{1}^{\mathrm{o}}(t)=\frac{1}{2}\left(\frac{\left(q_{\mathrm{n}}+2 p_{\mathrm{n}}\right)\left(\mathcal{M}-e^{-2 p_{\mathrm{o}} t}(\mathcal{M}-m-M)+(M-m) e^{-2 p_{\mathrm{oc}} t}\right)}{q_{\mathrm{n}}+\alpha e^{\left(q_{\mathrm{n}}+2 p_{\mathrm{n}}\right)\left(t-t_{f}\right)}}\right) \\
& m_{2}^{\mathrm{o}}(t)=\frac{1}{2}\left(\frac{\left(q_{\mathrm{n}}+2 p_{\mathrm{n}}\right)\left(\mathcal{M}-e^{-2 p_{\mathrm{o}} t}(\mathcal{M}-m-M)-(M-m) e^{-2 p_{\mathrm{oc}} t}\right)}{q_{\mathrm{n}}+\alpha e^{\left(q_{\mathrm{n}}+2 p_{\mathrm{n}}\right)\left(t-t_{f}\right)}}\right) \\
& m_{2}^{\mathrm{n}}(t)=\frac{n(t)-a(t)}{2}, m_{3}^{\mathrm{n}}(t)=\frac{n(t)+a(t)}{2} .
\end{aligned}
$$


2.2.2. Mature Market with Asymmetric Brand Effect As in the previous phase, we restrict the focus to a setting where a) the new service model does not cause an expansion in the overall market potential $(\mathcal{M}=M+m)$, and b) there is no intra-generational customer churn $\left(p_{\mathrm{oc}}=p_{\mathrm{nc}}=0\right)$. This assumption is similar to the brand communication model of Kalish et al. (1995), whereby the influence of communication is only within brand. However, we allow the incumbents to enjoy a brand awareness advantage in customer imitation by letting $Q_{\mathrm{n}}>q_{\mathrm{n}}$. For the convenience of future analysis, we introduce the following notation corresponding to relative (percentage) customer bases:

$$
o_{i}(t)=\frac{m_{i}^{\mathrm{o}}(t)}{\mathcal{M}}, i=1,2, n_{i}(t)=\frac{m_{i}^{\mathrm{n}}(t)}{\mathcal{M}}, i=1,2,3,
$$

and $\theta=\frac{M}{\mathcal{M}}$. Also, for $Q_{\mathrm{n}}>0$, let

$$
S(x, z)=\left\{\begin{array}{c}
\frac{p_{\mathrm{n}}}{Q_{\mathrm{n}}}\left(\left(\frac{p_{\mathrm{n}}+q_{\mathrm{n}} x}{p_{\mathrm{n}}+q_{\mathrm{n}} z}\right)^{\frac{Q_{\mathrm{n}}}{q_{\mathrm{n}}}}-1\right), \\
\frac{q_{\mathrm{n}}>0,}{Q_{\mathrm{n}}}\left(e^{\frac{Q_{\mathrm{n}}(x-z)}{p_{\mathrm{n}}}}-1\right), \quad q_{\mathrm{n}}=0 .
\end{array}\right.
$$

Proposition 3. If the introduction of the new service model does not expand the overall market potential $(\mathcal{M}=M+m)$, and there is no intra-generational customer churn $\left(p_{\mathrm{oc}}=p_{\mathrm{nc}}=0\right)$, the percentage customer base trajectories of the competing firms for any $t \in\left[t_{f}, t_{s}\right]$ can be expressed as

$$
\begin{aligned}
& t-t_{f}=\int_{n_{3}\left(t_{f}\right)}^{n_{3}(t)} \frac{d y}{\left(p_{\mathrm{n}}+q_{\mathrm{n}} y\right)\left(1-y-S\left(y, n_{3}\left(t_{f}\right)\right)\right)}, \\
& o_{1}(t)=\theta\left(1-n_{3}(t)-S\left(n_{3}(t), n_{3}\left(t_{f}\right)\right)\right), \\
& o_{2}(t)=(1-\theta)\left(1-n_{3}(t)-S\left(n_{3}(t), n_{3}\left(t_{f}\right)\right)\right), \\
& n_{2}(t)=S\left(n_{3}(t), n_{3}\left(t_{f}\right)\right) .
\end{aligned}
$$

Proposition 3 expresses the customer bases of present firm/service model combinations at any point in time through the value of an easy-to-evaluate integral. The expressions of (29) can be further simplified in the case when the word-of-mouth effects of the new service model are firmindependent, so that $q_{\mathrm{n}}=Q_{\mathrm{n}}$. 
COROLLARY 3.1. When the introduction of the new service model does not expand the overall market potential $(\mathcal{M}=M+m)$, there is no intra-generational customer churn $\left(p_{\mathrm{oc}}=p_{\mathrm{nc}}=0\right)$, and imitation occurs through symmetric terms $\left(q_{\mathrm{n}}=Q_{\mathrm{n}}>0\right)$, the customer bases of the competing firms for any $t \in\left[t_{f}, t_{s}\right]$ can be expressed as

$$
\begin{aligned}
n_{3}(t)= & \frac{p_{\mathrm{n}}\left(1-\frac{p_{\mathrm{n}}}{q_{\mathrm{n}}}\right)+\left(p_{\mathrm{n}}+q_{\mathrm{n}}\right) n_{3}\left(t_{f}\right)}{2 p_{\mathrm{n}}+q_{\mathrm{n}} n_{3}\left(t_{f}\right)} \\
& -\frac{\left(p_{\mathrm{n}}\left(q_{\mathrm{n}}+p_{\mathrm{n}}\right)+q_{\mathrm{n}}\left(2 p_{\mathrm{n}}+q_{\mathrm{n}}\right) n_{3}\left(t_{f}\right)\right)\left(p_{\mathrm{n}}\left(1-\frac{p_{\mathrm{n}}}{q_{\mathrm{n}}}\right)-n_{3}\left(t_{f}\right)\left(p_{\mathrm{n}}-q_{\mathrm{n}}+q_{\mathrm{n}} n_{3}\left(t_{f}\right)\right)\right)}{\left(\left(p_{\mathrm{n}}+q_{\mathrm{n}} n_{3}\left(t_{f}\right)\right)\left(2 p_{\mathrm{n}}+q_{\mathrm{n}} n_{3}\left(t_{f}\right)\right)^{2} e^{\frac{\left(p_{\mathrm{n}}\left(2 p_{\mathrm{n}}+q_{\mathrm{n}} n_{3}\left(t_{f}\right)\right)+p_{\mathrm{n}}\left(q_{\mathrm{n}}-p_{\mathrm{n}}\right)+q_{\mathrm{n}}\left(p_{\mathrm{n}}+q_{\mathrm{n}}\right) n_{3}\left(t_{f}\right)\right)\left(t-t_{f}\right)}{\left(p_{\mathrm{n}}+q_{\mathrm{n}} n_{3}\left(t_{f}\right)\right)}}+1\right)}, \\
o_{1}(t)= & \theta\left(1-n_{3}(t)-p_{\mathrm{n}}\left(\frac{n_{3}(t)-n_{3}\left(t_{f}\right)}{p_{\mathrm{n}}+q_{\mathrm{n}} n_{3}\left(t_{f}\right)}\right)\right), o_{2}(t)=(1-\theta)\left(1-n_{3}(t)-p_{\mathrm{n}}\left(\frac{n_{3}(t)-n_{3}\left(t_{f}\right)}{p_{\mathrm{n}}+q_{\mathrm{n}} n_{3}\left(t_{f}\right)}\right)\right), \\
n_{2}(t)= & p_{\mathrm{n}}\left(\frac{n_{3}(t)-n_{3}\left(t_{f}\right)}{p_{\mathrm{n}}+q_{\mathrm{n}} n_{3}\left(t_{f}\right)}\right) .
\end{aligned}
$$

\subsection{Phase III: "New Entrant and Two Incumbents"}

For any time $t>t_{s}$, in addition to the customer groups described in the previous section, there appears $m_{1}^{\mathrm{n}}(t)$, the group of customers who adopt the new service model through the incumbent firm 1 . The diffusion dynamics are now described by the following set of differential equations:

$$
\begin{aligned}
\frac{d m_{1}^{\mathrm{o}}}{d t}= & \left(p_{\mathrm{o}}+\frac{q_{\mathrm{o}}}{\mathcal{M}} m_{1}^{\mathrm{o}}\right)\left(\mathcal{M}-m_{1}^{\mathrm{o}}-m_{2}^{\mathrm{o}}-m_{1}^{\mathrm{n}}-m_{2}^{\mathrm{n}}-m_{3}^{\mathrm{n}}\right)+p_{\mathrm{oc}} m_{2}^{\mathrm{o}} \\
& -\left(p_{\mathrm{oc}}+3 p_{\mathrm{n}}+\frac{Q_{\mathrm{n}}}{\mathcal{M}}\left(m_{1}^{\mathrm{n}}+m_{2}^{\mathrm{n}}\right)+\frac{q_{\mathrm{n}}}{\mathcal{M}} m_{3}^{\mathrm{n}}\right) m_{1}^{\mathrm{o}}, \\
\frac{d m_{2}^{\mathrm{o}}}{d t}= & \left(p_{\mathrm{o}}+\frac{q_{\mathrm{o}}}{\mathcal{M}} m_{2}^{\mathrm{o}}\right)\left(\mathcal{M}-m_{1}^{\mathrm{o}}-m_{2}^{\mathrm{o}}-m_{1}^{\mathrm{n}}-m_{2}^{\mathrm{n}}-m_{3}^{\mathrm{n}}\right)+p_{\mathrm{oc}} m_{1}^{\mathrm{o}} \\
& -\left(p_{\mathrm{oc}}+3 p_{\mathrm{n}}+\frac{Q_{\mathrm{n}}}{\mathcal{M}}\left(m_{1}^{\mathrm{n}}+m_{2}^{\mathrm{n}}\right)+\frac{q_{\mathrm{n}}}{\mathcal{M}} m_{3}^{\mathrm{n}}\right) m_{2}^{\mathrm{o}}, \\
\frac{d m_{1}^{\mathrm{n}}}{d t}= & \left(p_{\mathrm{n}}+\frac{Q_{\mathrm{n}}}{\mathcal{M}} m_{1}^{\mathrm{n}}\right)\left(\mathcal{M}-m_{1}^{\mathrm{n}}-m_{2}^{\mathrm{n}}-m_{3}^{\mathrm{n}}\right)+p_{\mathrm{nc}}\left(m_{2}^{\mathrm{n}}+m_{3}^{\mathrm{n}}\right)-2 p_{\mathrm{nc}} m_{1}^{\mathrm{n}}, \\
\frac{d m_{2}^{\mathrm{n}}}{d t}= & \left(p_{\mathrm{n}}+\frac{Q_{\mathrm{n}}}{\mathcal{M}} m_{2}^{\mathrm{n}}\right)\left(\mathcal{M}-m_{1}^{\mathrm{n}}-m_{2}^{\mathrm{n}}-m_{3}^{\mathrm{n}}\right)+p_{\mathrm{nc}}\left(m_{1}^{\mathrm{n}}+m_{3}^{\mathrm{n}}\right)-2 p_{\mathrm{nc}} m_{2}^{\mathrm{n}}, \\
\frac{d m_{3}^{\mathrm{n}}}{d t}= & \left(p_{\mathrm{n}}+\frac{q_{\mathrm{n}}}{\mathcal{M}} m_{3}^{\mathrm{n}}\right)\left(\mathcal{M}-m_{1}^{\mathrm{n}}-m_{2}^{\mathrm{n}}-m_{3}^{\mathrm{n}}\right)+p_{\mathrm{nc}}\left(m_{1}^{\mathrm{n}}+m_{2}^{\mathrm{n}}\right)-2 p_{\mathrm{nc}} m_{3}^{\mathrm{n}}
\end{aligned}
$$

augmented by the initial conditions following from the solution of the previous phase. For example, the initial conditions in the case of symmetric imitation $\left(Q_{\mathrm{n}}=q_{\mathrm{n}}\right)(31)-(35)$ are

$$
m_{1}^{o}\left(t_{s}\right)=\frac{\mathcal{M} \widehat{G}_{I I}\left(p_{\mathrm{o}}, p_{\mathrm{n}}, q_{\mathrm{o}}, q_{\mathrm{n}}, t_{s}\right)-n(t)+\widehat{H}_{I I}\left(p_{\mathrm{o}}, p_{\mathrm{oc}}, p_{\mathrm{n}}, q_{\mathrm{o}}, q_{\mathrm{n}}, t_{s}\right)}{2},
$$




$$
\begin{aligned}
& m_{2}^{o}\left(t_{s}\right)=\frac{\mathcal{M} \widehat{G}_{I I}\left(p_{\mathrm{o}}, p_{\mathrm{n}}, q_{\mathrm{o}}, q_{\mathrm{n}}, t_{s}\right)-n(t)-\widehat{H}_{I I}\left(p_{\mathrm{o}}, p_{\mathrm{oc}}, p_{\mathrm{n}}, q_{\mathrm{o}}, q_{\mathrm{n}}, t_{s}\right)}{2} \\
& m_{1}^{n}\left(t_{s}\right)=0, m_{2}^{n}\left(t_{s}\right)=\frac{n\left(t_{s}\right)-a\left(t_{s}\right)}{2}, m_{3}^{n}\left(t_{s}\right)=\frac{n\left(t_{s}\right)+a\left(t_{s}\right)}{2} .
\end{aligned}
$$

2.3.1. Ballooning Market with Symmetric Brand Effect As in the previous phases, we first consider the setting allowing market expansion $(\mathcal{M} \geq M+m)$ and intra-generational churn $\left(p_{\mathrm{oc}}, p_{\mathrm{nc}}>0\right)$, while restricting ourselves to symmetric word-of-mouth effects for the new service model $\left(Q_{\mathrm{n}}=q_{\mathrm{n}}\right)$. For a given set of diffusion parameters $p_{o}, q_{o}, p_{n}$, and $q_{n}$, define

$$
\begin{gathered}
\lambda=\frac{\left(3 p_{\mathrm{n}}+q_{\mathrm{n}}\right)\left(q_{\mathrm{n}}+\alpha e^{\left(2 p_{n}+q_{n}\right)\left(t_{s}-t_{f}\right)}\right)}{2 p_{\mathrm{n}}+q_{\mathrm{n}}}-q_{\mathrm{n}} \\
\widehat{F}_{I I I}\left(p_{\mathrm{o}}, p_{\mathrm{n}}, q_{\mathrm{o}}, q_{\mathrm{n}}, t\right)={ }_{2} F_{1}\left(1, \frac{2 p_{\mathrm{o}}+3 p_{\mathrm{n}}+q_{\mathrm{n}}}{3 p_{\mathrm{n}}+q_{\mathrm{n}}}+\frac{q_{\mathrm{o}}}{q_{\mathrm{n}}} ; \frac{2\left(p_{\mathrm{o}}+3 p_{\mathrm{n}}+q_{\mathrm{n}}\right)}{3 p_{\mathrm{n}}+q_{\mathrm{n}}} ;-\frac{q_{\mathrm{n}}}{\lambda}\right) \\
-e^{-\left(2 p_{\mathrm{o}}+3 p_{\mathrm{n}}+q_{\mathrm{n}}\right)\left(t-t_{\mathrm{s}}\right)}\left(\frac{\lambda+q_{\mathrm{n}} e^{-\left(3 p_{\mathrm{n}}+q_{\mathrm{n}}\right)\left(t-t_{s}\right)}}{\lambda+q_{\mathrm{n}}}\right)^{\frac{q_{\mathrm{o}}}{q_{\mathrm{n}}}} \\
\times{ }_{2} F_{1}\left(1, \frac{2 p_{\mathrm{o}}+3 p_{\mathrm{n}}+q_{\mathrm{n}}}{3 p_{\mathrm{n}}+q_{\mathrm{n}}}+\frac{q_{\mathrm{o}}}{q_{\mathrm{n}}} ; \frac{2\left(p_{\mathrm{o}}+3 p_{\mathrm{n}}+q_{\mathrm{n}}\right)}{3 p_{\mathrm{n}}+q_{\mathrm{n}}} ;-\frac{q_{\mathrm{n}}}{\lambda} e^{-\left(3 p_{\mathrm{n}}+q_{\mathrm{n}}\right)\left(t-t_{s}\right)}\right),
\end{gathered}
$$

In addition, let

$$
\begin{aligned}
& \widehat{G}_{I I I}\left(p_{\mathrm{o}}, p_{\mathrm{n}}, q_{\mathrm{o}}, q_{\mathrm{n}}, t\right)=1-\frac{e^{-\left(2 p_{\mathrm{o}}+3 p_{\mathrm{n}}+q_{\mathrm{n}}\right)\left(t-t_{s}\right)}\left(\frac{\left(q_{\mathrm{n}}+\lambda\right) e^{\left(q_{\mathrm{n}}+3 p_{\mathrm{n}}\right)\left(t-t_{s}\right)}}{q_{\mathrm{n}}+\lambda e^{\left(q_{\mathrm{n}}+3 p_{\mathrm{n}}\right)\left(t-t_{s}\right)}}\right)^{\left(\frac{q_{\mathrm{n}}-q_{\mathrm{o}}}{q_{\mathrm{n}}}\right)}}{\left(\frac{1}{1-\widehat{G}_{I I}\left(p_{\mathrm{o}}, p_{\mathrm{n}}, q_{\mathrm{o}}, q_{\mathrm{n}}, t_{s}\right)}-\frac{q_{\mathrm{o}}\left(\lambda+q_{\mathrm{n}}\right)}{\lambda\left(2 p_{\mathrm{o}}+3 p_{\mathrm{n}}+q_{\mathrm{n}}\right)} \widehat{F}_{I I I}\left(p_{\mathrm{o}}, p_{\mathrm{n}}, q_{\mathrm{o}}, q_{\mathrm{n}}, t\right)\right)}, \\
& \widehat{H}_{I I I}\left(p_{\mathrm{o}}, p_{\mathrm{oc}}, p_{\mathrm{n}}, q_{\mathrm{o}}, q_{\mathrm{n}}, t\right)=\frac{\widehat{H}_{I I}\left(p_{\mathrm{o}}, p_{\mathrm{oc}}, p_{\mathrm{n}}, q_{\mathrm{o}}, q_{\mathrm{n}}, t_{s}\right)\left(\lambda+q_{\mathrm{n}}\right) e^{\left(q_{\mathrm{o}}-2 p_{\mathrm{oc}}\right)\left(t-t_{s}\right)}}{q_{\mathrm{n}}+\lambda e^{\left(q_{\mathrm{n}}+3 p_{\mathrm{n}}\right)\left(t-t_{s}\right)}} \\
& \times e^{-q_{\mathrm{o}} \int_{t_{s}}^{t} \widehat{G}_{I I I}\left(p_{\mathrm{o}}, p_{\mathrm{n}}, q_{\mathrm{o}}, q_{\mathrm{n}}, \tau\right) d \tau}, \\
& \widehat{n}(t)=\mathcal{M}\left(1-\frac{3 p_{\mathrm{n}}+q_{\mathrm{n}}}{\lambda e^{\left(3 p_{\mathrm{n}}+q_{\mathrm{n}}\right)\left(t-t_{s}\right)}+q_{\mathrm{n}}}\right), \\
& \widehat{a}(t)=\frac{a\left(t_{s}\right) e^{\left(3\left(p_{\mathrm{n}}-p_{\mathrm{nc}}\right)+q_{\mathrm{n}}\right)\left(t-t_{s}\right)}\left(\lambda+q_{\mathrm{n}}\right)}{\lambda e^{\left(3 p_{\mathrm{n}}+q_{\mathrm{n}}\right)\left(t-t_{s}\right)}+q_{\mathrm{n}}}, \widehat{b}(t)=\frac{m_{2}^{\mathrm{n}}\left(t_{s}\right) e^{\left(3\left(p_{\mathrm{n}}-p_{\mathrm{nc}}\right)+q_{\mathrm{n}}\right)\left(t-t_{s}\right)}\left(\lambda+q_{\mathrm{n}}\right)}{\lambda e^{\left(3 p_{\mathrm{n}}+q_{\mathrm{n}}\right)\left(t-t_{s}\right)}+q_{\mathrm{n}}} .
\end{aligned}
$$

The diffusion dynamics in this phase can be described as follows.

Proposition 4. For $t>t_{s}$, the competitive diffusion dynamics can be expressed as

$$
\begin{aligned}
& m_{1}^{o}(t)=\frac{\mathcal{M} \widehat{G}_{I I I}\left(p_{\mathrm{o}}, p_{\mathrm{n}}, q_{\mathrm{o}}, q_{\mathrm{n}}, t\right)-\widehat{n}(t)+\widehat{H}_{I I I}\left(p_{\mathrm{o}}, p_{\mathrm{oc}}, p_{\mathrm{n}}, q_{\mathrm{o}}, q_{\mathrm{n}}, t\right)}{2}, \\
& m_{2}^{o}(t)=\frac{\mathcal{M} \widehat{G}_{I I I}\left(p_{\mathrm{o}}, p_{\mathrm{n}}, q_{\mathrm{o}}, q_{\mathrm{n}}, t\right)-\widehat{n}(t)-\widehat{H}_{I I I}\left(p_{\mathrm{o}}, p_{\mathrm{oc}}, p_{\mathrm{n}}, q_{\mathrm{o}}, q_{\mathrm{n}}, t\right)}{2}, \\
& m_{1}^{n}(t)=\frac{\widehat{n}(t)-2 \widehat{b}(t)-\widehat{a}(t)}{3}, m_{2}^{n}(t)=\frac{n(t)+\widehat{b}(t)-\widehat{a}(t)}{3}, m_{3}^{n}(t)=\frac{n(t)+\widehat{b}(t)+2 \widehat{a}(t)}{3} .
\end{aligned}
$$


Similar to the dynamics in the previous phase, generalized expressions of (45) convert to simpler closed-form when there is no "word-of-mouth" effect for the old service model.

COROLlaRY 4.1. When there is no word-of-mouth effect for the old service model $\left(q_{\mathrm{o}}=0\right)$, the customer bases of the competing firms for $t>t_{s}$, can be expressed as

$$
\begin{aligned}
m_{1}^{\mathrm{o}}(t) & =\frac{1}{2}\left(\frac{\left(q_{\mathrm{n}}+3 p_{\mathrm{n}}\right)\left(\mathcal{M}-e^{-2 p_{\mathrm{o}} t}(\mathcal{M}-m-M)+(M-m) e^{-2 p_{\mathrm{oc}} t}\right)}{q_{\mathrm{n}}+\lambda e^{\left(q_{\mathrm{n}}+3 p_{\mathrm{n}}\right)\left(t-t_{s}\right)}}\right) \\
m_{2}^{\mathrm{o}}(t) & =\frac{1}{2}\left(\frac{\left(q_{\mathrm{n}}+3 p_{\mathrm{n}}\right)\left(\mathcal{M}-e^{-2 p_{\mathrm{o}} t}(\mathcal{M}-m-M)-(M-m) e^{-2 p_{\mathrm{oc}} t}\right)}{q_{\mathrm{n}}+\lambda e^{\left(q_{\mathrm{n}}+3 p_{\mathrm{n}}\right)\left(t-t_{s}\right)}}\right) \\
m_{1}^{n}(t) & =\frac{\widehat{n}(t)-2 \widehat{b}(t)-\widehat{a}(t)}{3}, m_{2}^{\mathrm{n}}(t)=\frac{\widehat{n}(t)+\widehat{b}(t)-\widehat{a}(t)}{3}, m_{3}^{\mathrm{n}}(t)=\frac{\widehat{n}(t)+\widehat{b}(t)+2 \widehat{a}(t)}{3} .
\end{aligned}
$$

2.3.2. Mature Market with Asymmetric Brand Effect Once again, we focus on the adoption of the new service model, assuming that the new model does not expand the potential market $(\mathcal{M}=M+m)$ and there is no intra-generational customer churn $\left(p_{\mathrm{oc}}=p_{\mathrm{nc}}=0\right)$. Nevertheless, we allow for asymmetric imitation $\left(Q_{\mathrm{n}}>q_{\mathrm{n}}\right)$ to reflect the incumbents' potential brand awareness advantage.

Proposition 5. When the introduction of the new service model does not expand the overall market potential $(\mathcal{M}=M+m)$, and there is no intra-generational customer churn $\left(p_{\mathrm{oc}}=p_{\mathrm{nc}}=0\right)$, the percentage customer bases of the competing firms for $t \geq t_{s}$, can be expressed as

$$
\begin{aligned}
t-t_{s} & =\int_{n_{3}\left(t_{s}\right)}^{n_{3}(t)} \frac{d y}{\left(p_{\mathrm{n}}+q_{\mathrm{n}} y\right)\left(1-S\left(n_{3}\left(t_{s}\right), n_{3}\left(t_{f}\right)\right)-y-S\left(y, n_{3}\left(t_{s}\right)\right)\left(2+\frac{Q_{\mathrm{n}}}{p_{\mathrm{n}}} S\left(n_{3}\left(t_{s}\right), n_{3}\left(t_{f}\right)\right)\right)\right)} \\
o_{1}(t) & =\theta\left(1-n_{3}(t)-S\left(n_{3}(t), n_{3}\left(t_{s}\right)\right)\left(2+\frac{Q_{\mathrm{n}}}{p_{\mathrm{n}}} S\left(n_{3}\left(t_{s}\right), n_{3}\left(t_{f}\right)\right)\right)-S\left(n_{3}\left(t_{s}\right), n_{3}\left(t_{f}\right)\right)\right), \\
o_{2}(t) & =(1-\theta)\left(1-n_{3}(t)-S\left(n_{3}(t), n_{3}\left(t_{s}\right)\right)\left(2+\frac{Q_{\mathrm{n}}}{p_{\mathrm{n}}} S\left(n_{3}\left(t_{s}\right), n_{3}\left(t_{f}\right)\right)\right)-S\left(n_{3}\left(t_{s}\right), n_{3}\left(t_{f}\right)\right)\right) \\
n_{1}(t) & =S\left(n_{3}(t), n_{3}\left(t_{s}\right)\right), n_{2}(t)=S\left(n_{3}(t), n_{3}\left(t_{s}\right)\right)\left(1+\frac{Q_{\mathrm{n}}}{p_{\mathrm{n}}} S\left(n_{3}\left(t_{s}\right), n_{3}\left(t_{f}\right)\right)\right)+S\left(n_{3}\left(t_{s}\right), n_{3}\left(t_{f}\right)\right) .
\end{aligned}
$$

As in the previous phase, expressions in (47) convert to the closed-form in the environment where the imitation coefficients do not depend on the firm through which the new service model was adopted: 
COROLlary 5.1. When the introduction of the new service model does not cause an expansion in the overall market potential $(\mathcal{M}=M+m)$, there is no intra-generational churn $\left(p_{\mathrm{oc}}=p_{\mathrm{nc}}=0\right)$, and imitation occurs through symmetric terms $\left(q_{\mathrm{n}}=Q_{\mathrm{n}}>0\right)$, the customer base trajectories for the competing firms for $t \geq t_{s}$, can be expressed as

$n_{3}(t)=\frac{n_{3}\left(t_{s}\right)\left(q_{\mathrm{n}}+3 p_{\mathrm{n}}\right)+p_{\mathrm{n}}\left(1-e^{-\left(q_{\mathrm{n}}+3 p_{\mathrm{n}}\right)\left(t-t_{s}\right)}\right)\left(1-\widehat{S}\left(n_{3}\left(t_{s}\right), n_{3}\left(t_{f}\right)\right)-n_{3}\left(t_{s}\right)\right)}{q_{\mathrm{n}}+3 p_{\mathrm{n}}-q_{\mathrm{n}}\left(1-e^{-\left(q_{\mathrm{n}}+3 p_{\mathrm{n}}\right)\left(t-t_{s}\right)}\right)\left(1-\widehat{S}\left(n_{3}\left(t_{s}\right), n_{3}\left(t_{f}\right)\right)-n_{3}\left(t_{s}\right)\right)}$,

$o_{1}(t)=\frac{M}{\mathcal{M}}\left(1-n_{3}(t)-\left(\frac{n_{3}(t)-n_{3}\left(t_{s}\right)}{p_{\mathrm{n}}+q_{\mathrm{n}} n_{3}\left(t_{s}\right)}\right)\left(2 p_{\mathrm{n}}+q_{\mathrm{n}} \widehat{S}\left(n_{3}\left(t_{s}\right), n_{3}\left(t_{f}\right)\right)\right)-\widehat{S}\left(n_{3}\left(t_{s}\right), n_{3}\left(t_{f}\right)\right)\right)$,

$o_{2}(t)=\frac{m}{\mathcal{M}}\left(1-n_{3}(t)-\left(\frac{n_{3}(t)-n_{3}\left(t_{s}\right)}{p_{\mathrm{n}}+q_{\mathrm{n}} n_{3}\left(t_{s}\right)}\right)\left(2 p_{\mathrm{n}}+q_{\mathrm{n}} \widehat{S}\left(n_{3}\left(t_{s}\right), n_{3}\left(t_{f}\right)\right)\right)-\widehat{S}\left(n_{3}\left(t_{s}\right), n_{3}\left(t_{f}\right)\right)\right)$,

$n_{1}(t)=p_{\mathrm{n}}\left(\frac{n_{3}(t)-n_{3}\left(t_{s}\right)}{p_{\mathrm{n}}+q_{\mathrm{n}} n_{3}\left(t_{s}\right)}\right), n_{2}(t)=\left(\frac{n_{3}(t)-n_{3}\left(t_{s}\right)}{p_{\mathrm{n}}+q_{\mathrm{n}} n_{3}\left(t_{s}\right)}\right)\left(p_{\mathrm{n}}+q_{\mathrm{n}} \widehat{S}\left(n_{3}\left(t_{s}\right), n_{3}\left(t_{f}\right)\right)\right)+\widehat{S}\left(n_{3}\left(t_{s}\right), n_{3}\left(t_{f}\right)\right)$,

where

$$
\widehat{S}\left(n_{3}\left(t_{s}\right), n_{3}\left(t_{f}\right)\right)=p_{\mathrm{n}}\left(\frac{n_{3}\left(t_{s}\right)-n_{3}\left(t_{f}\right)}{p_{\mathrm{n}}+q_{\mathrm{n}} n_{3}\left(t_{f}\right)}\right) .
$$

As expected, in the case where the new service model turns out to be successful, the diffusion dynamics described in the above Propositions have important implications for the values of terminal customer base percentages of competing firms, $n_{1}^{\infty}=\lim _{t \rightarrow \infty} n_{1}(t), n_{2}^{\infty}=\lim _{t \rightarrow \infty} n_{2}(t)$ and $n_{3}^{\infty}=$ $\lim _{t \rightarrow \infty} n_{3}(t)$. These terminal values are often viewed by firms themselves, as well as by investors as proxies for overall profits and as indicators of the strength of firms' competitive positions. In particular, given the multi-product nature of most established incumbents, large market share in a particular product often increases the perceived attractiveness of other components of the firm's product portfolio. In addition, a large volume of transactions in which a firm participates provides valuable market information, which can be used for more effective cross-selling of the product portfolio.

In the following Proposition, we describe the terminal customer base values for the different diffusion settings described above. We find that the presence of inter-generational churn plays a significant role in the distribution of these terminal values. 
Proposition 6. a) If there is positive intra-generational customer churn and the adoption occurs through symmetric terms $\left(Q_{n}=q_{\mathrm{n}}\right)$, the terminal customer base values of the new entrant and the incumbents are identical:

$$
m_{1}^{\infty}=m_{2}^{\infty}=m_{3}^{\infty}=\frac{\mathcal{M}}{3} .
$$

b) If there is no intra-generational customer churn $\left(p_{\mathrm{oc}}=p_{\mathrm{nc}}=0\right)$, and no market expansion resulting from the introduction of the new business model, $\mathcal{M}=M+m$, the terminal customer base value of the new entrant, $n_{3}^{\infty}$, is a root of the following equation:

$$
n_{3}^{\infty}=1-\frac{\gamma}{\delta}\left(\left(\frac{\gamma+n_{3}^{\infty}}{\gamma+n_{3}\left(t_{f}\right)}\right)^{\delta}+\left(\frac{\gamma+n_{3}^{\infty}}{\gamma+n_{3}\left(t_{s}\right)}\right)^{\delta}-2\right)
$$

while the terminal customer base values of the incumbent firms are given by

$$
n_{1}^{\infty}=\frac{\gamma}{\delta}\left(\left(\frac{\gamma+n_{3}^{\infty}}{\gamma+n_{3}\left(t_{s}\right)}\right)^{\delta}-1\right), n_{2}^{\infty}=\frac{\gamma}{\delta}\left(\left(\frac{\gamma+n_{3}^{\infty}}{\gamma+n_{3}\left(t_{f}\right)}\right)^{\delta}-1\right)
$$

where $n_{3}\left(t_{f}\right)$ and $n_{3}\left(t_{s}\right)$ are the customer base values for the new entrant firm at times $t_{f}$ and $t_{s}$ at which firms 1 and 2, respectively, adopt the new service model, and $\gamma=\frac{p_{\mathrm{n}}}{q_{\mathrm{n}}}, \delta=\frac{Q_{\mathrm{n}}}{q_{\mathrm{n}}}$. In particular, in a homogeneous imitation setting $(\delta=1)$, the terminal customer base values are

$$
\begin{aligned}
n_{3}^{\infty} & =\frac{\left.(3 \gamma+1)\left(\gamma+n_{3}\left(t_{f}\right)\right)\right)\left(\gamma+n_{3}\left(t_{s}\right)\right)}{\left(\gamma+n_{3}\left(t_{f}\right)\right)\left(\gamma+n_{3}\left(t_{s}\right)\right)+\gamma\left(2 \gamma+n_{3}\left(t_{s}\right)+n_{3}\left(t_{f}\right)\right)}-\gamma, \\
n_{1}^{\infty} & =\frac{\left.\gamma(3 \gamma+1)\left(\gamma+n_{3}\left(t_{f}\right)\right)\right)}{\left(\gamma+n_{3}\left(t_{f}\right)\right)\left(\gamma+n_{3}\left(t_{s}\right)\right)+\gamma\left(2 \gamma+n_{3}\left(t_{s}\right)+n_{3}\left(t_{f}\right)\right)}-\gamma, \\
n_{2}^{\infty} & =\frac{\left.\gamma(3 \gamma+1)\left(\gamma+n_{3}\left(t_{s}\right)\right)\right)}{\left(\gamma+n_{3}\left(t_{f}\right)\right)\left(\gamma+n_{3}\left(t_{s}\right)\right)+\gamma\left(2 \gamma+n_{3}\left(t_{s}\right)+n_{3}\left(t_{f}\right)\right)}-\gamma .
\end{aligned}
$$

Figure 2 shows the terminal customer base values in the setting where both incumbents enter at the same time, determined by the customer base of the new entrant. The two sets of curves on this figure correspond to different relative "speeds" of the incumbent diffusion, $Q_{n}=\delta q_{n}$ with $\delta=1$ and $\delta=5$. Note that in the case of symmetric imitation $(\delta=1)$, the simultaneous entry of the new entrant and of the incumbents splits the new service model market into three equal parts; on the other hand, if incumbents wait until the new entrant captures $25 \%$ of the market before adopting, their terminal customer base values of the new market will rise only up to around $10 \%$ each, leaving 


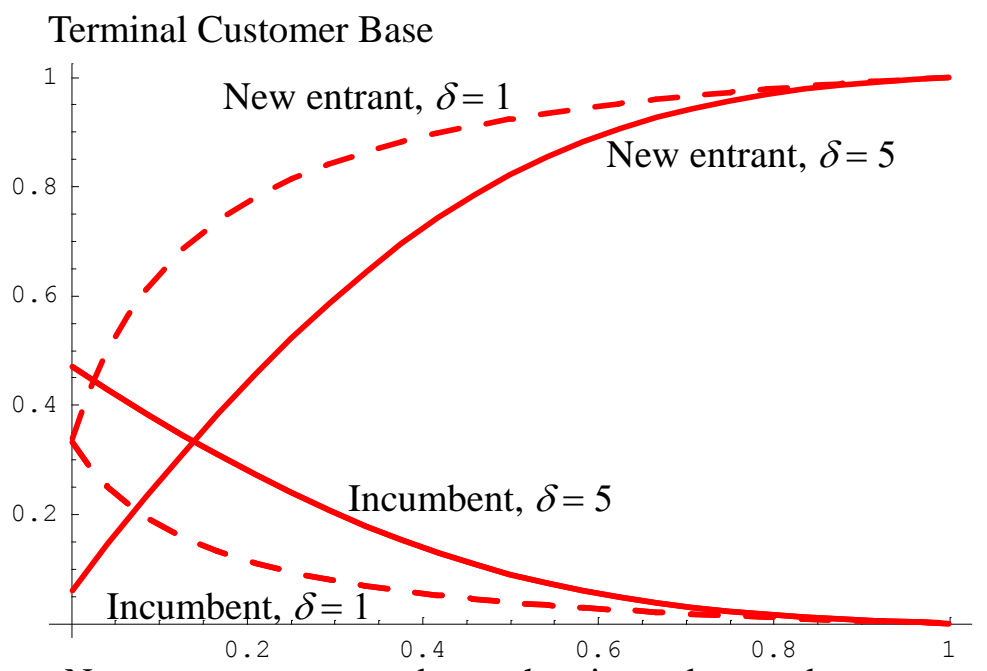

New entrant customer base when incumbents adopt

Figure 2 Terminal customer base values when both incumbents enter at the same time $(\gamma=0.05, \delta=1,5)$

$80 \%$ for the new entrant. As expected, the setting with fast incumbent diffusion $(\delta=5)$ is more forgiving to incumbent delays: incumbents can allow the new entrant to capture $15 \%$ of the total customer base, and still catch up with it in terms of terminal customer base value. In a similar manner, the new entrant will have to capture around half of the potential market before adoption by incumbents in order to keep their terminal customer base values at a $10 \%$ level.

\subsection{Phase IV: "Two Incumbents"}

The analysis above pertains to the case where the new service model turns out to be successful. If, however, the new model fails at $t=T_{F}$, then, starting at that point in time, the only firms present in the market are incumbents, both of them employing the old service model. Below we consider the diffusion dynamics in such a case for $t \geq T_{F}$. The diffusion dynamics for this time period is described by the following set of differential equations:

$$
\begin{aligned}
\frac{d m_{1}^{\mathrm{o}}}{d t} & =\left(p_{\mathrm{o}}+\frac{q_{\mathrm{o}}}{\mathcal{M}} m_{1}^{\mathrm{o}}\right)\left(\mathcal{M}-m_{1}^{\mathrm{o}}-m_{2}^{\mathrm{o}}\right)+p_{\mathrm{oc}} m_{2}^{\mathrm{o}}-p_{\mathrm{oc}} m_{1}^{\mathrm{o}}, \\
\frac{d m_{2}^{\mathrm{o}}}{d t} & =\left(p_{\mathrm{o}}+\frac{q_{\mathrm{o}}}{\mathcal{M}} m_{2}^{\mathrm{o}}\right)\left(\mathcal{M}-m_{1}^{\mathrm{o}}-m_{2}^{\mathrm{o}}\right)+p_{\mathrm{oc}} m_{1}^{\mathrm{o}}-p_{\mathrm{oc}} m_{2}^{\mathrm{o}},
\end{aligned}
$$

augmented by the initial conditions following from the solution of phase III equations, $m_{1}^{\circ}\left(T_{F}\right)$ and $m_{2}^{\circ}\left(T_{F}\right)$. The dynamics expressed by (54)-(55) with these initial conditions admit a closed-form characterization. 
Proposition 7. Consider the setting where the new model fails at $t=T_{F}$, and define, for $t \geq T_{F}$

$$
\begin{aligned}
\bar{I}_{1}(t)= & \frac{1}{2 p_{\mathrm{oc}}}\left(e^{2 p_{\mathrm{oc}}\left(t-T_{F}\right)}{ }_{2} F_{1}\left(\frac{2 p_{\mathrm{oc}}}{2 p_{\mathrm{o}}+q_{\mathrm{o}}},-\frac{1}{2 p_{\mathrm{o}}+q_{\mathrm{o}}} ; 1+\frac{2 p_{\mathrm{oc}}}{2 p_{\mathrm{o}}+q_{\mathrm{o}}} ;\left(-\frac{\frac{q_{\mathrm{o}}}{\mathcal{M}}\left(\mathcal{M}-m^{\mathrm{o}}\left(T_{F}\right)\right)}{2 p_{\mathrm{o}}+\frac{q_{\mathrm{o}}}{\mathcal{M}} m^{\mathrm{o}}\left(T_{F}\right)}\right)\left(e^{-\left(2 p_{\mathrm{o}}+q_{\mathrm{o}}\right)\left(t-T_{F}\right)}\right)\right)\right. \\
& \left.-{ }_{2} F_{1}\left(\frac{2 p_{\mathrm{oc}}}{2 p_{\mathrm{o}}+q_{\mathrm{o}}},-\frac{1}{2 p_{\mathrm{o}}+q_{\mathrm{o}}} ; 1+\frac{2 p_{\mathrm{oc}}}{2 p_{\mathrm{o}}+q_{\mathrm{o}}} ;\left(-\frac{\frac{q_{\mathrm{o}}}{\mathcal{M}}\left(\mathcal{M}-m^{\mathrm{o}}\left(T_{F}\right)\right)}{2 p_{\mathrm{o}}+\frac{q_{\mathrm{o}}}{\mathcal{M}} m^{\mathrm{o}}\left(T_{F}\right)}\right)\right)\right)
\end{aligned}
$$

and

$$
\begin{aligned}
\bar{I}_{2}(t)= & \frac{1}{2 p_{\mathrm{oc}}}\left(e^{2 p_{\mathrm{oc}}\left(t-T_{F}\right)}{ }_{2} F_{1}\left(\frac{2 p_{\mathrm{oc}}}{2 p_{\mathrm{o}}+q_{\mathrm{o}}}, 1-\frac{1}{2 p_{\mathrm{o}}+q_{\mathrm{o}}} ; 1+\frac{2 p_{\mathrm{oc}}}{2 p_{\mathrm{o}}+q_{\mathrm{o}}} ;\left(-\frac{\frac{q_{\mathrm{o}}}{\mathcal{M}}\left(\mathcal{M}-m^{\mathrm{o}}\left(T_{F}\right)\right)}{2 p_{\mathrm{o}}+\frac{q_{\mathrm{o}}}{\mathcal{M}} m^{\mathrm{o}}\left(T_{F}\right)}\right)\left(e^{-\left(2 p_{\mathrm{o}}+q_{\mathrm{o}}\right)\left(t-T_{F}\right)}\right)\right)\right. \\
& \left.-{ }_{2} F_{1}\left(\frac{2 p_{\mathrm{oc}}}{2 p_{\mathrm{o}}+q_{\mathrm{o}}}, 1-\frac{1}{2 p_{\mathrm{o}}+q_{\mathrm{o}}} ; 1+\frac{2 p_{\mathrm{oc}}}{2 p_{\mathrm{o}}+q_{\mathrm{o}}} ;\left(-\frac{\frac{q_{\mathrm{o}}}{\mathcal{M}}\left(\mathcal{M}-m^{\mathrm{o}}\left(T_{F}\right)\right)}{2 p_{\mathrm{o}}+\frac{q_{\mathrm{o}}}{\mathcal{M}} m^{\mathrm{o}}\left(T_{F}\right)}\right)\right)\right),
\end{aligned}
$$

where $m_{i}^{\circ}\left(T_{F}\right), i=1,2$ is the customer base of incumbent $i$ at time $T_{F}$, and $m^{\circ}\left(T_{F}\right)=m_{1}^{\circ}\left(T_{F}\right)+$ $m_{2}^{\circ}\left(T_{F}\right)$. Then, the customer bases of the incumbents for $t \geq T_{F}$ are given by

$$
\begin{aligned}
& m_{i}^{\circ}(t)=e^{-2 p_{\mathrm{oc}}\left(t-T_{F}\right)}\left(\frac{1+\left(\frac{\frac{q_{\mathrm{O}}}{\mathcal{M}}\left(\mathcal{M}-m^{\circ}\left(T_{F}\right)\right)}{2 p_{\mathrm{o}}+\frac{q_{\mathrm{o}}}{\mathcal{M}} m^{\circ}\left(T_{F}\right)}\right)}{1+\left(\frac{\frac{q_{\mathrm{o}}}{\mathcal{M}}\left(\mathcal{M}-m^{\circ}\left(T_{F}\right)\right)}{2 p_{\mathrm{o}}+\frac{q_{\mathrm{o}}}{\mathcal{M}} m^{\circ}\left(T_{F}\right)}\right) e^{-\left(2 p_{\mathrm{o}}+q_{\mathrm{o}}\right)\left(t-T_{F}\right)}}\right)^{\frac{1}{2 p_{\mathrm{o}}+q_{\mathrm{o}}}} \\
& \times\left(m_{i}^{\circ}\left(T_{F}\right)\right.
\end{aligned}
$$

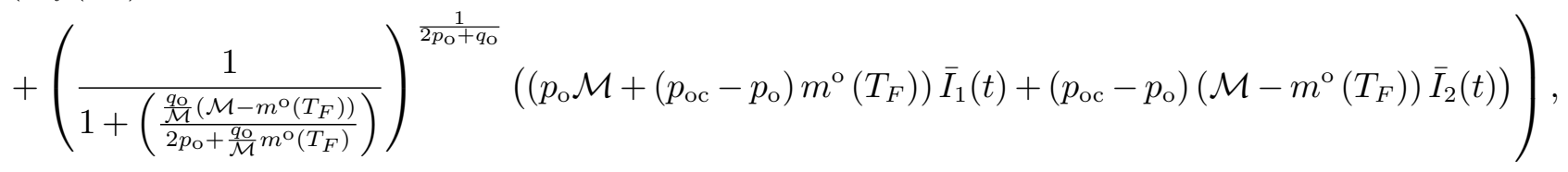

$$
\begin{aligned}
& i=1,2 \text {. }
\end{aligned}
$$

The results of the Proposition 7 indicate, in particular, that $\lim _{t \rightarrow+\infty} m_{i}^{\circ}(t)=\frac{\mathcal{M}}{2}, i=1,2$ : upon the failure of the new model, the diffusion dynamics reflects the competition between the incumbents that end up, in the long run, splitting the market equally. This result is a direct consequence of our assumption on the symmetry of the process of adoption of the old service model.

\subsection{Terminal Customer Base Values in the Settings with Asymmetric Churn}

The terminal customer base values serve as important indicators of relative strength of competing firms in settings where the new model succeeds as well as in settings where the new model fails. In the former case, the terminal customer base values reflect the relative strength of the incumbents under the old model, while in the latter - the relative strength of the newcomer and the incumbents under the new model. Below we derive the expressions for the terminal customer base values in 
both cases in the general setting with asymmetric churn among the incumbents employing the old model as well as the asymmetric churn between the incumbents and the newcomer when they all employ the new model. In particular, we assume that the diffusion dynamics in the general setting with asymmetric churn with three firms present is described by

$$
\begin{aligned}
\frac{d m_{1}^{\mathrm{o}}}{d t}= & \left(p_{\mathrm{o}}+\frac{q_{\mathrm{o}}}{\mathcal{M}} m_{1}^{\mathrm{o}}\right)\left(\mathcal{M}-m_{1}^{\mathrm{o}}-m_{2}^{\mathrm{o}}-m_{1}^{\mathrm{n}}-m_{2}^{\mathrm{n}}-m_{3}^{\mathrm{n}}\right)+p_{\mathrm{oc}}^{12} m_{2}^{\mathrm{o}} \\
& -\left(p_{\mathrm{oc}}^{21}+3 p_{\mathrm{n}}+\frac{Q_{\mathrm{n}}}{\mathcal{M}}\left(m_{1}^{\mathrm{n}}+m_{2}^{\mathrm{n}}\right)+\frac{q_{\mathrm{n}}}{\mathcal{M}} m_{3}^{\mathrm{n}}\right) m_{1}^{\mathrm{o}}, \\
\frac{d m_{2}^{\mathrm{o}}}{d t}= & \left(p_{\mathrm{o}}+\frac{q_{\mathrm{o}}}{\mathcal{M}} m_{2}^{\mathrm{o}}\right)\left(\mathcal{M}-m_{1}^{\mathrm{o}}-m_{2}^{\mathrm{o}}-m_{1}^{\mathrm{n}}-m_{2}^{\mathrm{n}}-m_{3}^{\mathrm{n}}\right)+p_{\mathrm{oc}}^{21} m_{1}^{\mathrm{o}} \\
& -\left(p_{\mathrm{oc}}^{12}+3 p_{\mathrm{n}}+\frac{Q_{\mathrm{n}}}{\mathcal{M}}\left(m_{1}^{\mathrm{n}}+m_{2}^{\mathrm{n}}\right)+\frac{q_{\mathrm{n}}}{\mathcal{M}} m_{3}^{\mathrm{n}}\right) m_{2}^{\mathrm{o}}, \\
\frac{d m_{1}^{\mathrm{n}}}{d t}= & \left(p_{\mathrm{n}}+\frac{Q_{\mathrm{n}}}{\mathcal{M}} m_{1}^{\mathrm{n}}\right)\left(\mathcal{M}-m_{1}^{\mathrm{n}}-m_{2}^{\mathrm{n}}-m_{3}^{\mathrm{n}}\right)+p_{\mathrm{nc}}^{12} m_{2}^{\mathrm{n}}+p_{\mathrm{nc}}^{13} m_{3}^{\mathrm{n}}-\left(p_{\mathrm{nc}}^{21}+p_{\mathrm{nc}}^{31}\right) m_{1}^{\mathrm{n}}, \\
\frac{d m_{2}^{\mathrm{n}}}{d t}= & \left(p_{\mathrm{n}}+\frac{Q_{\mathrm{n}}}{\mathcal{M}} m_{2}^{\mathrm{n}}\right)\left(\mathcal{M}-m_{1}^{\mathrm{n}}-m_{2}^{\mathrm{n}}-m_{3}^{\mathrm{n}}\right)+p_{\mathrm{nc}}^{21} m_{1}^{\mathrm{n}}+p_{\mathrm{nc}}^{23} m_{3}^{\mathrm{n}}-\left(p_{\mathrm{nc}}^{12}+p_{\mathrm{nc}}^{32}\right) m_{2}^{\mathrm{n}}, \\
\frac{d m_{3}^{\mathrm{n}}}{d t}= & \left(p_{\mathrm{n}}+\frac{q_{\mathrm{n}}}{\mathcal{M}} m_{3}^{\mathrm{n}}\right)\left(\mathcal{M}-m_{1}^{\mathrm{n}}-m_{2}^{\mathrm{n}}-m_{3}^{\mathrm{n}}\right)+p_{\mathrm{nc}}^{31} m_{1}^{\mathrm{n}}+p_{\mathrm{nc}}^{32} m_{2}^{\mathrm{n}}-\left(p_{\mathrm{nc}}^{13}+p_{\mathrm{nc}}^{23}\right) m_{3}^{\mathrm{n}},
\end{aligned}
$$

where $p_{\mathrm{nc}}^{i j}>0$ is the parameter that describes the churn from the customer group that uses the new model offered by the firm $j$ to the customer group that uses the new model offered by firm $i \neq j$, and $p_{\mathrm{oc}}^{i j}>0$ is the parameter that describes the churn from the customer group that uses the old model offered by the firm $j$ to the customer group that uses the old model offered by firm $i \neq j$.

The terminal customer base values of competing firms are expressed by the following result.

Proposition 8. a) In a setting where the new model succeeds, the terminal customer base values corresponding to the old service model, $o_{i}^{\infty}=\lim _{t \rightarrow \infty} o_{i}(t), i=1,2$ are zero, while the terminal customer base values corresponding to the new technology, $n_{i}^{\infty}=\lim _{t \rightarrow \infty} n_{i}(t), i=1,2,3$, are given by

$$
\begin{aligned}
& n_{1}^{\infty}=\frac{p_{\mathrm{nc}}^{12} p_{\mathrm{nc}}^{23}+p_{\mathrm{nc}}^{13} p_{\mathrm{nc}}^{32}+p_{\mathrm{nc}}^{12} p_{\mathrm{nc}}^{13}}{p_{\mathrm{nc}}^{12} p_{\mathrm{nc}}^{23}+p_{\mathrm{nc}}^{21} p_{\mathrm{nc}}^{13}+p_{\mathrm{nc}}^{31} p_{\mathrm{nc}}^{12}+p_{\mathrm{nc}}^{23} p_{\mathrm{nc}}^{31}+p_{\mathrm{nc}}^{13} p_{\mathrm{nc}}^{32}+p_{\mathrm{nc}}^{32} p_{\mathrm{nc}}^{21}+p_{\mathrm{nc}}^{12} p_{\mathrm{nc}}^{13}+p_{\mathrm{nc}}^{21} p_{\mathrm{nc}}^{23}+p_{\mathrm{nc}}^{31} p_{\mathrm{nc}}^{32}} \\
& n_{2}^{\infty}=\frac{p_{\mathrm{nc}}^{21} p_{\mathrm{nc}}^{13}+p_{\mathrm{nc}}^{23} p_{\mathrm{nc}}^{31}+p_{\mathrm{nn}}^{21} p_{\mathrm{nc}}^{23}}{p_{\mathrm{nc}}^{12} p_{\mathrm{nc}}^{23}+p_{\mathrm{nc}}^{21} p_{\mathrm{nc}}^{13}+p_{\mathrm{nc}}^{31} p_{\mathrm{nc}}^{12}+p_{\mathrm{nc}}^{23} p_{\mathrm{nc}}^{31}+p_{\mathrm{nc}}^{13} p_{\mathrm{nc}}^{32}+p_{\mathrm{nc}}^{32} p_{\mathrm{nc}}^{21}+p_{\mathrm{nc}}^{12} p_{\mathrm{nc}}^{13}+p_{\mathrm{nc}}^{21} p_{\mathrm{nc}}^{23}+p_{\mathrm{nc}}^{31} p_{\mathrm{nc}}^{32}} \\
& n_{3}^{\infty}=\frac{p_{\mathrm{nc}}^{32} p_{\mathrm{nc}}^{21}+p_{\mathrm{nc}}^{31} p_{\mathrm{nc}}^{12}+p_{\mathrm{nc}}^{31} p_{\mathrm{nc}}^{32}}{p_{\mathrm{nc}}^{12} p_{\mathrm{nc}}^{23}+p_{\mathrm{nc}}^{21} p_{\mathrm{nc}}^{13}+p_{\mathrm{nc}}^{31} p_{\mathrm{nc}}^{12}+p_{\mathrm{nc}}^{23} p_{\mathrm{nc}}^{31}+p_{\mathrm{nc}}^{13} p_{\mathrm{nc}}^{32}+p_{\mathrm{nc}}^{32} p_{\mathrm{nc}}^{21}+p_{\mathrm{nc}}^{12} p_{\mathrm{nc}}^{13}+p_{\mathrm{nc}}^{21} p_{\mathrm{nc}}^{23}+p_{\mathrm{nc}}^{31} p_{\mathrm{nc}}^{32}}
\end{aligned}
$$


b) In the setting where the new model fails, the terminal customer base values corresponding to the old service model are given by

$$
\begin{aligned}
o_{1}^{\infty} & =\frac{p_{\mathrm{oc}}^{21}}{p_{\mathrm{oc}}^{12}+p_{\mathrm{oc}}^{21}}, \\
o_{2}^{\infty} & =\frac{p_{\mathrm{oc}}^{12}}{p_{\mathrm{oc}}^{12}+p_{\mathrm{oc}}^{21}} .
\end{aligned}
$$

Part b) of Proposition 8 outlines the impact of asymmetry in the inter-incumbent churn processes on the way the market is split when all potential customers have made their adoption decisions in the setting where the old model manages to stave off the innovation threat. If the new model fails, the ratio of incumbents' relative customer bases is equal to the ratio of their churn parameters. Part a) of Proposition 8 describes an opposing setting where the new model survives and completely replaces the old one. In this setting, the segmentation of the market dominated by the new model follows a much more complex pattern. The terminal customer base expressions (64)-(66) reflect the most general, and entirely asymmetric, churn dynamics between the incumbents and the new entrant. Note that, in the trivial special case where all churn coefficients are equal, the three firms, as expected, split the market equally. Figure 3 provides an illustration of the terminal market coverage in the case where the churn processes between the incumbents and the new entrant are symmetric (i.e, $p_{\mathrm{nc}}^{13}=p_{\mathrm{nc}}^{31}=p_{\mathrm{nc}}^{23}=p_{\mathrm{nc}}^{32}=\hat{p}$ ), but the churn process between the incumbents is not (so that, in general, $p_{\mathrm{nc}}^{12} \neq p_{\mathrm{nc}}^{21} \neq \hat{p}$ ). Figure 3 a looks at the subcase where the churn between the incumbents and the new entrant is very slow $\left(\hat{p}=0.01<<p_{\mathrm{nc}}^{12}=1\right)$, while the Figure $3 \mathrm{~b}$ describes the subcase where the churn between the incumbents and the new entrant is very fast $(\hat{p}=100>>$ $\left.p_{\mathrm{nc}}^{12}=1\right)$. There are two important observations that can be made in such semi-asymmetric setting. First, the symmetry in the incumbent-new entrant churn ensures that the customer base of the new entrant is equal to $\frac{1}{3}$, and is not affected by the intra-incumbent churn processes. Second, the way the incumbents split the remaining $\frac{2}{3}$ of the market can be strongly affected by the presence of the new entrant. Note that, as (64)-(65) imply, for $p_{\mathrm{nc}}^{13}=p_{\mathrm{nc}}^{31}=p_{\mathrm{nc}}^{23}=p_{\mathrm{nc}}^{32}=\hat{p}$ the ratio of the terminal customer base values for the two incumbents is

$$
\frac{n_{1}^{\infty}}{n_{2}^{\infty}}=\frac{p_{\mathrm{nc}}^{12}+2 \hat{p}}{p_{\mathrm{nc}}^{21}+2 \hat{p}}
$$



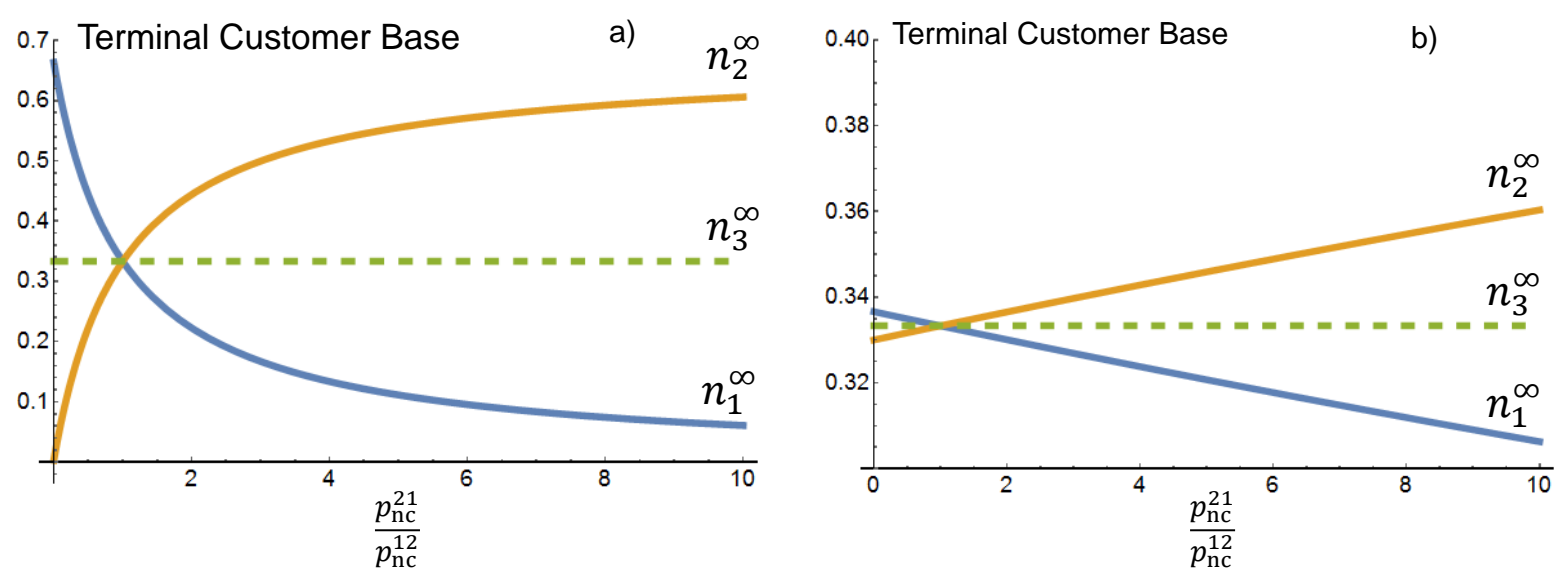

Figure 3 : The terminal customer base values as functions of the incumbent churn assymetry factor $\frac{p_{\mathrm{nc}}^{21}}{p_{\mathrm{nc}}^{12}}$ for different values of new entrant-incumbent churn parameter $\hat{p}=p_{\mathrm{nc}}^{13}=p_{\mathrm{nc}}^{31}=p_{\mathrm{nc}}^{23}=p_{\mathrm{nc}}^{32}$ : a) $\left.\hat{p}=0.01, p_{\mathrm{nc}}^{12}=1, \mathbf{b}\right)$ $\hat{p}=100, p_{\mathrm{nc}}^{12}=1$.

Comparing this expression with the ratio justified by the intra-incumbent churn processes alone, $\frac{p_{\text {nc }}^{12}}{p_{\mathrm{nc}}^{21}}$, we observe that $(69)$ is greater than $\frac{p_{\mathrm{nc}}^{12}}{p_{\mathrm{nc}}^{21}}$ if and only if $\frac{p_{\mathrm{nc}}^{12}}{p_{\mathrm{nc}}^{21}}<1$. Note that if $p_{\mathrm{nc}}^{12}<p_{\mathrm{nc}}^{21}$, the incumbent 1 is less "attractive" to consumers than the incumbent 2 and, therefore, has smaller terminal customer base values in the absence of a newcomer. Thus, the presence of the new entrant allows for the incumbent 1 to have higher terminal customer base. As (69) indicates, such moderating effect of the new entrant becomes especially pronounced if the churn that involves the new entrant dominates other churn processes.

\section{An Empirical Example: FX market}

In this section, we illustrate the application of our model by examining the empirical context mentioned briefly in the Introduction section, namely, the foreign exchange (FX) market. Obtaining detailed firm-level data to illustrate our model is not trivial. Most previous studies of new product diffusion have been conducted at the category level (Libai et al. 2009a, p. 24). We have collected our data from archival material (press releases and industry reports), and supplemented our data with detailed interviews with senior managers in the industry.

The foreign exchange market displays four characteristic that make it appropriate context in which to illustrate the application of our model. First, this market had a traditional service model 
with the potential to be transformed into a new one. Second, there was a new entrant that launched the new service model. Third, incumbent firms with different sizes of customer bases existed. Fourth, the markets displayed different adoption times for the innovation by the major and minor firms respectively.

The major customers in the foreign exchange market are corporations such as investment management firms, pension funds and insurance firms. Customers typically use the foreign exchange market for international trade, cross-border investments and hedging against currency risks. Traditionally, the trading on the foreign exchange market was done in an over-the-counter mode whereby the customer had to make several phone calls to dealers to obtain quotes. Dealers, in turn, offered both buy and sell quotes to customers. Once a price has been agreed upon, the transaction was executed with one of the dealer banks. In the traditional process, the dealers' business model was based on generating revenues from the spread between buy and sell prices. Dealers are able to charge a spread because of their ability to match buyers and sellers as well as to take on inventory risks. Often dealers might not be able to match buyers and sellers at the same time and hence, would need to hold the currencies in inventory until a corresponding customer is found.

The advent of the Internet enabled more efficient means for price information dissemination and execution of trades via electronic trading technology. In this new service model, dealers post their bid and offer prices, or customers can request quotes from multiple dealers and transact accordingly on the electronic platform. In April 2000, Currenex, a new entrant, launched a new model of foreign exchange trading via the Internet. Currenex started attracting customers away from the incumbent dealer banks onto its platform. In response, the dealer banks formed two consortia to adopt the new foreign exchange trading model. The first consortium consisted of three of the world's largest dealers in foreign exchange, namely Citibank, JP Morgan Chase and Deutsche Bank, with the combined share of $28.9 \%$ of total daily trading volume; the second consortium was formed by seven smaller/minor banks with the combined daily trading volume adding up to $25.5 \%$ of the total (Euromoney 2000). Thus, from the point of view of the adoption of the new business model the 
dealer banks essentially acted like a duopoly. The consortium led by the minor banks adopted the new business model by launching a platform called FXall in May 2001 (13 months after the new entrant). This was then followed by the consortium led by the major banks launching a similar platform, Atriax in June 2001 (14 months after the new entrant). Atriax, however, stopped its operation in April 2002.

We have estimated the overall market potential $\mathcal{M}$ as well as the innovation and imitation parameters using the average daily trading volume data for competing bank consortia shown in Appendix B. Since the numbers of customer accounts are not publicly available, in our estimation analysis we used the daily transaction volume as a proxy for the sizes of customer bases in foreign exchange e-trading. In addition, since all of the available data estimates relate to the trading volumes for the new service model, we have used the estimate for the total daily trading volume at around the time of the introduction of the new model (\$1 trillion, Reuters News 2001) and the pre-innovation customer base estimates for the major and the minor incumbents to set their respective values at $M=\$ 289$ billion, and $m=\$ 255$ billion, and focused on establishing the values for $\mathcal{M}, p_{\mathrm{o}}, q_{\mathrm{o}}, p_{\mathrm{oc}}, p_{\mathrm{n}}, q_{\mathrm{n}}, Q_{\mathrm{n}}$ and $p_{\mathrm{nc}}$ (8 parameters) that provide the best fit for the available data.

We established the best-fit parameters by minimizing the sum of the equally-weighted squared deviations between the analytical and the actual customer bases values. For the fitting procedure, we have used the discretized version of our model, with the discrete time unit equal to 1 month to reflect the empirical data. To account for the finite time that the major incumbent invested in the Atriax platform, we have set the customer bases values corresponding to the new model offered by the major incumbent to 0 after April 2002, thus estimating the model where the minor incumbent and the new entrant share the new model market. The minimization was done using Excel Solver with "GRG Nonlinear" option with multiple random restarts. After obtaining the best-fit values, we have run the "Evolutionary Solver" on the resulting values to ensure that they cannot be improved upon. The resulting estimated values are reported in Table 2 .

Figure 4 illustrates the fit between the analytical model with parameters from the Table 2 and the empirical data for Currenex, the new entrant (Figure 4a) and FXAll, the minor incumbent (Figure 4b). 


\begin{tabular}{|c|c|}
\hline Parameter & Estimated Value, $\$$ bn \\
\hline $\mathcal{M}$ & 544.00 \\
\hline \hline Parameter & Estimated Value, $\times 10^{-5} \frac{1}{\text { month }}$ \\
\hline$p_{\mathrm{o}}$ & 8.74 \\
\hline$q_{\mathrm{o}}$ & 0.23 \\
\hline$p_{\text {oc }}$ & 96.23 \\
\hline \hline Parameter & Estimated Value, $\times 10^{-5} \frac{1}{\text { month }}$ \\
\hline$p_{\mathrm{n}}$ & 1.10 \\
\hline$q_{\mathrm{n}}$ & 7233.87 \\
\hline$Q_{\mathrm{n}}$ & 1645.64 \\
\hline$p_{\mathrm{nc}}$ & 0.00 \\
\hline
\end{tabular}

Table 2 The best-fit parameter values.

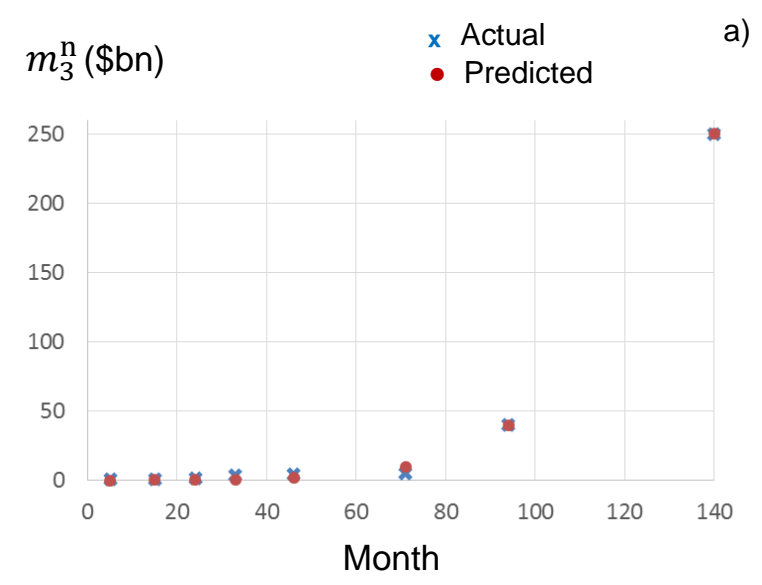

a)

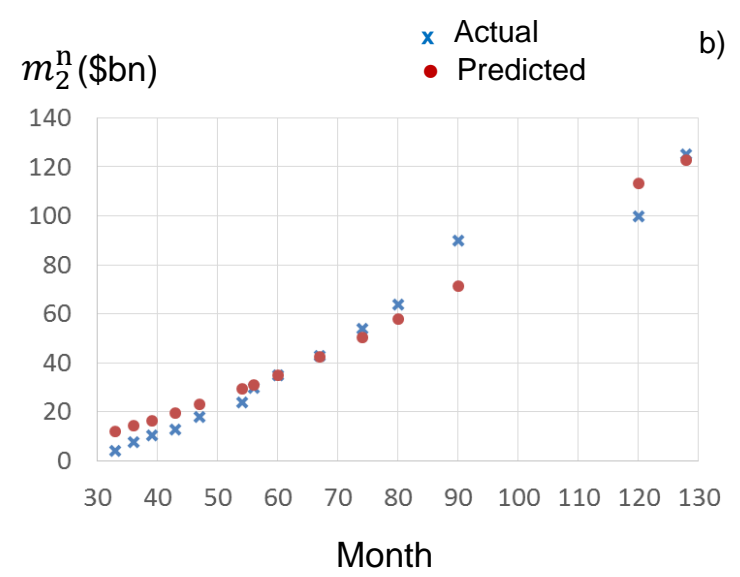

Figure 4 : The actual vs. predicted average daily trading volumes a) for the new entrant (Currenex), and b) for the minor incumbent (FXAII).

Table 2 prompts several observations regarding the diffusion dynamics in the environment described by the FX trading data. First, the introduction of a new service model has resulted, in this environment, in a redistribution of the existing market $(\mathcal{M}=M+m)$. Second, the customer base dynamics under the old service model and under the new service model appear to have very 
different features: the old model is driven mostly by innovation in the presence of a strong customer churn, while the new model expands almost exclusively through imitation, with no customer churn. Finally, the new entrant have a clear first-mover advantage in terms of the speed of the "imitation" process as compared to the incumbents. In Section 5 we will use the best-fit estimates from Table 2 to conduct a numerical study of the impact of these features of the customer base dynamics on the emerging Nash equilibria in adoption of the new service model by the incumbent firms.

\section{Optimal Response Decisions and Nash Equilibria Analysis: Successful Innovation in the Absence of Market Expansion or Intra-Generational Churn}

In this section we analyze the optimal response and the equilibrium entry-time decisions of the incumbents. While under the general model the analysis of the equilibrium market-entry decisions appears intractable, we managed to provide characterization of such decisions for the special setting where the introduction of the new service model is guaranteed to be successful (i.e. $P_{F}=0$ ), does not expand the overall market $(\mathcal{M}=M+m)$, and there is no intra-generational churn $\left(p_{\mathrm{oc}}=p_{\mathrm{nc}}=0\right)$, as described in Corollary 1.2 and Propositions 3 and 5.

The analysis of the competitive diffusion dynamics developed in the previous section connects the new service model adoption decisions by two incumbent firms with the resulting customer base trajectories. Below we adopt a perspective of one of the incumbent firms and use these trajectories to build a framework for making the optimal adoption decisions. Without loss of generality, we select firm 1, and assume that the time $t_{2}$ at which the other incumbent, firm 2, commits to adopting the new service model is known. We also assume that firm 1's objective is to maximize its discounted profits $\Pi_{1}$ over the infinite planning horizon:

$$
\Pi_{1}\left(t_{1}, t_{2}\right)=\int_{0}^{+\infty} e^{-\beta t}\left(\pi_{\mathrm{o}} o_{1}(t)+\pi_{\mathrm{n}} n_{1}(t)\right) d t,
$$

where $\pi_{\mathrm{o}}\left(\pi_{\mathrm{n}}\right)$ is a per-unit-of-time profit contribution from each firm 1 customer using old (new) service model, and $\beta$ is the time discounting factor. Note that, in practice, the profit contributions themselves may be affected by the respective customer base values. To maintain the tractability of 
the analysis of the adoption times, we use the simplified approach that assigns to the profit margins constant values that reflect the average profit margins earned over a large range of customer base values.

For simplicity, we consider the adoption times to be between 0 ("adopt immediately") and some maximum time $t_{\max }$ ("delay adoption as much as possible"). In other words, the profit maximization problem for firm 1 can be expressed as

$$
\max _{0 \leq t_{1} \leq t_{\max }} \Pi_{1}\left(t_{1}, t_{2}\right)
$$

so that the optimal adoption response time is defined as

$$
t_{1}^{\mathrm{opt}}=\arg \max _{0 \leq t_{1} \leq t_{\max }} \Pi_{1}\left(t_{1}, t_{2}\right)
$$

The choice of $t_{\max }$, while somewhat arbitrary, can be tied to the time characteristic of the customer base dynamics of the new firm $n_{3}(t)$ in the first phase of the competitive diffusion: the rate of change of $n_{3}(t)$, as shown in Bass (1969), peaks at $T=\frac{1}{p_{\mathrm{n}}+q_{\mathrm{n}}} \ln \left(\frac{q_{\mathrm{n}}}{p_{\mathrm{n}}}\right)$ and becomes negligible after $2 T$. Thus, setting, for example, $t_{\max }=\frac{3}{p_{\mathrm{n}}+q_{\mathrm{n}}} \ln \left(\frac{q_{\mathrm{n}}}{p_{\mathrm{n}}}\right)$ appears to be a reasonable choice. The optimization problem (71) is well-behaved under a mild assumption on the time parameters of the underlying dynamics:

Proposition 9. For $\beta \leq 3 p_{\mathrm{n}}$ the profit $\Pi_{1}\left(t_{1}, t_{2}\right)$ is a quasiconcave function of $t_{1}$.

The assumption of Proposition 9, ensuring that the profit function of firm 1 has unique local maximizing adoption time, is both mild and easy-to-interpret: in order for the profit function to be well-behaved, we require that the process of time discounting of profits proceeds at a relatively slow rate and does not interfere with the dynamics of adoption of the new service model. Such an assumption seems quite reasonable in cases of computer/Internet-related technology diffusions, since it is likely that such "digital diffusions" exhibit much faster innovation dynamics than traditional ones (Rangaswamy and Gupta 1999). For example, Lilien, Rangaswamy and Van den Bulte (2000) report the innovation parameter $p_{\mathrm{n}}=0.121$ per year for PC adoptions in the US - as compared to the average of $p_{\mathrm{n}}=0.037$ across multiple categories of agricultural, medical equipment, 
production technology and consumer electronics products. For a diffusion similar to the PC adoption, the maximum value of time discounting factor $\beta$ satisfying the assumption of Proposition 9 is $3 p_{\mathrm{n}}=36.3 \%$ per year, which is high enough to accommodate any realistic discount rate. While in real business settings the value of $\beta$ is likely to exceed this level, our extensive numerical tests show that the quasiconcavity of the profit function is preserved even for much higher values of time discounting factor.

For a given value of $t_{2}$, the optimal response time $t_{1}^{\text {opt }}$ depends on the underlying parameters of the diffusion problem, in particular, on the values of firm 1's initial customer base $\theta$, as well as the profit contributions $\pi_{\mathrm{o}}$ and $\pi_{\mathrm{n}}$. The monotonicity properties of the response time $t_{1}^{\mathrm{opt}}$ are formalized by the following Proposition:

Proposition 10. The optimal response time $t_{1}^{\text {opt }}$ is a non-decreasing function of the profitadjusted customer base $\frac{\pi_{o} \theta}{\pi_{n}}$.

Proposition 10 indicates that high initial customer base predisposes a firm to delay its innovation response.

The equilibrium analysis of the adoption decisions in a competitive environment can be recast in terms of finding a pair of Nash equilibrium adoption times $t_{1}^{*}$ and $t_{2}^{*}$ such that $t_{1}^{*}=t_{1}^{\text {opt }}\left(t_{2}^{*}\right)$ and $t_{2}^{*}=t_{2}^{\text {opt }}\left(t_{1}^{*}\right)$. In a more general setting the equilibrium analysis may also include the endogenous determination of the diffusion parameters. While the existence of pure equilibrium in terms of adoption times is not guaranteed, mixed equilibrium strategies for the model we analyze do exist for any combination of problem parameters, as implied by the continuity of our profit functions (Gliksberg 1952; Dasgupta and Maskin 1986). Despite the absence of guarantees for the existence of pure Nash equilibrium in the general case, the partial characterization of pure equilibria is possible for a broad range of problem parameters:

Proposition 11. Define $\rho_{1}=\frac{\pi_{\mathrm{o}} \theta}{\pi_{\mathrm{n}}}$ and $\rho_{2}=\frac{\pi_{\mathrm{o}}(1-\theta)}{\pi_{\mathrm{n}}}$.

a) Suppose that $\rho_{1} \leq 1$ and $\rho_{2} \leq 1$. Then, $t_{1}^{*}=t_{2}^{*}=0$.

b) Suppose that $\beta<\frac{p_{\mathrm{n}}\left(q_{\mathrm{n}}+3 p_{\mathrm{n}}\right)}{q_{\mathrm{n}}+2 p_{\mathrm{n}}}$ and $\rho_{1} \geq \frac{1}{1-\frac{\beta\left(q_{\mathrm{n}}+2 p_{\mathrm{n}}\right)}{p_{\mathrm{n}}\left(q_{n}+3 p_{\mathrm{n}}\right)}}$, $\rho_{2} \geq \frac{1}{1-\frac{\beta\left(q_{\mathrm{n}}+2 p_{\mathrm{n}}\right)}{p_{\mathrm{n}}\left(q_{\mathrm{n}}+3 p_{\mathrm{n}}\right)}}$. Then, $t_{1}^{*}=t_{2}^{*}=t_{\max }$.

c) Suppose that $\beta<\frac{p_{\mathrm{n}}\left(q_{\mathrm{n}}+3 p_{\mathrm{n}}\right)}{p_{\mathrm{n}}+2 p_{\mathrm{n}}}$ and $\rho_{1} \geq \frac{1}{1-\frac{\beta\left(q_{\mathrm{n}}+2 p_{\mathrm{n}}\right)}{p_{\mathrm{n}}\left(q_{n}+3 p_{\mathrm{n}}\right)}}$, $\rho_{2} \leq 1$. Then, $t_{1}^{*}=t_{\max }$ and $t_{2}^{*}=0$. 


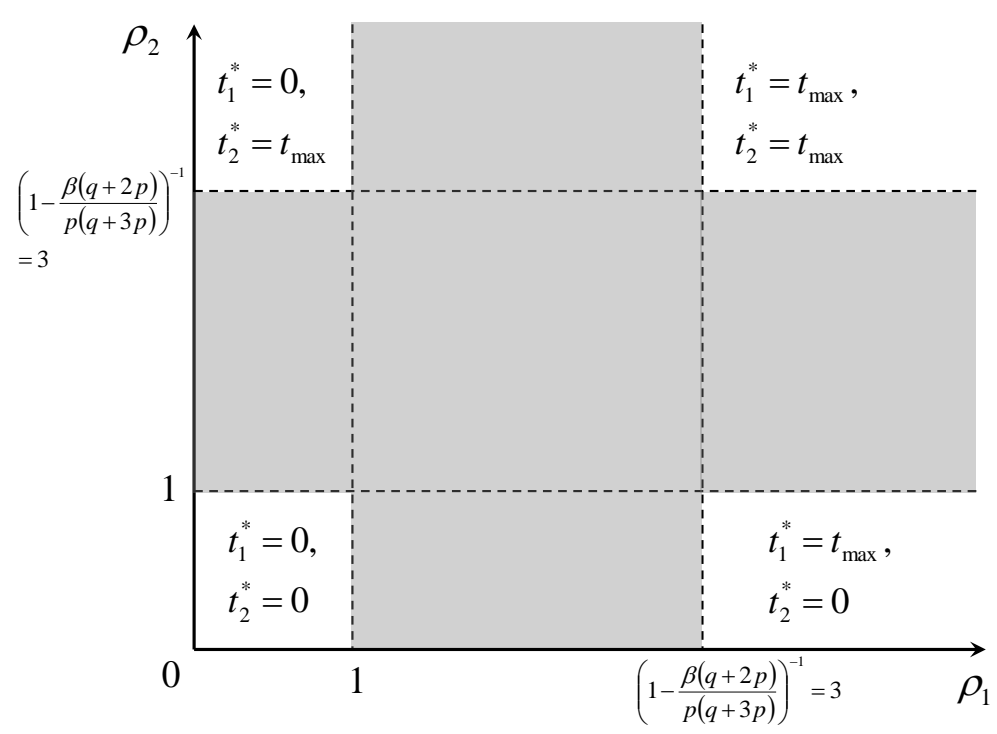

Figure 5 Nash equilibria with respect to new service model adoption times $\left(\beta=p_{n}=1.3 \times 10^{-4}, q_{n}=0\right)$.

The statements of Proposition 11 are illustrated on Figure 5 for the case of $2 \beta=p_{\mathrm{n}}=1.3 \times 10^{-4}$, $q_{\mathrm{n}}=0$. For this parameter combination, $\left(1-\frac{\beta\left(q_{\mathrm{n}}+2 p_{\mathrm{n}}\right)}{p_{\mathrm{n}}\left(q_{\mathrm{n}}+3 p_{\mathrm{n}}\right)}\right)^{-1}=1.5$, and Proposition 11 identifies the Nash equilibria for a wide range of problem settings. Clearly, in the cases not covered by the statements of Proposition 11, the existence of a pure-strategy Nash equilibrium and the values of the matching adoption response times have to be established through the numerical evaluation of the profit functions of competing firms. Note that the "grey areas" of the parameter space not covered by Proposition 11 shrink as the time discount factor $\beta$ goes down, reflecting the growing attractiveness of the "delay as much as you can" option for both incumbents. In particular, in the limit of $\beta \rightarrow 0$, Proposition 11 completely describes the adoption behavior of both incumbents for any combination of problem parameters. Table 3 provides a detailed illustration of this limit. Note that the types of possible adoption equilibria in this case are determined by the relative discount offered to customers switching to the new business model, $\frac{\pi_{\mathrm{n}}}{\pi_{\mathrm{o}}}$. In particular, in the "deep discount" case $\left(\frac{\pi_{\mathrm{n}}}{\pi_{\mathrm{o}}} \leq 0.5\right)$ the major incumbent always delays its adoption as much as possible, while the minor incumbent uses the "delay as much as possible" approach when the initial customer base of the major incumbent is not too high $\left(\theta \leq 1-\frac{\pi_{\mathrm{n}}}{\pi_{\mathrm{o}}}\right)$, and the "adopt now" approach otherwise. On the other hand, when the new model does not offer a significant discount $\left(\frac{\pi_{\mathrm{n}}}{\pi_{\mathrm{o}}}>0.5\right)$, the minor 


\begin{tabular}{|c|c|c|}
\hline & \multicolumn{2}{|c|}{ Profit Discount, $\frac{\pi_{\mathrm{n}}}{\pi_{\mathrm{o}}}$} \\
\hline Market Dominance, $\theta$ & Moderate $\left(\frac{\pi_{\mathrm{n}}}{\pi_{\mathrm{o}}}>0.5\right)$ & $\operatorname{Deep}\left(\frac{\pi_{\mathrm{n}}}{\pi_{\mathrm{o}}} \leq 0.5\right)$ \\
\hline Moderate $\left(\theta \leq \max \left(\frac{\pi_{\mathrm{n}}}{\pi_{\mathrm{o}}}, 1-\frac{\pi_{\mathrm{n}}}{\pi_{\mathrm{o}}}\right)\right)$ & Major Incumbent- "Now" & Major Incumbent- "Delay" \\
\hline Strong $\left(\theta>\max \left(\frac{\pi_{\mathrm{n}}}{\pi_{\mathrm{o}}}, 1-\frac{\pi_{\mathrm{n}}}{\pi_{\mathrm{o}}}\right)\right)$ & Minor Incumbent- "Now" & Minor Incumbent- "Delay" \\
\hline & Minor Incumbent- "Delay" & Major Incumbent- "Delay" \\
\hline
\end{tabular}

Table 3 Adoption Nash-equilibrium strategies in the absence of time discounting.

incumbent adopts the new model immediately, while the policy of the major incumbent changes from "adopt now" in settings where its market dominance is limited $\left(\theta \leq \frac{\pi_{\mathrm{n}}}{\pi_{\mathrm{o}}}\right)$ to "delay as much as possible" when it is pronounced $\left(\theta>\frac{\pi_{\mathrm{n}}}{\pi_{\mathrm{o}}}\right)$. The results of Proposition 11 indicate that in the business settings where the customer base values of competing firms follow Bass-like dynamics introduced in Section 2, major incumbents (dominant firms) are prone to delays in innovation. An intuitive, simple nature of the equilibria described in Table 3 offers competitors an appealing recipe for building their adoption strategies in settings where the time discounting factors are negligible.

\section{Nash Equilibria Adoption Times in the FX Market: A Numerical Study}

The analytical results presented in Section 4 relate to the special setting where the innovation is guaranteed to be successful, does not affect the overall market potential, and where the customer base dynamics exhibits negligible intra-generational churn. The FX market example introduced in Section 3 describes an environment without the market expansion upon the introduction of new service model, no intra-generational churn in the diffusion dynamics of the new service model, but where the intra-generational churn in the dynamics of the old service model is prominent. For such a setting we have conducted numerical tests of the Nash equilibria in the adoption times for the new service model. In particular, we have used the diffusion parameters and the market size estimates reported in Table 2 and varied the parameters that describe the incumbents' beliefs about the risk associated with the new service model, i.e., their beliefs regarding the probability and the time of its potential "failure". 
Note that, in addition to the diffusion, market size, and risk parameters, the Nash equilibrium adoption times are determined by the ratio $\frac{\pi_{\mathrm{n}}}{\pi_{\mathrm{o}}}$ of the profit margins associated with the old and the new service model, as well as the time discount factor $\beta$. We have estimated the profit margin associated with the the traditional, phone-based, business model, $\pi_{\mathrm{o}}$, to be $11 \%$ by averaging the profit margins for 1998-2000 from the 10-k reports for the Bank of New York and Merrill Lynch (Bank of New York Mellon 2001, Merrill Lynch 2002). The interviews we have conducted with senior executives in the industry have confirmed a significant reduction in the profit margin upon switching to the online trading model, with $\pi_{\mathrm{n}}$ being about $5 \%$. In our numerical tests, we have used these estimates for the $\pi_{\mathrm{o}}$ and $\pi_{\mathrm{n}}$, respectively, resulting in the ratio of $\frac{\pi_{\mathrm{n}}}{\pi_{\mathrm{o}}}=45.45 \%$. For the time-discounting factor, we have used $\beta=0.01$ (per month), corresponding to $12 \%$ annual discounting rate.

In calculating the Nash equilibria adoption times, we have assumed that each incumbent commits to entering at a particular (integer) month value between the launch of the new model and the finite horizon of $t_{\max }=30$ months. We have selected the moderately high finite horizon value to ensure that the numerical search for the Nash equilibria can be accomplished in reasonable time. For each combination of problem parameters reported below, the Nash equilibrium in incumbent adoption times exists and is unique when the incumbents' choices are limited to the set of integers between 0 and $t_{\max }$.

Figure 6 reports the Nash equilibrium incumbent adoption times as functions of the "failure" probability $P_{F}$ for two fixed values of the "failure" time, $T_{F}=15$ and $T_{F}=60$. We have selected these two values to illustrate the difference in incumbents' responses to the failure that occurs "early" $\left(T_{F}<t_{\max }\right)$ and to the one that occurs "late" $\left(T_{F}>t_{\max }\right)$. The adoption-time Nash equilibria described in Figure 6 reflect a rather interesting competitive dynamics among the incumbents. Note the "discontinuity" in the Nash equilibrium pattern observed in Figure 6a that describes the case of an early failure $\left(T_{F}=15<t_{\max }\right)$. In this setting, when $P_{F}=1$, i.e., when the failure is certain, both incumbents react to such early and certain failure by abandoning the adoption of 


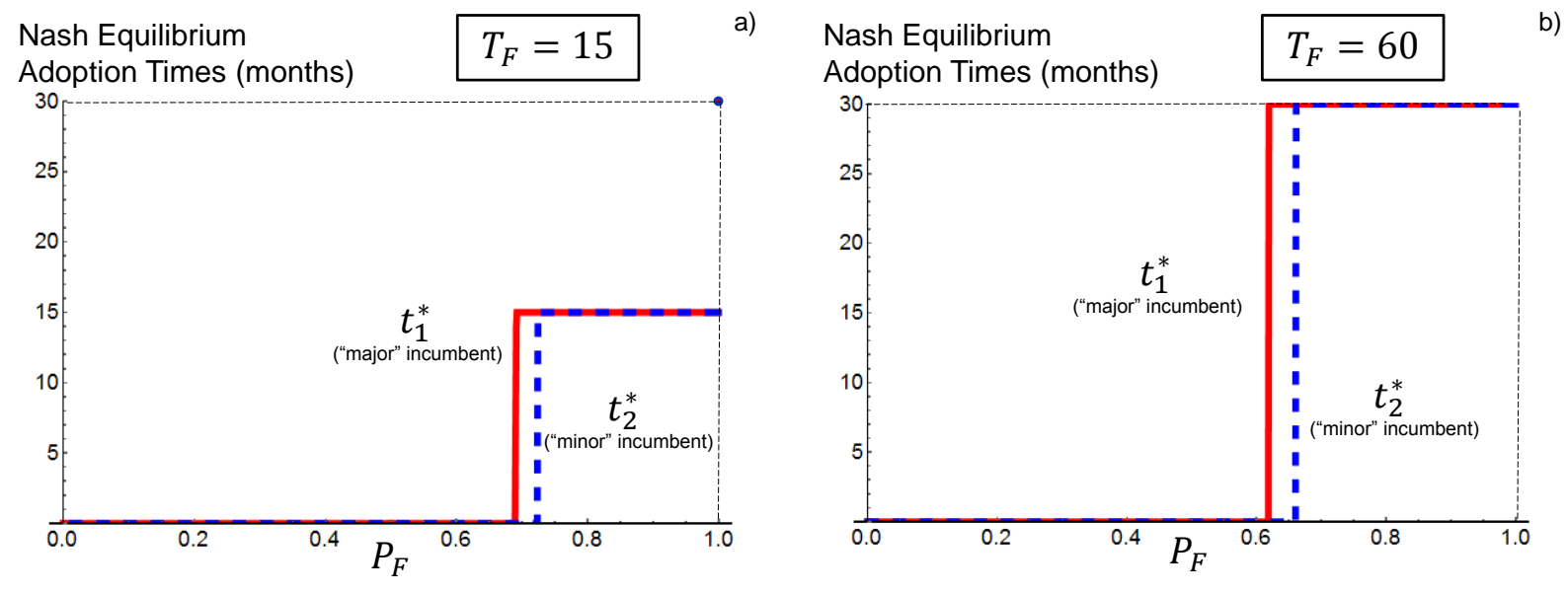

Figure 6: The Nash equilibrium adoption times for the two incumbents in the FX example as functions of the failure probability $P_{F}$ for the determinsitic failure times $T_{F}=15$ (a), and $T_{F}=60$ (b).

the innovation. However, as soon as there appears to be a finite probability for the innovation to survive, both incumbents immediately hedge by committing to an adoption at the potential failure time $T_{F}=15$. When the failure probability is reduced further, there emerges a range of probability values such that the "minor" incumbent favors immediate adoption, while the "major" incumbent commits to a delayed adoption at $T_{F}=15$. Finally, as the failure becomes even less likely, both incumbents choose immediate adoption. In our numerical tests, a similar Nash equilibrium pattern is observed for any fixed $0<T_{F}<t_{\max }$.

Figure 6b illustrates a corresponding monotonicity in the incumbents' responses when failure, if it occurs, happens fairly late, beyond the allowable horizon of incumbents' responses. In this case, incumbents cannot commit to adopting at $T_{F}$, and the "adopt now" response directly follows "never adopt" response as the survival of the new service model becomes increasingly likely.

We observed a similar response pattern for all values of $T_{F}>t_{\max }$, with the region of "never adopt" response shrinking as the failure time becomes more distant. In particular, for $T_{F} \geq 115$, both incumbents choose "adopt now" response irrespective of the probability of failure. For such high values of $T_{F}$ incumbents behave as if failure does not occur at all and immediately move to protect their profit streams. 


\section{Nash Equilibrium Adoption Times (months)}

$$
P_{F}=0.8
$$

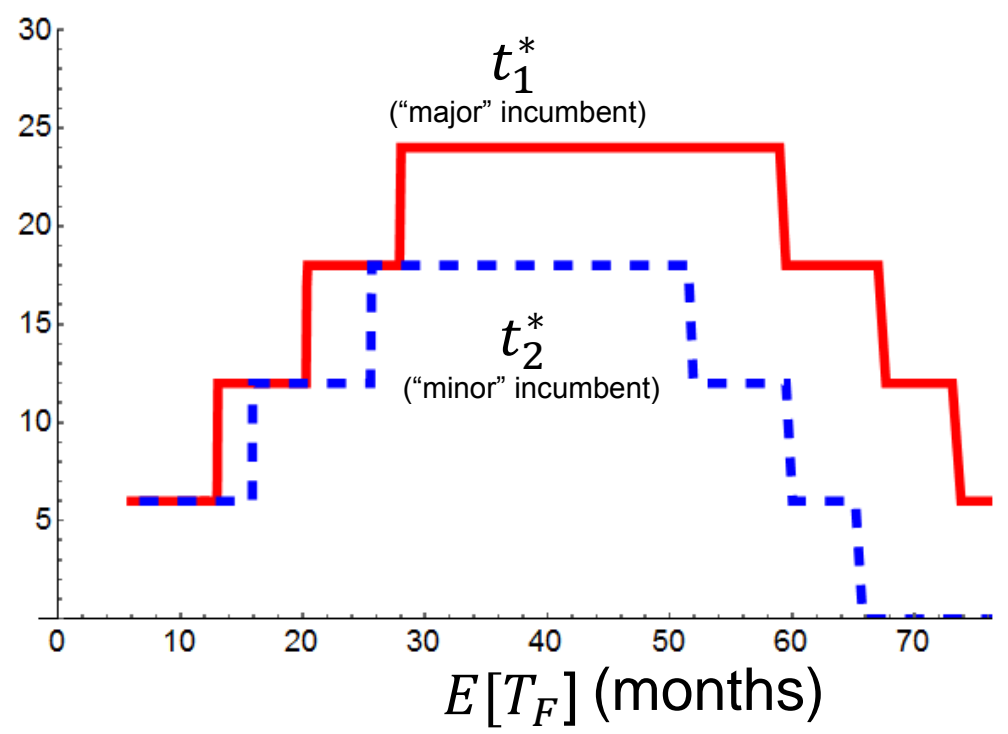

Figure 7: The Nash equilibrium adoption times for the two incumbents in the FX example as functions of the expected failure time $E\left[T_{F}\right]$ for the geometric distribution of the failure time period $\left(P_{F}=0.8\right)$.

Figure 6 illustrates the properties of the Nash equilibria in settings where the failure of the new model, if it occurs, happens at a given time period. A more interesting setting is shown in Figure 7 , where the time-to-failure, if it occurs, follows the variant of geometric distribution, i.e.,

$$
P\left(T_{F}=k\right)=p(1-p)^{k}, k=6,12,18,24, \ldots
$$

Note that, under the probability distribution (73), the failure of the new model can occur at 6month increments (i.e., at $t=6,12,18,24, \ldots$, months), and is "memoryless". The latter feature of this distribution provides an appropriate match to the open-loop nature of the incumbents' decision process on when to adopt an innovation. In other words, under the geometric time-tofailure distribution, the incumbents cannot improve their decision making process by adopting a dynamic, "closed-loop" policy that would entail making an adoption decision based on time elapsed since the introduction of the new service model. Figure 7 shows the Nash equilibrium adoption times for the incumbents as a function of the expected time-to-failure for the setting where the failure is quite likely $\left(P_{F}=0.8\right)$. This high value of the failure probability reflects an 
important outcome we have observed: both incumbents choose "immediate" adoption (i.e, the Nash equilibrium is $t_{1}^{*}=t_{2}^{*}=0$ ) for all values of $E\left[T_{F}\right]$ as long as the failure probability $P_{F}$ is low. Hence, in Figure 7 we report the results for the value of $P_{F}$ that results, at least for some values of $T_{F}$, in "delayed-adoption" Nash equilibria.

There are three key observations that we can make based on the results shown in Figure 7.

First, the insight outlined in Proposition 10 continues to hold in a more general setting of the FX market example. In particular, the customer base dominance in terms of the old model translates into "inertia" in adopting the new service model: the "major" incumbent never leads in terms of adoption of the new service model, and either enters simultaneously with the "minor" incumbent, or lags behind it.

Second, in the FX market setting, incumbents must estimate the failure of the new model to be quite likely to abandon the "immediate adoption" equilibrium. In particular, for $P_{F}=0.8$ (i.e., for the setting where the failure of the new model is likely, but not certain), we observe an emergence of the "delay equilibria" when the expected failure time is neither too small nor too large.

Third, the equilibrium adoption responses of the incumbents exhibit a clear non-monotone pattern as $T_{F}$ grows. In particular, when the failure is expected in a "near future", the marginal impact of failure delays on the adoption delays is positive, as the incumbents react by increasing the protection of their old customer bases and delaying their adoption decisions. However, when the $T_{F}$ reaches a particular threshold, the failure of the new model, even if it happens with high probability, is late enough to lose most of its impact. From the incumbents' perspective, the situation begins to look more and more like the setting where the new model succeeds, and this forces the incumbents to reverse their assessments and lean towards the "immediate" adoptions.

As mentioned in Section 3, in the FX market example the "minor" incumbent has adopted the new model at $t_{2}=13$, and the "major" incumbent - at $t_{1}=14$ months. These adoption times are close to the Nash equilibria in the setting where incumbents assign a high probability of failure to the new model, expecting the failure to occur at around a two-year mark. 


\section{Conclusions and Managerial Implications}

The question of when an incumbent firm should cannibalize the customer base from an existing service model as a result of adopting a new service model introduced by a "new entrant" firm is of major importance in a number of business settings. In particular, is market dominance of the incumbent a hindrance or an incentive for an early adoption? The theoretical and empirical literature on this issue provides conflicting answers. Our paper addresses the limitations of the existing literature and provides a detailed analytical account of customer adoption dynamics and how it might affect firms' profits and influence innovation adoption decisions. Our approach to describing customer base trajectories extends the classical Bass adoption dynamics to the setting where two incumbent firms compete with an entrant proposing a new service model.

In our analysis, we model a setting that includes the market expansion upon the introduction of the new service model, as well as the intra-generational customer churn. We derive closed-form expressions for the customer base trajectories of competing firms in two special settings. In the first setting, we assume that the introduction of the innovation leads to an expansion of the market potential and the diffusion dynamics includes intra-generational customer churn. In the second setting, we assume that there is no intra-generational churn and the market potential remains unchanged upon the introduction of innovation, but there are brand awareness effects whereby the incumbents enjoy a larger customer imitation effect compared to the new entrant. For the latter setting, we analyze the optimal adoption response decisions and the Nash equilibria adoption times. Our results show that under the Bass-like competitive dynamics, the market dominance translates into lagged response to innovation as long as the size of the pre-innovation customer base is the main distinguishing feature between the incumbents.

One important aspect of our work is the analysis of potential risks associated with the new service model. In particular, we analyze, analytically, the diffusion dynamics of the two incumbents following the new model failure, and, numerically, the Nash equilibrium adoption decisions for the incumbents that share estimates for the probability of failure of the new service model and the 
distribution of time at which such failure may occur. For the latter investigation, we use the actual trading volume data from the FX markets to identify the best-fit diffusion dynamics parameters and to study the effects of the likelihood and the imminence of the new model failure on the responses of the incumbents. Our results, in particular, help to quantify an important connection between the delays in the adoption of the new business model and the incumbents' perception of the expected duration for the new model to remain on the market.

The model we propose and the analysis we conduct have several managerial implications. First, for managers it is important to distinguish between innovation and imitation effects of not only one's own firm but also those of competitors when introducing new products, services and business models. Managers have increasingly come to realize the importance of market research and ways to affect interpersonal communications, such as word of mouth, especially in a world where social media is increasingly playing a major role. However, as a result of easier data availability, firms often focus on measuring and understanding their own innovation and imitation effects as part of marketing communications but less on those of their competitors. In particular, the brand awareness effect often comes through the imitation effect, whereby communication about the new adoption experience from an existing customer of the firm might have a stronger effect on the willingness of other customers to switch to the innovation introduced by the firm, as compared to the imitation effect coming from customers of a new entrant. Managers need to evaluate fully the relative influence of these two effects and use this knowledge for prediction and planning on when to adopt an innovation.

Second, it is important for managers to have reliable estimates of the churn rate for existing services. Often, customers switch between incumbent firms that provide an old service even when new one is launched. Some of these customers may churn and choose a different service provider due to selection or quality-related reasons. This customer switching pattern creates intra-generational churn, which is an important factor influencing customer base trajectories and profits for the competing firms. 
Third, as the introduction of innovation can result in market expansion for both the existing service model and the new service model, managers need to understand how the various sources of interpersonal communication influence such market expansion. While a number of previous studies on the subject of innovation have looked at the implications of a new entrant on the incumbent's incentive to innovate (Schumpeter 1942; Ghemawat 1991; Chandy and Tellis 2000), we adopt a more nuanced view of how the effect of the new entrant works through the incentives of other incumbent firms. In our analysis, the adoption decision of other incumbent firms is just as important as that of the new entrant. Managers can use our model to assess changes taking place in the diffusion process because of adoption by another incumbent firm and to analyze possible "what if" scenarios to understand the impact of their actions on the terminal sizes of their customer bases and profitability.

In the present paper we focused on the diffusion dynamics and assumed a pre-set marketing-mix environment and a symmetry in the incumbents diffusion parameters. One promising extension to our work is to model the influence of dynamically set prices on the resulting adoption-time Nash equilibria. Another avenue for future research relates to the "now" or "never" innovation adoption prescription described by Wilson and Norton (1989) and refined by Mahajan and Muller (1996). In particular, whether such a policy remains an optimal response for competing firms in the presence of intra-generational churn and post-innovation market expansion is an important question for further investigation. Finally, the important question we left outside of the scope of our present investigation is how robust the inertia in adopting the innovation is in settings with asymmetric incumbents. We anticipate that a reversal of such market entry order can only be observed in settings where the pre-innovation customer base asymmetry is complemented by a substantial additional asymmetry in the incumbents' diffusion parameters. Identifying such settings is an important future step in characterizing competitive market-entry equilibria.

In our analysis we have assumed that the competing firms have ample capacity to handle the consumer demand. It is possible, however, especially in the case of the new entrant, that the 
limited ability to accommodate growing demand may slow down the process of innovation diffusion (Balakrishnan and Pathak 2014). Extending our analysis to constrained-capacity settings is a promising direction for future research.

\section{Acknowledgments}

We would like to thank Svetlana Rieb for her assistance on the data collection for the empirical example. We would also like to thank Sriya Iyer, Zhan Phang, Eden Yin and seminar participants at the Cambridge Judge Business School for their comments and suggestions on earlier versions of the manuscript.

\section{References}

Aboulnasr, K., O. Narashimhan, E. Blair, R. Chandy. 2008. Competitive Response to Radical Product Innovations. Journal of Marketing 72 94-110.

Balakrishnan, P.V., S. Pathak. 2014. Impeding the Juggernaut of Innovation Diffusion: A Production-Constrained Model. Production and Operations Management 23(7) 1183-1197.

Bank of New York-Mellon. 2001. 10-k Report 2000, New York: BNYM.

Bass, F.M. 1969. A New Product Growth Model for Consumer Durables. Management Science 16(5) 215-227.

Benoit, J.-P. 1985. Innovation and Imitation in a Duopoly. Rev. Econ. Studies 52(1), 99-106.

Bhargava, H.K., B.C. Kim, D. Sun. 2013. Commercialization of Platform Technologies: Launch Timing and Versioning Strategy. Production and Operations Management 22(6) 1374-1388.

Chandy, R.K., G.J. Tellis. 2000. The Incumbent's Curse? Incumbency, Size and Radical Product Innovation. Journal of Marketing 64 1-17.

Dasgupta, P., E. Maskin. 1986. The Existence of Equilibrium Points in Discontinuous Economic Games, I and II. Review of Economic Studies 53(1) 1-41.

Doraszelski, U. 2004. Innovations, Improvements and the Optimal Timing of New Technologies. $J$. Econ. Dyn. and Control 28 1461-1480.

Euromoney 2000. FX Poll, May 2000. 
Frattini, F., C. Dell'Era, A. Rangone. 2013. Launch Decisions and the Early Market Survival of Innovations: An Empirical Analysis of the Italian Mobile Value-Added Services (VAS) Industry. $J$. Prod. Innov. Management 30 174-187.

Ghemawat, P. 1991. Market Incumbency and Technological Inertia. Marketing Science 10(2) 161171.

Gilbert, R.J., M. Lieberman. 1987. Investment and Coordination in Oligopolistic Industries. Rand J. Econ. 18(1) 17-33.

Gliksberg, I.L. 1952. A Further Generalization of the Kakutani Fixed Point Theorem with Application to Nash Equilibrium Points. Proceedings of the American Mathematical Society 38(1)170-174. Golder, P.N., G.J. Tellis. 1993. Pioneer Advantage: Marketing Logic or Marketing Legend. Journal of Marketing Research 30 (May) 158-170.

Gupta, S., D.R. Lehmann, J.A. Stuart. 2004. Valuing Customers. Journal of Marketing Research 41(1) $7-18$.

Guseo, R., C. Mortarino. 2010. Correction to the paper "Optimal product launch times in a duopoly: balancing life-cycle revenues with product cost". Oper. Res. 58 1522-1523.

Guseo, R., C. Mortarino. 2012. Sequential market entries and competition modelling in multiinnovation diffusions. Eur. J. Oper. Res. 216 658-667.

Guseo, R., C. Mortarino. 2014. Within-brand and cross-brand word-of-mouth for sequential multiinnovation diffusions. IMA J. Manag. Math. 25 287-311.

Guseo, R., C. Mortarino. 2015. Modeling competition between two pharmaceutical drugs using innovation diffusion models. Annals of Applied Statistics 9 2073-2089.

Haunschild, P.R., A.S. Miner. 1997. Modes of Inter-Organizational Imitation: The Effects of Outcome Salience and Uncertainty. Admin. Sci. Quarterly 42 472-500.

Jensen, R. 1988. Information Cost and Innovation Adoption Policies. Management Science 34(2) 230-239.

Joshi, Y.V., D. J. Reibstein, Z. J. Zhang. 2009. Optimal Entry Timing in Markets with Social Influence. Management Science, 55(6) 926-939. 
Kalish, S., V. Mahajan, E. Muller. 1995. Waterfall and Sprinkler New Product Strategies in Competitive Global Markets. International Journal of Research in Marketing 12(2) 105-109.

King, A.A., C.L. Tucci. 2002. Incumbent Entry into New Market Niches: The Role of Experience and Managerial Choice in the Creation of Dynamic Capabilities. Management Sci., 48(2) 171-186. Krishnan, T.V., F.M. Bass, V. Kumar. 2000. Impact of a Late Entrant on the Diffusion of a New Product/Service. Journal of Marketing Research 37 269-278.

Libai, B., E. Muller, R. Peres. 2009a. The Role of Within-Brand and Cross-Brand Communications in Competitive Growth. Journal of Marketing 73(May) 19-34.

Libai, B., E. Muller, R. Peres. 2009b. The Diffusion of Services. Journal of Marketing Research 46 (April), 163-175.

Lilien, G.L., A. Rangaswamy, C. Van den Bulte. 2000. Diffusion Models: Managerial Applications and Software in New-Product Diffusion Models, ed. by Vijay Mahajan, Eitan Muller, and Yoram Wind, New York: Springer.

Mahajan, V., E. Muller. 1996. Timing, Diffusion, and Substitution of Successive Generations of Technological Innovations: The IBM Mainframe Case. Technological Forecasting and Social Change 51(2) 109-132.

Mahajan, V., S. Sharma, R.D. Buzzell. 1993. Assessing the Impact of Competitive Entry on Market Expansion and Incumbent Sales. Journal of Marketing 57(3) 39-52.

Mamer, J.W., K.F. McCardle. 1987. Uncertainty, Competition and the Adoption of New Technology. Management Science 33(2) 161-177.

McCardle, K.F. 1984. Information Acquisition and the Adoption of New Technology. Management Sci. 31(11) 1372-1389.

Merrill Lynch. 2002. 10-k Report 2001, New York: Merrill Lynch.

Peres, R., E. Muller, V. Mahajan. 2010. Innovation Diffusion and New Product Growth Models: A Critical Review and Research Directions. International Journal of Research in Marketing $\mathbf{2 7}$ 91-106. 
Rangaswamy, A., S. Gupta. 1999. Innovation Adoption and Diffusion in the Digital Environment: Some Research Opportunities. Working paper 02-1999, eBusiness Research Center, Pennsylvania State University, PA.

Reuters News. 2001. Daily FX on-line trade on Currenex tops $\$ 1$ bln. July 13, 2001.

Ringel, M., A. Taylor, H. Zablit. 2015. The Rising Need for Innovation Speed. The Boston Consulting Group.

Rogers, E. 1962. Diffusion of innovations (1st ed.) New York: Free Press of Glencoe.

Savin, S., C. Terwiesch. 2005. Optimal Product Launch Times in a Duopoly: Balancing Life-Cycle Revenues with Product Cost. Operations Research 53(1) 26-47.

Schumpeter, J.A. 1942. Capitalism, Socialism, and Democracy. Harper and Brothers, New York, NY.

Shankar, V., G.S. Carpenter, L. Krishnamurthy. 1998. Late Mover Advantage: How Innovative Late Entrants Outsell Pioneers. Journal of Marketing Research 35(1) 54-70.

Sorescu, A.B., R.K. Chandy, J. Prabhu. 2003. Sources and Financial Consequences of Radical Innovation: Insights from Pharmaceuticals. Journal of Marketing 66(4) 82-102.

Wilson, L.O., J.A. Norton. 1989. Optimal Entry Timing for a Product Line Extension. Marketing Science 8(1) 1-17. 


\section{Appendices for "When to Adopt a Service Innovation: Nash Equilibria in a Competitive Diffusion Framework"}

\section{Appendix A: Proofs of Analytical Results}

\section{Proof of Proposition 1}

The Bass-like form of $m_{3}^{\mathrm{n}}(t)(4)$ is obtained by directly integrating (3) with the initial condition $m_{3}^{\mathrm{n}}(0)=0$. Let us denote $m^{\text {tot }}(t)=m_{1}^{\mathrm{o}}(t)+m_{2}^{\mathrm{o}}(t)+m_{3}^{\mathrm{n}}(t)$. Then, adding equations (1)-(3), we get

$$
\frac{d m^{\mathrm{tot}}}{d t}=\left(2 p_{\mathrm{o}}+p_{\mathrm{n}}+q_{\mathrm{o}} \frac{m^{\mathrm{tot}}}{\mathcal{M}}+\left(\frac{q_{\mathrm{n}}-q_{\mathrm{o}}}{\mathcal{M}}\right) m_{3}^{\mathrm{n}}\right)\left(\mathcal{M}-m^{\mathrm{tot}}\right)
$$

Denoting $X(t)=\frac{\mathcal{M}}{\mathcal{M}-m^{\text {tot }}}$, we can rewrite (A1) as $\frac{d X}{d t}-f(t) X=-q_{\mathrm{o}}$ with $f(t)=2 p_{\mathrm{o}}+p_{\mathrm{n}}+q_{\mathrm{o}}+$ $\left(\frac{q_{\mathrm{n}}-q_{\mathrm{o}}}{\mathcal{M}}\right) m_{3}^{\mathrm{n}}(t)$ and the initial condition $X(0)=\frac{\mathcal{M}}{\mathcal{M}-m-M}$. The solution to the latter equation with this initial condition is given by

$$
X(t)=\left(\frac{\mathcal{M}}{\mathcal{M}-m-M}-q_{\mathrm{o}} \int_{0}^{t} d \tau \exp \left(-\int_{0}^{\tau} f(u) d u\right)\right) \exp \left(\int_{0}^{t} f(\tau) d \tau\right) .
$$

Note that $\int_{0}^{t} f(\tau) d \tau$

$$
=\int_{0}^{t}\left(2 p_{\mathrm{o}}+p_{\mathrm{n}}+q_{\mathrm{o}}+\left(\frac{q_{\mathrm{n}}-q_{\mathrm{o}}}{\mathcal{M}}\right) m_{3}^{\mathrm{n}}(\tau)\right) d \tau=\left(2 p_{\mathrm{o}}+p_{\mathrm{n}}+q_{\mathrm{n}}\right) t+\left(\frac{q_{\mathrm{n}}-q_{\mathrm{o}}}{q_{\mathrm{n}}}\right) \log \left(\frac{q_{\mathrm{n}} e^{-\left(q_{\mathrm{n}}+p_{\mathrm{n}}\right) t}+p_{\mathrm{n}}}{q_{\mathrm{n}}+p_{\mathrm{n}}}\right),
$$

and $\int_{0}^{t} d \tau \exp \left(-\int_{0}^{\tau} f(u) d u\right)$

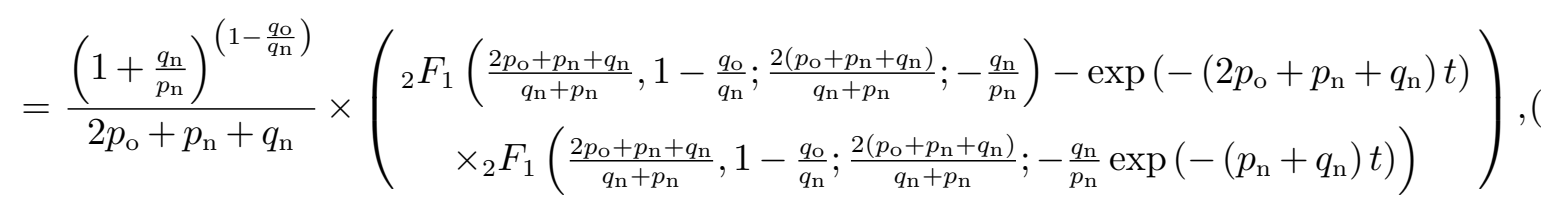

where ${ }_{2} F_{1}(a, b ; c ; x)=\sum_{n=0}^{+\infty} \frac{(a)_{n}(b)_{n}}{n !(c)_{n}} x^{n}$ is the hypergeometric function. Using (5), we get

$$
X(t)=\left(\frac{\mathcal{M}}{\mathcal{M}-m-M}-\frac{\widehat{F}_{I}\left(p_{\mathrm{o}}, p_{\mathrm{n}}, q_{\mathrm{o}}, q_{\mathrm{n}}, t\right) q_{\mathrm{o}}\left(1+\frac{q_{\mathrm{n}}}{p_{\mathrm{n}}}\right)^{\left(1-\frac{q_{\mathrm{o}}}{q_{\mathrm{n}}}\right)}}{2 p_{\mathrm{o}}+p_{\mathrm{n}}+q_{\mathrm{n}}}\right)\left(\frac{q_{\mathrm{n}}+p_{\mathrm{n}} e^{\left(q_{\mathrm{n}}+p_{\mathrm{n}}\right) t}}{\left(q_{\mathrm{n}}+p_{\mathrm{n}}\right) e^{\left(q_{\mathrm{n}}+p_{\mathrm{n}}\right) t}}\right)^{\left(\frac{q_{\mathrm{n}}-q_{\mathrm{o}}}{q_{\mathrm{n}}}\right)} e^{\left(\left(2 p_{\mathrm{o}}+p_{\mathrm{n}}+q_{\mathrm{n}}\right) t\right)},
$$


and

$$
m^{\text {tot }}(t)=\mathcal{M}\left(1-\frac{\exp \left(-\left(2 p_{\mathrm{o}}+p_{\mathrm{n}}+q_{\mathrm{n}}\right) t\right)\left(\frac{q_{\mathrm{n}}+p_{\mathrm{n}} e^{\left(q_{\mathrm{n}}+p_{\mathrm{n}}\right) t}}{\left(q_{\mathrm{n}}+p_{\mathrm{n}}\right) e^{\left(q_{\mathrm{n}}+p_{\mathrm{n}}\right) t}}\right)^{-\left(\frac{q_{\mathrm{n}}-q_{\mathrm{o}}}{q_{\mathrm{n}}}\right)}}{\left(\frac{\mathcal{M}}{\mathcal{M}-m-M}-\frac{q_{\mathrm{o}}\left(1+\frac{q_{\mathrm{n}}}{p_{\mathrm{n}}}\right)\left(1-\frac{q_{\mathrm{o}}}{q_{\mathrm{n}}}\right)}{2 p_{\mathrm{o}}+p_{\mathrm{n}}+q_{\mathrm{n}}} \widehat{F}_{I}\left(p_{\mathrm{o}}, p_{\mathrm{n}}, q_{\mathrm{o}}, q_{\mathrm{n}}, t\right)\right)}\right) .
$$

Now, using (7), defining $m^{-}(t)=m_{1}^{\circ}(t)-m_{2}^{\circ}(t)$ and subtracting (2) from (1), we get

$$
\frac{d m^{-}(t)}{d t}=\left(-2 p_{\mathrm{oc}}-p_{\mathrm{n}}+q_{\mathrm{o}}-q_{\mathrm{o}} \widehat{G}_{I}\left(p_{\mathrm{o}}, p_{\mathrm{n}}, q_{\mathrm{o}}, q_{\mathrm{n}}, t\right)-\frac{q_{\mathrm{n}}}{\mathcal{M}} m_{3}^{\mathrm{n}}\right) m^{-}(t),
$$

with the initial condition $m^{-}(0)=M-m$. The solution to (A7) with this initial condition is

$$
m^{-}(t)=\frac{(M-m)\left(q_{\mathrm{n}}+p_{\mathrm{n}}\right) \exp \left(\left(q_{\mathrm{o}}-2 p_{\mathrm{oc}}\right) t\right)}{q_{\mathrm{n}}+p_{\mathrm{n}} e^{\left(q_{\mathrm{n}}+p_{\mathrm{n}}\right) t}} e^{\left(-q_{\mathrm{o}} \int_{0}^{t} \widehat{G}_{I}\left(p_{\mathrm{o}}, p_{\mathrm{n}}, q_{\mathrm{o}}, q_{\mathrm{n}}, \tau\right) d \tau\right)}=\widehat{H}_{I}\left(p_{\mathrm{o}}, p_{\mathrm{oc}}, p_{\mathrm{n}}, q_{\mathrm{o}}, q_{\mathrm{n}}, t\right) .
$$

Given that $m_{1}^{\mathrm{o}}(t)+m_{2}^{\mathrm{o}}(t)=m^{\mathrm{tot}}(t)-m_{3}^{\mathrm{n}}(t)=\mathcal{M} \widehat{G}\left(p_{\mathrm{o}}, p_{\mathrm{n}}, q_{\mathrm{o}}, q_{\mathrm{n}}, t\right)-m_{3}^{\mathrm{n}}(t)$, we get $(9)$.

\section{Proof of Corollary 1.1}

The customer base of the new entrant, $m_{3}^{\mathrm{n}}(t)$, remains identical to that in Proposition 1 . Plugging in $q_{\mathrm{o}}=0$ into equation (A1) we get

$$
\frac{d m^{\mathrm{tot}}}{d t}=\left(2 p_{\mathrm{o}}+p_{\mathrm{n}}+\frac{q_{\mathrm{n}}}{\mathcal{M}} m_{3}^{\mathrm{n}}\right)\left(\mathcal{M}-m^{\mathrm{tot}}\right) .
$$

with the initial condition $m^{\text {tot }}(0)=m+M$. The solution to (A9) with this initial condition is

$$
m^{\mathrm{tot}}(t)=\mathcal{M}-\frac{(\mathcal{M}-m-M)\left(q_{\mathrm{n}}+p_{\mathrm{n}}\right) e^{-2 p_{\mathrm{o}} t}}{q_{\mathrm{n}}+p_{\mathrm{n}} e^{\left(q_{\mathrm{n}}+p_{\mathrm{n}}\right) t}} .
$$

Using, $m^{-}(t)=m_{1}^{\circ}(t)-m_{2}^{\circ}(t)$ and plugging in $q_{\circ}=0$ into (A7), we get $\frac{d m^{-}(t)}{d t}=$ $\left(-2 p_{\mathrm{oc}}-p_{\mathrm{n}}-\frac{q_{\mathrm{n}}}{\mathcal{M}} m_{3}^{\mathrm{n}}\right) m^{-}(t)$, with the initial condition $m^{-}(0)=M-m$. The solution to these is $m^{-}(t)=\frac{(M-m)\left(q_{\mathrm{n}}+p_{\mathrm{n}}\right) e^{-2 p_{\mathrm{oc}} t}}{q_{\mathrm{n}}+p_{\mathrm{n}} e^{\left(q_{\mathrm{n}}+p_{\mathrm{n}}\right) t}}$. Given that $m_{1}^{\mathrm{o}}(t)+m_{2}^{\mathrm{o}}(t)=m^{\text {tot }}(t)-m_{3}^{\mathrm{n}}(t)$, we obtain $(10)$.

\section{Proof of Corollary 1.2}

The Bass-like form of $m_{3}^{\mathrm{n}}(t)$ remains identical to that in Proposition 1. Plugging in the conditions of Corollary 1.2 into (1)-(2), we have

$$
\frac{d m_{1}^{\mathrm{o}}}{d t}=-p_{\mathrm{n}} m_{1}^{\mathrm{o}}-\frac{q_{\mathrm{n}}}{\mathcal{M}} m_{3}^{\mathrm{n}} m_{1}^{\mathrm{o}}, \frac{d m_{2}^{\mathrm{o}}}{d t}=-p_{\mathrm{n}} m_{2}^{\mathrm{o}}-\frac{q_{\mathrm{n}}}{\mathcal{M}} m_{3}^{\mathrm{n}} m_{2}^{\mathrm{o}} .
$$

Dividing the first equation in (A11) by the second one, and integrating the result with with the initial conditions $m_{1}^{\circ}=M$ and $m_{2}^{o}=m$, we obtain $m_{2}^{\circ}(t)=m_{1}^{\circ}(t)\left(\frac{m}{M}\right)$. Combining this with $m_{1}^{\circ}(t)+$ $m_{2}^{\mathrm{o}}(t)+m_{3}^{\mathrm{n}}(t)=\mathcal{M}$, we get $m_{1}^{\mathrm{o}}(t)=\left(\frac{M}{\mathcal{M}}\right)\left(\mathcal{M}-m_{3}^{\mathrm{n}}(t)\right)$ and $m_{2}^{\mathrm{o}}(t)=\left(\frac{m}{\mathcal{M}}\right)\left(\mathcal{M}-m_{3}^{\mathrm{n}}(t)\right)$. 


\section{Proof of Proposition 2}

Assuming $Q_{\mathrm{n}}=q_{\mathrm{n}}$, we denote $n(t)=m_{2}^{\mathrm{n}}(t)+m_{3}^{\mathrm{n}}(t)$. Then, adding (14)-(15), we get

$$
\frac{d n}{d t}=\left(2 p_{\mathrm{n}}+\frac{q_{\mathrm{n}}}{\mathcal{M}} n\right)(\mathcal{M}-n)
$$

Integrating (A12), with the initial condition $n\left(t_{f}\right)=m_{3}^{\mathrm{n}}\left(t_{f}\right)=\mathcal{M}\left(1-\frac{q_{\mathrm{n}}+p_{\mathrm{n}}}{q_{\mathrm{n}}+p_{\mathrm{n}} e^{\left(q_{\mathrm{n}}+p_{\mathrm{n}}\right) t}}\right)$, we get the Bass-like form of $n(t)=\mathcal{M}\left(1-\frac{2 p_{\mathrm{n}}+q_{\mathrm{n}}}{\alpha e^{\left(2 p_{\mathrm{n}}+q_{\mathrm{n}}\right)\left(t-t_{f}\right)}+q_{\mathrm{n}}}\right)$, where $\alpha=\frac{\left(2 p_{\mathrm{n}}+q_{\mathrm{n}}\right)\left(q_{\mathrm{n}}+p_{\mathrm{n}} e^{\left(p_{n}+q_{n}\right) t_{f}}\right)}{p_{\mathrm{n}}+q_{\mathrm{n}}}-q_{\mathrm{n}}$. Let $a(t)=m_{3}^{\mathrm{n}}(t)-m_{2}^{\mathrm{n}}(t)$. Then, subtracting (14) from (15), we get

$$
\frac{d a}{d t}=a\left(-2 p_{\mathrm{nc}}+q_{\mathrm{n}}-\frac{q_{\mathrm{n}}}{\mathcal{M}} n(t)\right)
$$

Solving (A13) with the initial condition $a\left(t_{f}\right)=m_{3}^{\mathrm{n}}\left(t_{f}\right)$, we get $a(t)=\frac{m_{3}^{\mathrm{n}}\left(t_{f}\right) e^{\left(2\left(p_{\mathrm{n}}-p_{\mathrm{nc}}\right)+q_{\mathrm{n}}\right)\left(t-t_{f}\right)}\left(\alpha+q_{\mathrm{n}}\right)}{\alpha e^{\left(2 p_{\mathrm{n}}+q_{\mathrm{n}}\right)\left(t-t_{f}\right)}+q_{\mathrm{n}}}$. Combining this with $m_{2}^{\mathrm{n}}(t)+m_{3}^{\mathrm{n}}(t)=n(t)$ and, $m_{3}^{\mathrm{n}}(t)-m_{2}^{\mathrm{n}}(t)=a(t)$, we get the expressions for $m_{2}^{\mathrm{n}}(t)$ and $m_{3}^{\mathrm{n}}(t)$. Next, denote $m^{\mathrm{tot}}(t)=m_{1}^{\mathrm{o}}(t)+m_{2}^{\mathrm{o}}(t)+m_{2}^{\mathrm{n}}(t)+m_{3}^{\mathrm{n}}(t)$. Then, adding equations (12)-(15), we get

$$
\frac{d m^{\text {tot }}}{d t}=\left(2 p_{\mathrm{o}}+2 p_{\mathrm{n}}+q_{\mathrm{o}} \frac{m^{\mathrm{tot}}}{\mathcal{M}}+\left(\frac{q_{\mathrm{n}}-q_{\mathrm{o}}}{\mathcal{M}}\right) m_{3}^{\mathrm{n}}\right)\left(\mathcal{M}-m^{\mathrm{tot}}\right)
$$

Denoting $X(t)=\frac{\mathcal{M}}{\mathcal{M}-m^{\text {tot }}}$, we can rewrite $(\mathrm{A} 14)$ as

$$
\frac{d X}{d t}=X f(t)-q_{\mathrm{o}}
$$

with $f(t)=\left(2 p_{\mathrm{o}}+2 p_{\mathrm{n}}+q_{\mathrm{o}}+\frac{\left(q_{\mathrm{n}}-q_{\mathrm{o}}\right)}{\mathcal{M}} n(t)\right)$ and the initial condition $X\left(t_{f}\right)=\frac{\mathcal{M}}{\mathcal{M}-\mathcal{M} \widehat{G}_{I}\left(p_{\mathrm{o}}, p_{\mathrm{n}}, q_{\mathrm{o}}, q_{\mathrm{n}}, t_{f}\right)}$. The solution to (A15) with this initial condition is given by

$$
X(t)=\left(\frac{\mathcal{M}}{\mathcal{M}-\mathcal{M} \widehat{G}_{I}\left(p_{\mathrm{o}}, p_{\mathrm{n}}, q_{\mathrm{o}}, q_{\mathrm{n}}, t_{f}\right)}-q_{\mathrm{o}} \int_{t_{f}}^{t} d \tau e^{-\int_{t_{f}}^{\tau} f(u) d u}\right) \int_{t^{t_{f}}}^{t} f(\tau) d \tau
$$

Note that

$$
\begin{aligned}
\int_{t_{f}}^{t} f(\tau) d \tau & =\int_{t_{f}}^{t}\left(2 p_{\mathrm{o}}+2 p_{\mathrm{n}}+q_{\mathrm{o}}+\left(\frac{q_{\mathrm{n}}-q_{\mathrm{o}}}{\mathcal{M}}\right) n(\tau)\right) d \tau \\
& =\left(2 p_{\mathrm{o}}+2 p_{\mathrm{n}}+q_{\mathrm{n}}\right)\left(t-t_{f}\right)+\left(\frac{q_{\mathrm{n}}-q_{\mathrm{o}}}{q_{\mathrm{n}}}\right) \log \left(\frac{q_{\mathrm{n}} e^{-\left(q_{\mathrm{n}}+2 p_{\mathrm{n}}\right)\left(\tau-t_{f}\right)}+\alpha}{q_{\mathrm{n}}+\alpha}\right),
\end{aligned}
$$


and

$$
\begin{gathered}
\int_{t_{f}}^{t} d \tau e^{-\int_{t_{f}}^{\tau} f(u) d u}=\int_{t_{f}}^{t} d \tau e^{-\left(2 p_{\mathrm{o}}+2 p_{\mathrm{n}}+q_{\mathrm{n}}\right)\left(\tau-t_{f}\right)}\left(\frac{q_{\mathrm{n}}+\alpha}{q_{\mathrm{n}} e^{-\left(q_{\mathrm{n}}+2 p_{\mathrm{n}}\right)\left(\tau-t_{f}\right)}+\alpha}\right)^{\left(\frac{q_{\mathrm{n}}-q_{\mathrm{o}}}{q_{\mathrm{n}}}\right)} \\
=\frac{\alpha+q_{\mathrm{n}}}{\alpha\left(2 p_{\mathrm{o}}+2 p_{\mathrm{n}}+q_{\mathrm{n}}\right)}\left(\begin{array}{c}
{ }_{2} F_{1}\left(1, \frac{2 p_{\mathrm{o}}+2 p_{\mathrm{n}}+q_{\mathrm{n}}}{2 p_{\mathrm{n}}+q_{\mathrm{n}}}+\frac{q_{\mathrm{o}}}{q_{\mathrm{n}}} ; \frac{2\left(p_{\mathrm{o}}+2 p_{\mathrm{n}}+q_{\mathrm{n}}\right)}{2 p_{\mathrm{n}}+q_{\mathrm{n}}} ;-\frac{q_{\mathrm{n}}}{\alpha}\right. \\
-e^{-\left(2 p_{\mathrm{o}}+2 p_{\mathrm{n}}+q_{\mathrm{n}}\right)\left(t-t_{f}\right)}\left(\frac{\alpha+q_{\mathrm{n}} e^{-\left(2 p_{\mathrm{n}}+q_{\mathrm{n}}\right)\left(t-t_{f}\right)}}{\alpha+q_{\mathrm{n}}}\right)^{\frac{q_{\mathrm{o}}}{q_{\mathrm{n}}}} \\
\times{ }_{2} F_{1}\left(1, \frac{2 p_{\mathrm{o}}+2 p_{\mathrm{n}}+q_{\mathrm{n}}}{2 p_{\mathrm{n}}+q_{\mathrm{n}}}+\frac{q_{\mathrm{o}}}{q_{\mathrm{n}}} ; \frac{2\left(p_{\mathrm{o}}+2 p_{\mathrm{n}}+q_{\mathrm{n}}\right)}{2 p_{\mathrm{n}}+q_{\mathrm{n}}} ;-\frac{q_{\mathrm{n}}}{\alpha} e^{-\left(2 p_{\mathrm{n}}+q_{\mathrm{n}}\right)\left(t-t_{f}\right)}\right)
\end{array}\right),
\end{gathered}
$$

where ${ }_{2} F_{1}(a, b ; c ; x)$ is the hypergeometric function. Using $(20)$, we get

$$
\begin{aligned}
X(t)= & \left(\frac{1}{1-\widehat{G}_{I}\left(p_{\mathrm{o}}, p_{\mathrm{n}}, q_{\mathrm{o}}, q_{\mathrm{n}}, t_{f}\right)}-\frac{q_{\mathrm{o}}\left(\alpha+q_{\mathrm{n}}\right)}{\alpha\left(2 p_{\mathrm{o}}+2 p_{\mathrm{n}}+q_{\mathrm{n}}\right)} \widehat{F}_{I I}\left(p_{\mathrm{o}}, p_{\mathrm{n}}, q_{\mathrm{o}}, q_{\mathrm{n}}, t\right)\right) \\
& \times\left(\frac{\alpha+q_{\mathrm{n}} e^{-\left(q_{\mathrm{n}}+2 p_{\mathrm{n}}\right)\left(t-t_{f}\right)}}{\alpha+q_{\mathrm{n}}}\right)^{\left(\frac{q_{\mathrm{n}}-q_{\mathrm{o}}}{q_{\mathrm{n}}}\right)} \exp \left(\left(2 p_{\mathrm{o}}+2 p_{\mathrm{n}}+q_{\mathrm{n}}\right)\left(t-t_{f}\right)\right),
\end{aligned}
$$

and

$$
m^{\text {tot }}(t)=\mathcal{M}\left(1-\frac{e^{-\left(2 p_{\mathrm{o}}+2 p_{\mathrm{n}}+q_{\mathrm{n}}\right)\left(t-t_{f}\right)}\left(\frac{\left(q_{\mathrm{n}}+\alpha\right) e^{\left(q_{\mathrm{n}}+2 p_{\mathrm{n}}\right)\left(t-t_{f}\right)}}{q_{\mathrm{n}}+\alpha e^{\left(q_{\mathrm{n}}+2 p_{\mathrm{n}}\right)\left(t-t_{f}\right)}}\right)^{\left(\frac{q_{\mathrm{n}}-q_{\mathrm{o}}}{q_{\mathrm{n}}}\right)}}{\left(\frac{1}{1-\widehat{G}_{I}\left(p_{\mathrm{o}}, p_{\mathrm{n}}, q_{\mathrm{o}}, q_{\mathrm{n}}, t_{f}\right)}-\frac{q_{\mathrm{o}}\left(\alpha+q_{\mathrm{n}}\right)}{\alpha\left(2 p_{\mathrm{o}}+2 p_{\mathrm{n}}+q_{\mathrm{n}}\right)} \widehat{F}_{I I}\left(p_{\mathrm{o}}, p_{\mathrm{n}}, q_{\mathrm{o}}, q_{\mathrm{n}}, t\right)\right)}\right) .
$$

Now, using (21), defining $m^{-}(t)=m_{1}^{\circ}(t)-m_{2}^{\circ}(t)$ and subtracting (13) from (12), we get

$$
\frac{d m^{-}(t)}{d t}=\left(-2 p_{\mathrm{oc}}-2 p_{\mathrm{n}}+q_{\mathrm{o}}-q_{\mathrm{o}} \widehat{G}_{I I}\left(p_{\mathrm{o}}, p_{\mathrm{n}}, q_{\mathrm{o}}, q_{\mathrm{n}}, t\right)-\frac{q_{\mathrm{n}}}{\mathcal{M}} n(t)\right) m^{-}(t)
$$

with the initial condition $m^{-}\left(t_{f}\right)=\widehat{H}_{I}\left(p_{\mathrm{o}}, p_{\mathrm{oc}}, p_{\mathrm{n}}, q_{\mathrm{o}}, q_{\mathrm{n}}, t_{f}\right)$. The solution to (A21) with this initial condition is given by

$$
\begin{aligned}
m^{-}(t) & =\frac{\widehat{H}_{I}\left(p_{\mathrm{o}}, p_{\mathrm{oc}}, p_{\mathrm{n}}, q_{\mathrm{o}}, q_{\mathrm{n}}, t_{f}\right)\left(\alpha+q_{\mathrm{n}}\right) e^{\left(q_{\mathrm{o}}-2 p_{\mathrm{oc}}\right)\left(t-t_{f}\right)}}{q_{\mathrm{n}}+\alpha e^{\left(q_{\mathrm{n}}+2 p_{\mathrm{n}}\right)\left(t-t_{f}\right)}} e^{-q_{\mathrm{o}} \int_{t_{f}}^{t} \widehat{G}_{I I}\left(p_{\mathrm{o}}, p_{\mathrm{n}}, q_{\mathrm{o}}, q_{\mathrm{n}}, \tau\right) d \tau} \\
& =\widehat{H}_{I I}\left(p_{\mathrm{o}}, p_{\mathrm{oc}}, p_{\mathrm{n}}, q_{\mathrm{o}}, q_{\mathrm{n}}, t\right) .
\end{aligned}
$$

Combining the above with $m_{1}^{\circ}(t)+m_{2}^{\circ}(t)=m^{\text {tot }}(t)-n(t)$, we get the expressions for $m_{1}^{\circ}(t)$ and $m_{2}^{\circ}(t)$

\section{Proof of Corollary 2.1}


The customer base of the new entrant, $m_{3}^{\mathrm{n}}(t)$, and that of the second incumbent's new business model, $m_{2}^{\mathrm{n}}(t)$, remain identical to those in Proposition 2. Plugging in $q_{\mathrm{o}}=0$ into equation (A14) we get

$$
\frac{d m^{\text {tot }}}{d t}=\left(2 p_{\mathrm{o}}+2 p_{\mathrm{n}}+\frac{q_{\mathrm{n}}}{\mathcal{M}} n(t)\right)\left(\mathcal{M}-m^{\mathrm{tot}}\right) .
$$

Given the initial condition $m^{\text {tot }}\left(t_{f}\right)=\mathcal{M}-\frac{(\mathcal{M}-m-M)\left(2 p_{\mathrm{n}}+q_{\mathrm{n}}\right) e^{-2 p_{\mathrm{o}} t_{f}}}{q_{\mathrm{n}}+\alpha}$, the solution to (A23) is

$$
m^{\text {tot }}(t)=\mathcal{M}-\frac{(\mathcal{M}-m-M)\left(2 p_{\mathrm{n}}+q_{\mathrm{n}}\right) e^{-2 p_{\mathrm{o}} t}}{q_{\mathrm{n}}+\alpha e^{\left(q_{\mathrm{n}}+2 p_{\mathrm{n}}\right)\left(t-t_{f}\right)}} .
$$

Using, $m^{-}(t)=m_{1}^{\mathrm{o}}(t)-m_{2}^{\mathrm{o}}(t)$ and plugging in $q_{\mathrm{o}}=0$ into equation (A21), we get

$$
\frac{d m^{-}(t)}{d t}=\left(-2 p_{\mathrm{oc}}-2 p_{\mathrm{n}}-\frac{q_{\mathrm{n}}}{\mathcal{M}} n(t)\right) m^{-}(t)
$$

with the initial condition $m^{-}\left(t_{f}\right)=\frac{(M-m)\left(q_{\mathrm{n}}+p_{\mathrm{n}}\right) e^{-2 p_{\mathrm{oc}} t_{f}}}{q_{\mathrm{n}}+p_{\mathrm{n}} e^{\left(q_{\mathrm{n}}+p_{\mathrm{n}}\right) t_{f}}}$. The solution to (A25) with this initial condition is given by $m^{-}(t)=\frac{(M-m)\left(q_{\mathrm{n}}+2 p_{\mathrm{n}}\right) e^{-2 p_{\mathrm{oc}} t}}{q_{\mathrm{n}}+\alpha e^{\left(q_{\mathrm{n}}+2 p_{\mathrm{n}}\right)\left(t-t_{f}\right)}}$. Given that $m_{1}^{\mathrm{o}}(t)+m_{2}^{\mathrm{o}}(t)=m^{\mathrm{tot}}(t)-n(t)$, we get the expressions for $m_{1}^{\circ}(t)$ and $m_{2}^{\circ}(t)$.

\section{Proof of Proposition 3}

Plugging in the conditions of Proposition 3 into the diffusion equations (12)-(15), and using (??) we get:

$$
\begin{aligned}
& \frac{d o_{1}}{d t}=-2 p_{\mathrm{n}} o_{1}-Q_{\mathrm{n}} n_{2} o_{1}-q_{\mathrm{n}} n_{3} o_{1}, \\
& \frac{d o_{2}}{d t}=-2 p_{\mathrm{n}} o_{2}-Q_{\mathrm{n}} n_{2} o_{2}-q_{\mathrm{n}} n_{3} o_{2}, \\
& \frac{d n_{2}}{d t}=p_{\mathrm{n}}\left(o_{1}+o_{2}\right)+Q_{\mathrm{n}} n_{2}\left(o_{1}+o_{2}\right), \\
& \frac{d n_{3}}{d t}=p_{\mathrm{n}}\left(o_{1}+o_{2}\right)+q_{\mathrm{n}} n_{3}\left(o_{1}+o_{2}\right),
\end{aligned}
$$

with the initial conditions:

$$
\begin{aligned}
& o_{1}\left(t_{f}\right)=\theta\left(\frac{q_{\mathrm{n}}+p_{\mathrm{n}}}{q_{\mathrm{n}}+p_{\mathrm{n}} e^{\left(q_{\mathrm{n}}+p_{\mathrm{n}}\right) t_{f}}}\right), \\
& o_{2}\left(t_{f}\right)=(1-\theta)\left(\frac{q_{\mathrm{n}}+p_{\mathrm{n}}}{q_{\mathrm{n}}+p_{\mathrm{n}} e^{\left(q_{\mathrm{n}}+p_{\mathrm{n}}\right) t_{f}}}\right), \\
& n_{2}\left(t_{f}\right)=0, \\
& n_{3}\left(t_{f}\right)=1-\frac{q_{\mathrm{n}}+p_{\mathrm{n}}}{q_{\mathrm{n}}+p_{\mathrm{n}} e^{\left(q_{\mathrm{n}}+p_{\mathrm{n}}\right) t_{f}}} .
\end{aligned}
$$


Dividing (A26) by (A27), we get

$$
\frac{d o_{1}}{o_{1}}=\frac{d o_{2}}{o_{2}} .
$$

Using the initial conditions (A30) and (A31), we obtain, as in phase I,

$$
o_{2}(t)=o_{1}(t)\left(\frac{1-\theta}{\theta}\right) .
$$

Similarly, dividing (A28) by (A29), we have

$$
\frac{d n_{2}}{p_{\mathrm{n}}+Q_{\mathrm{n}} n_{2}}=\frac{d n_{3}}{p_{\mathrm{n}}+q_{\mathrm{n}} n_{3}} .
$$

For $q_{\mathrm{n}}>0$, integrating (A36) with initial conditions (A32) and (A33) yields

$$
n_{2}(t)=\frac{p_{\mathrm{n}}}{Q_{\mathrm{n}}}\left(\left(\frac{p_{\mathrm{n}}+q_{\mathrm{n}} n_{3}(t)}{p_{\mathrm{n}}+q_{\mathrm{n}} n_{3}\left(t_{f}\right)}\right)^{\frac{Q_{\mathrm{n}}}{q_{\mathrm{n}}}}-1\right) .
$$

Similarly, when $q_{\mathrm{n}}=0$, integration of (A36) with initial conditions (A32) and (A33) yields

$$
n_{2}(t)=\frac{p_{\mathrm{n}}}{Q_{\mathrm{n}}}\left(e^{\frac{Q_{\mathrm{n}}}{p_{\mathrm{n}}}\left(n_{3}(t)-n_{3}\left(t_{f}\right)\right)}-1\right) .
$$

Combining (A37) and (A29) with the conservation of market potential equation $o_{1}(t)+o_{2}(t)+$ $n_{2}(t)+n_{3}(t)=1$, we obtain

$$
\frac{d n_{3}}{d t}=\left(p_{\mathrm{n}}+q_{\mathrm{n}} n_{3}\right)\left(1-\frac{p_{\mathrm{n}}}{Q_{\mathrm{n}}}\left(\left(\frac{p_{\mathrm{n}}+q_{\mathrm{n}} n_{3}(t)}{p_{\mathrm{n}}+q_{\mathrm{n}} n_{3}\left(t_{f}\right)}\right)^{\frac{Q_{\mathrm{n}}}{q_{\mathrm{n}}}}-1\right)-n_{3}\right) .
$$

Using the definition (28), we get

$$
t-t_{f}=\int_{n_{3}\left(t_{f}\right)}^{n_{3}(t)} \frac{d y}{\left(p_{\mathrm{n}}+q_{\mathrm{n}} y\right)\left(1-S_{1}(y)-y\right)} .
$$

Combining (A40) with (A35) and $o_{1}(t)+o_{2}(t)+n_{2}(t)+n_{3}(t)=1$, we get $(29)$.

\section{Proof of Corollary 3.1}

For $Q_{\mathrm{n}}=q_{\mathrm{n}},(28)$ becomes

$$
S_{1}(x)=\frac{p_{\mathrm{n}}}{q_{\mathrm{n}}}\left(\left(\frac{p_{\mathrm{n}}+q_{\mathrm{n}} x}{p_{\mathrm{n}}+q_{\mathrm{n}} n_{3}\left(t_{f}\right)}\right)-1\right) .
$$


Then, (A40) can be expressed as

$$
t-t_{f}=\int_{n_{3}\left(t_{f}\right)}^{n_{3}(t)} \frac{\left(p_{\mathrm{n}}+q_{\mathrm{n}} n_{3}\left(t_{f}\right)\right) d y}{\left(p_{\mathrm{n}}+q_{\mathrm{n}} y\right)\left(p_{\mathrm{n}}\left(1-\frac{p_{\mathrm{n}}}{q_{\mathrm{n}}}\right)+\left(p_{\mathrm{n}}+q_{\mathrm{n}}\right) n_{3}\left(t_{f}\right)-\left(2 p_{\mathrm{n}}+q_{\mathrm{n}} n_{3}\left(t_{f}\right)\right) y\right)},
$$

or

$$
n_{3}(t)=A-\frac{\frac{p_{\mathrm{n}}}{q_{\mathrm{n}}}+A}{\left(\frac{\frac{p_{\mathrm{n}}}{q_{\mathrm{n}}}+n_{3}\left(t_{f}\right)}{A-n_{3}\left(t_{f}\right)}\right) \exp \left(\frac{q_{\mathrm{n}}\left(2 p_{\mathrm{n}}+q_{\mathrm{n}} n_{3}\left(t_{f}\right)\right)\left(\frac{p_{\mathrm{n}}}{q_{\mathrm{n}}}+A\right)\left(t-t_{f}\right)}{\left(p_{\mathrm{n}}+q_{\mathrm{n}} n_{3}\left(t_{f}\right)\right)}\right)+1} .
$$

where

$$
A=\frac{p_{\mathrm{n}}\left(1-\frac{p_{\mathrm{n}}}{q_{\mathrm{n}}}\right)+\left(p_{\mathrm{n}}+q_{\mathrm{n}}\right) n_{3}\left(t_{f}\right)}{2 p_{\mathrm{n}}+q_{\mathrm{n}} n_{3}\left(t_{f}\right)} .
$$

Using $n_{2}(t)=S_{1}\left(n_{3}(t)\right)$, we get

$$
n_{2}(t)=\frac{p_{\mathrm{n}}}{q_{\mathrm{n}}}\left(\left(\frac{p_{\mathrm{n}}+q_{\mathrm{n}} n_{3}(t)}{p_{\mathrm{n}}+q_{\mathrm{n}} n_{3}\left(t_{f}\right)}\right)-1\right) .
$$

Now, since $o_{1}(t)+o_{2}(t)+n_{2}(t)+n_{3}(t)=1$, and $o_{2}(t)=o_{1}(t)\left(\frac{1-\theta}{\theta}\right)$, we get

$$
\frac{o_{1}(t)}{\theta}+\frac{p_{\mathrm{n}}}{q_{\mathrm{n}}}\left(\left(\frac{p_{\mathrm{n}}+q_{\mathrm{n}} n_{3}(t)}{p_{\mathrm{n}}+q_{\mathrm{n}} n_{3}\left(t_{f}\right)}\right)-1\right)+n_{3}(t)=1,
$$

or, equivalently, $o_{1}(t)=\theta\left(1-n_{3}(t)-\frac{p_{\mathrm{n}}}{q_{\mathrm{n}}}\left(\left(\frac{p_{\mathrm{n}}+q_{\mathrm{n}} n_{3}(t)}{p_{\mathrm{n}}+q_{\mathrm{n}} n_{3}\left(t_{f}\right)}\right)-1\right)\right), o_{2}(t)=\frac{(1-\theta)}{\theta} o_{1}(t)$.

\section{Proof of Proposition 4}

Throughout the proof, we use $Q_{\mathrm{n}}=q_{\mathrm{n}}$. Denoting $n(t)=m_{1}^{\mathrm{n}}(t)+m_{2}^{\mathrm{n}}(t)+m_{3}^{\mathrm{n}}(t)$ and adding (33)(35), we get

$$
\frac{d n}{d t}=\left(3 p_{\mathrm{n}}+\frac{q_{\mathrm{n}}}{\mathcal{M}} n\right)(\mathcal{M}-n) .
$$

Similar to the analysis in previous phases, we get $n(t)=\mathcal{M}\left(1-\frac{3 p_{\mathrm{n}}+q_{\mathrm{n}}}{\lambda e^{\left(3 p_{\mathrm{n}}+q_{\mathrm{n}}\right)\left(t-t_{s}\right)}+q_{\mathrm{n}}}\right)$, where $\lambda=$ $\frac{\left(3 p_{\mathrm{n}}+q_{\mathrm{n}}\right)\left(q_{\mathrm{n}}+\alpha e^{\left(2 p_{n}+q_{n}\right)\left(t_{s}-t_{f}\right)}\right)}{2 p_{\mathrm{n}}+q_{\mathrm{n}}}-q_{\mathrm{n}}$. Denoting $\widehat{a}(t)=m_{3}^{\mathrm{n}}(t)-m_{2}^{\mathrm{n}}(t)$, and subtracting (35) from (34), we get

$$
\frac{d \widehat{a}}{d t}=\widehat{a}\left(-3 p_{\mathrm{nc}}+q_{\mathrm{n}}-\frac{q_{\mathrm{n}}}{\mathcal{M}} n(t)\right) .
$$

Solving (A48) with the initial condition $\widehat{a}\left(t_{s}\right)=m_{3}^{\mathrm{n}}\left(t_{s}\right)-m_{2}^{\mathrm{n}}\left(t_{s}\right)$, we get $\widehat{a}(t)=$ $\frac{\left(m_{3}^{\mathrm{n}}\left(t_{s}\right)-m_{2}^{\mathrm{n}}\left(t_{s}\right)\right) e^{\left(3\left(p_{\mathrm{n}}-p_{\mathrm{nc}}\right)+q_{\mathrm{n}}\right)\left(t-t_{s}\right)}\left(\lambda+q_{\mathrm{n}}\right)}{\lambda e^{\left(3 p_{\mathrm{n}}+q_{\mathrm{n}}\right)\left(t-t_{s}\right)}+q_{\mathrm{n}}}$. Similarly, let $\widehat{b}(t)=m_{2}^{\mathrm{n}}(t)-m_{1}^{\mathrm{n}}(t)$. Subtracting equation (33) from (34) for $Q_{\mathrm{n}}=q_{\mathrm{n}}$, we get

$$
\frac{\widehat{d b}}{d t}=\widehat{b}\left(-3 p_{\mathrm{nc}}+q_{\mathrm{n}}-\frac{q_{\mathrm{n}}}{\mathcal{M}} n(t)\right)
$$


Solving (A49) with the initial condition $\widehat{b}\left(t_{s}\right)=m_{2}^{\mathrm{n}}\left(t_{s}\right)$, we get $\widehat{b}(t)=\frac{m_{2}^{\mathrm{n}}\left(t_{s}\right) e^{\left(3\left(p_{\mathrm{n}}-p_{\mathrm{nc}}\right)+q_{\mathrm{n}}\right)\left(t-t_{s}\right)}\left(\lambda+q_{\mathrm{n}}\right)}{\lambda e^{\left(3 p_{\mathrm{n}}+q_{\mathrm{n}}\right)\left(t-t_{s}\right)}+q_{\mathrm{n}}}$.

Combining the above with $m_{1}^{\mathrm{n}}(t)+m_{2}^{\mathrm{n}}(t)+m_{3}^{\mathrm{n}}(t)=n(t), m_{3}^{\mathrm{n}}(t)-m_{2}^{\mathrm{n}}(t)=\widehat{a}(t)$, and, $m_{2}^{\mathrm{n}}(t)-m_{1}^{\mathrm{n}}(t)=$ $\widehat{b}(t)$ we get the expressions for $m_{1}^{\mathrm{n}}(t), m_{2}^{\mathrm{n}}(t)$ and $m_{3}^{\mathrm{n}}(t)$. Further, let $m^{\text {tot }}(t)=m_{1}^{\mathrm{o}}(t)+m_{2}^{\mathrm{o}}(t)+$ $m_{1}^{\mathrm{n}}(t)+m_{2}^{\mathrm{n}}(t)+m_{3}^{\mathrm{n}}(t)$. Then, adding equations (31)-(35), we get

$$
\frac{d m^{\mathrm{tot}}}{d t}=\left(2 p_{\mathrm{o}}+3 p_{\mathrm{n}}+q_{\mathrm{o}} \frac{m^{\mathrm{tot}}}{\mathcal{M}}+\left(\frac{q_{\mathrm{n}}-q_{\mathrm{o}}}{\mathcal{M}}\right) n(t)\right)\left(\mathcal{M}-m^{\mathrm{tot}}\right) .
$$

Denoting $X(t)=\frac{\mathcal{M}}{\mathcal{M}-m^{\text {tot }}}$, we can rewrite $($ A50) as

$$
\frac{d X}{d t}=X f(t)-q_{\mathrm{o}}
$$

with $f(t)=\left(2 p_{\mathrm{o}}+3 p_{\mathrm{n}}+q_{\mathrm{o}}+\frac{\left(q_{\mathrm{n}}-q_{\mathrm{o}}\right)}{\mathcal{M}} n(t)\right)$ and the initial condition $X\left(t_{f}\right)=\frac{\mathcal{M}}{\mathcal{M}-\mathcal{M} \widehat{G}_{I I}\left(p_{\mathrm{o}}, p_{\mathrm{n}}, q_{\mathrm{o}}, q_{\mathrm{n}}, t_{s}\right)}$. The solution to (A51) with this initial condition is given by

$$
X(t)=\left(\frac{\mathcal{M}}{\mathcal{M}-\mathcal{M} \widehat{G}_{I I}\left(p_{\mathrm{o}}, p_{\mathrm{n}}, q_{\mathrm{o}}, q_{\mathrm{n}}, t_{s}\right)}-q_{\mathrm{o}} \int_{t_{s}}^{t} d \tau e^{-\int_{t_{s}}^{\tau} f(u) d u}\right) \int_{t^{t_{s}}}^{t} f(\tau) d \tau
$$

Note that

$$
\begin{aligned}
\int_{t_{f}}^{t} f(\tau) d \tau & =\int_{t_{f}}^{t}\left(2 p_{\mathrm{o}}+3 p_{\mathrm{n}}+q_{\mathrm{o}}+\left(\frac{q_{\mathrm{n}}-q_{\mathrm{o}}}{\mathcal{M}}\right) n(\tau)\right) d \tau \\
& =\left(2 p_{\mathrm{o}}+3 p_{\mathrm{n}}+q_{\mathrm{n}}\right)\left(t-t_{s}\right)+\left(\frac{q_{\mathrm{n}}-q_{\mathrm{o}}}{q_{\mathrm{n}}}\right) \log \left(\frac{q_{\mathrm{n}} e^{-\left(q_{\mathrm{n}}+3 p_{\mathrm{n}}\right)\left(\tau-t_{s}\right)}+\lambda}{q_{\mathrm{n}}+\lambda}\right)
\end{aligned}
$$

and

$$
\begin{aligned}
& \int_{t_{s}}^{t} d \tau e^{-\int_{t_{s}}^{\tau} f(u) d u} \\
= & \int_{t_{f}}^{t} d \tau e^{-\left(2 p_{\mathrm{o}}+3 p_{\mathrm{n}}+q_{\mathrm{n}}\right)\left(\tau-t_{s}\right)}\left(\frac{q_{\mathrm{n}}+\lambda}{q_{\mathrm{n}} e^{-\left(q_{\mathrm{n}}+3 p_{\mathrm{n}}\right)\left(\tau-t_{s}\right)}+\lambda}\right)^{\left(\frac{q_{\mathrm{n}}-q_{\mathrm{o}}}{q_{\mathrm{n}}}\right)} \\
= & \frac{{ }_{2} F_{1}\left(1, \frac{2 p_{\mathrm{o}}+3 p_{\mathrm{n}}+q_{\mathrm{n}}}{3 p_{\mathrm{n}}+q_{\mathrm{n}}}+\frac{q_{\mathrm{o}}}{q_{\mathrm{n}}} ; \frac{2\left(p_{\mathrm{o}}+3 p_{\mathrm{n}}+q_{\mathrm{n}}\right)}{3 p_{\mathrm{n}}+q_{\mathrm{n}}} ;-\frac{q_{\mathrm{n}}}{\lambda}\right)}{\lambda\left(2 p_{\mathrm{o}}+3 p_{\mathrm{n}}+q_{\mathrm{n}}\right)}\left(\begin{array}{c}
-q_{\mathrm{n}} \\
-e^{-\left(2 p_{\mathrm{o}}+3 p_{\mathrm{n}}+q_{\mathrm{n}}\right)\left(t-t_{s}\right)}\left(\frac{\lambda+q_{\mathrm{n}} e^{-\left(3 p_{\mathrm{n}}+q_{\mathrm{n}}\right)\left(t-t_{s}\right)}}{\lambda+q_{\mathrm{n}}}\right)^{\frac{q_{\mathrm{o}}}{q_{\mathrm{n}}}} \\
\times{ }_{2} F_{1}\left(1, \frac{2 p_{\mathrm{o}}+3 p_{\mathrm{n}}+q_{\mathrm{n}}}{3 p_{\mathrm{n}}+q_{\mathrm{n}}}+\frac{q_{\mathrm{o}}}{q_{\mathrm{n}}} ; \frac{2\left(p_{\mathrm{o}}+3 p_{\mathrm{n}}+q_{\mathrm{n}}\right)}{3 p_{\mathrm{n}}+q_{\mathrm{n}}} ;-\frac{q_{\mathrm{n}}}{\lambda} e^{-\left(3 p_{\mathrm{n}}+q_{\mathrm{n}}\right)\left(t-t_{s}\right)}\right)
\end{array}\right),
\end{aligned}
$$


where ${ }_{2} F_{1}(a, b ; c ; x)$ is the hypergeometric function. Using (40), we get

$$
\begin{aligned}
X(t)= & \left(\frac{1}{1-\widehat{G}_{I I}\left(p_{\mathrm{o}}, p_{\mathrm{n}}, q_{\mathrm{o}}, q_{\mathrm{n}}, t_{s}\right)}-\frac{q_{\mathrm{o}}\left(\lambda+q_{\mathrm{n}}\right)}{\lambda\left(2 p_{\mathrm{o}}+3 p_{\mathrm{n}}+q_{\mathrm{n}}\right)} \widehat{F}_{I I I}\left(p_{\mathrm{o}}, p_{\mathrm{n}}, q_{\mathrm{o}}, q_{\mathrm{n}}, t\right)\right) \\
& \times\left(\frac{\lambda+q_{\mathrm{n}} e^{-\left(q_{\mathrm{n}}+3 p_{\mathrm{n}}\right)\left(t-t_{s}\right)}}{\lambda+q_{\mathrm{n}}}\right)^{\left(\frac{q_{\mathrm{n}}-q_{\mathrm{o}}}{q_{\mathrm{n}}}\right)} \exp \left(\left(2 p_{\mathrm{o}}+3 p_{\mathrm{n}}+q_{\mathrm{n}}\right)\left(t-t_{s}\right)\right),
\end{aligned}
$$

and

$$
m^{\mathrm{tot}}(t)=\mathcal{M}\left(1-\frac{e^{-\left(2 p_{\mathrm{o}}+3 p_{\mathrm{n}}+q_{\mathrm{n}}\right)\left(t-t_{s}\right)}\left(\frac{\left(q_{\mathrm{n}}+\lambda\right) e^{\left(q_{\mathrm{n}}+3 p_{\mathrm{n}}\right)\left(t-t_{s}\right)}}{q_{\mathrm{n}}+\lambda e^{\left(q_{\mathrm{n}}+3 p_{\mathrm{n}}\right)\left(t-t_{s}\right)}}\right)^{\left(\frac{q_{\mathrm{n}}-q_{\mathrm{o}}}{q_{\mathrm{n}}}\right)}}{\left(\frac{1}{1-\widehat{G}_{I I}\left(p_{\mathrm{o}}, p_{\mathrm{n}}, q_{\mathrm{o}}, q_{\mathrm{n}}, t_{s}\right)}-\frac{q_{\mathrm{o}}\left(\lambda+q_{\mathrm{n}}\right)}{\lambda\left(2 p_{\mathrm{o}}+3 p_{\mathrm{n}}+q_{\mathrm{n}}\right)} \widehat{F}_{I I I}\left(p_{\mathrm{o}}, p_{\mathrm{n}}, q_{\mathrm{o}}, q_{\mathrm{n}}, t\right)\right)}\right) .
$$

Now, using (41), defining $m^{-}(t)=m_{1}^{\circ}(t)-m_{2}^{\circ}(t)$ and subtracting (32) from (31), we get

$$
\frac{d m^{-}(t)}{d t}=\left(-2 p_{\mathrm{oc}}-3 p_{\mathrm{n}}+q_{\mathrm{o}}-q_{\mathrm{o}} \widehat{G}_{I I I}\left(p_{\mathrm{o}}, p_{\mathrm{n}}, q_{\mathrm{o}}, q_{\mathrm{n}}, t\right)-\frac{q_{\mathrm{n}}}{\mathcal{M}} n(t)\right) m^{-}(t),
$$

with the initial condition $m^{-}\left(t_{s}\right)=\widehat{H}_{I I}\left(p_{\mathrm{o}}, p_{\mathrm{oc}}, p_{\mathrm{n}}, q_{\mathrm{o}}, q_{\mathrm{n}}, t_{s}\right)$. The solution to (A57) with this initial condition is given by $m^{-}(t)=\widehat{H}_{I I I}\left(p_{\mathrm{o}}, p_{\mathrm{oc}}, p_{\mathrm{n}}, q_{\mathrm{o}}, q_{\mathrm{n}}, t\right)$. Combining the above with $m_{1}^{\mathrm{o}}(t)+m_{2}^{\mathrm{o}}(t)=$ $m^{\text {tot }}(t)-n(t)$, we get the expressions for $m_{1}^{\circ}(t)$ and $m_{2}^{\circ}(t)$.

\section{Proof of Corollary 4.1}

The customer base of the new entrant, $m_{3}^{\mathrm{n}}(t)$, and those for the incumbents' new business models, $m_{1}^{\mathrm{n}}(t), m_{2}^{\mathrm{n}}(t)$, remain identical to those in Proposition 4. Plugging in $q_{\mathrm{o}}=0$ into (A50) we get

$$
\frac{d m^{\text {tot }}}{d t}=\left(2 p_{\mathrm{o}}+3 p_{\mathrm{n}}+\frac{q_{\mathrm{n}}}{\mathcal{M}} n(t)\right)\left(\mathcal{M}-m^{\mathrm{tot}}\right)
$$

Given the initial condition $m^{\text {tot }}\left(t_{s}\right)=\mathcal{M}-\frac{(\mathcal{M}-m-M)\left(3 p_{\mathrm{n}}+q_{\mathrm{n}}\right) e^{-2 p_{\mathrm{o}} t_{s}}}{q_{\mathrm{n}}+\lambda}$, the solution to (A58) becomes

$$
m^{\text {tot }}(t)=\mathcal{M}-\frac{(\mathcal{M}-m-M)\left(3 p_{\mathrm{n}}+q_{\mathrm{n}}\right) e^{-2 p_{\mathrm{o}} t}}{q_{\mathrm{n}}+\lambda e^{\left(q_{\mathrm{n}}+3 p_{\mathrm{n}}\right)\left(t-t_{s}\right)}} .
$$

Using, $m^{-}(t)=m_{1}^{\circ}(t)-m_{2}^{\circ}(t)$ and plugging in $q_{\mathrm{o}}=0$ into equation (A57), we get

$$
\frac{d m^{-}(t)}{d t}=\left(-2 p_{\mathrm{oc}}-3 p_{\mathrm{n}}-\frac{q_{\mathrm{n}}}{\mathcal{M}} n(t)\right) m^{-}(t)
$$

with the initial condition $m^{-}\left(t_{s}\right)=\frac{(M-m)\left(q_{\mathrm{n}}+2 p_{\mathrm{n}}\right) e^{-2 p_{\mathrm{oc}} t_{s}}}{q_{\mathrm{n}}+\alpha e^{\left(q_{\mathrm{n}}+2 p_{\mathrm{n}}\right)\left(t_{s}-t_{f}\right)}}$. The solution to (A60) with this initial condition is given by

$$
m^{-}(t)=\frac{(M-m)\left(q_{\mathrm{n}}+3 p_{\mathrm{n}}\right) e^{-2 p_{\mathrm{oc}} t}}{q_{\mathrm{n}}+\lambda e^{\left(q_{\mathrm{n}}+3 p_{\mathrm{n}}\right)\left(t-t_{s}\right)}}
$$


Given that $m_{1}^{\circ}(t)+m_{2}^{\circ}(t)=m^{\text {tot }}(t)-n(t)$, we get the final expressions for $m_{1}^{\circ}(t)$ and $m_{2}^{\circ}(t)$.

\section{Proof of Proposition 5}

Plugging in the conditions of Proposition 5 into (31)-(35), and using (??) we have:

$$
\begin{aligned}
\frac{d o_{1}}{d t} & =-3 p_{\mathrm{n}} o_{1}-Q_{\mathrm{n}}\left(n_{1}+n_{2}\right) o_{1}-q_{\mathrm{n}} n_{3} o_{1}, \\
\frac{d o_{2}}{d t} & =-3 p_{\mathrm{n}} o_{2}-Q_{\mathrm{n}}\left(n_{1}+n_{2}\right) o_{2}-q_{\mathrm{n}} n_{3} o_{2}, \\
\frac{d n_{1}}{d t} & =p_{\mathrm{n}}\left(o_{1}+o_{2}\right)+Q_{\mathrm{n}} n_{1}\left(o_{1}+o_{2}\right), \\
\frac{d n_{2}}{d t} & =p_{\mathrm{n}}\left(o_{1}+o_{2}\right)+Q_{\mathrm{n}} n_{2}\left(o_{1}+o_{2}\right), \\
\frac{d n_{3}}{d t} & =p_{\mathrm{n}}\left(o_{1}+o_{2}\right)+q_{\mathrm{n}} n_{3}\left(o_{1}+o_{2}\right),
\end{aligned}
$$

with the initial conditions:

$$
\begin{aligned}
o_{1}\left(t_{s}\right) & =\theta\left(1-n_{3}\left(t_{s}\right)-S\left(n_{3}\left(t_{s}\right), n_{3}\left(t_{f}\right)\right)\right), \\
o_{2}\left(t_{s}\right) & =(1-\theta)\left(1-n_{3}\left(t_{s}\right)-S\left(n_{3}\left(t_{s}\right), n_{3}\left(t_{f}\right)\right)\right), \\
n_{1}\left(t_{s}\right) & =0, \\
n_{2}(t) & =S\left(n_{3}\left(t_{s}\right), n_{3}\left(t_{f}\right)\right), \\
t_{s}-t_{f} & =\int_{n_{3}\left(t_{f}\right)}^{n_{3}\left(t_{s}\right)} \frac{d y}{\left(p_{\mathrm{n}}+q_{\mathrm{n}} y\right)\left(1-y-S\left(y, n_{3}\left(t_{f}\right)\right)\right)} .
\end{aligned}
$$

Combining (A63) and (A64), we get

$$
\frac{d n_{1}}{p_{\mathrm{n}}+Q_{\mathrm{n}} n_{1}}=\frac{d n_{2}}{p_{\mathrm{n}}+Q_{\mathrm{n}} n_{2}},
$$

or

$$
n_{1}(t)=\frac{p_{\mathrm{n}}}{Q_{\mathrm{n}}}\left(\left(\frac{p_{\mathrm{n}}+Q_{\mathrm{n}} n_{2}(t)}{p_{\mathrm{n}}+Q_{\mathrm{n}} n_{2}\left(t_{s}\right)}\right)-1\right) .
$$

Similarly, putting together (A63) and (A70) yields

$$
n_{1}(t)=\frac{p_{\mathrm{n}}}{Q_{\mathrm{n}}}\left(\left(\frac{p_{\mathrm{n}}+q_{\mathrm{n}} n_{3}(t)}{p_{\mathrm{n}}+q_{\mathrm{n}} n\left(t_{s}\right)}\right)^{\frac{Q_{\mathrm{n}}}{q_{\mathrm{n}}}}-1\right) .
$$

Denoting

$$
S_{2}(x)=\frac{p_{\mathrm{n}}}{Q_{\mathrm{n}}}\left(\left(\frac{p_{\mathrm{n}}+q_{\mathrm{n}} x}{p_{\mathrm{n}}+q_{\mathrm{n}} n_{3}\left(t_{s}\right)}\right)^{\frac{Q_{\mathrm{n}}}{q_{\mathrm{n}}}}-1\right)
$$


and using $o_{1}(t)+o_{2}(t)+n_{1}(t)+n_{2}(t)+n_{3}(t)=1$, we convert (A70) into

$$
\frac{d n_{3}}{d t}=\left(p_{\mathrm{n}}+q_{\mathrm{n}} n_{3}\right)\left(1-n_{2}\left(t_{s}\right)-S_{2}\left(n_{3}\right)\left(2+\frac{Q_{\mathrm{n}}}{p_{\mathrm{n}}} n_{2}\left(t_{s}\right)\right)-n_{3}\right),
$$

so that

$$
t-t_{s}=\int_{n_{3}\left(t_{s}\right)}^{n_{3}(t)} \frac{d y}{\left(p_{\mathrm{n}}+q_{\mathrm{n}} y\right)\left(1-n_{2}\left(t_{s}\right)-y-S_{2}(y)\left(2+\frac{Q_{\mathrm{n}}}{p_{\mathrm{n}}} n_{2}\left(t_{s}\right)\right)\right)} .
$$

Further, combining (A61) and (A63), we get

$$
\frac{d o_{1}}{o_{1}}=\frac{d o_{2}}{o_{2}}
$$

and

$$
o_{2}(t)=\frac{o_{2}\left(t_{s}\right)}{o_{1}\left(t_{s}\right)} o_{1}(t)
$$

Finally, from $o_{1}(t)+o_{2}(t)+n_{1}(t)+n_{2}(t)+n_{3}(t)=1$ we obtain

$$
o_{1}(t)=\left(\frac{o_{1}\left(t_{s}\right)}{o_{1}\left(t_{s}\right)+o_{2}\left(t_{s}\right)}\right)\left(1-n_{3}(t)-S_{2}\left(n_{3}(t)\right)\left(2+\frac{Q_{\mathrm{n}} n_{2}\left(t_{s}\right)}{p_{\mathrm{n}}}\right)-n_{2}\left(t_{s}\right)\right)
$$

and

$$
o_{2}(t)=\left(\frac{o_{2}\left(t_{s}\right)}{o_{1}\left(t_{s}\right)+o_{2}\left(t_{s}\right)}\right)\left(1-n_{3}(t)-S_{2}\left(n_{3}(t)\right)\left(2+\frac{Q_{\mathrm{n}} n_{2}\left(t_{s}\right)}{p_{\mathrm{n}}}\right)-n_{2}\left(t_{s}\right)\right) .
$$

\section{Proof of Corollary 5.1}

For $q_{\mathrm{n}}=Q_{\mathrm{n}},(28)$ becomes $S_{2}(x)=p_{\mathrm{n}}\left(\frac{x-n_{3}\left(t_{s}\right)}{p_{\mathrm{n}}+q_{\mathrm{n}} n_{3}\left(t_{s}\right)}\right)$, and

$$
\begin{aligned}
t-t_{s} & =\int_{n_{3}\left(t_{s}\right)}^{n_{3}(t)} \frac{d y}{\left(p_{\mathrm{n}}+q_{\mathrm{n}} y\right)\left(1-n_{2}\left(t_{s}\right)-y-S_{2}(y)\left(2+\frac{q_{\mathrm{n}}}{p_{\mathrm{n}}} n_{2}\left(t_{s}\right)\right)\right)} \\
& =\int_{n_{3}\left(t_{s}\right)}^{n_{3}(t)} \frac{d y}{\left(\frac{p_{\mathrm{n}}}{q_{\mathrm{n}}}+y\right)\left(\frac{\left(1-n_{2}\left(t_{s}\right)\right) p_{\mathrm{n}}+n_{3}\left(t_{s}\right)\left(2 p_{\mathrm{n}}+q_{\mathrm{n}}\right)}{3 p_{\mathrm{n}}+q_{\mathrm{n}}\left(n_{3}\left(t_{s}\right)+n_{2}\left(t_{s}\right)\right)}-y\right)} \\
& =\left(\frac{1}{q_{\mathrm{n}}+3 p_{\mathrm{n}}}\right) \log \left(\frac{\left(1-n_{2}\left(t_{s}\right)-n_{3}\left(t_{s}\right)\right)\left(p_{\mathrm{n}}+q_{\mathrm{n}} n_{3}(t)\right)}{\left(1-n_{2}\left(t_{s}\right)-n_{3}\left(t_{s}\right)\right)\left(p_{\mathrm{n}}+q_{\mathrm{n}} n_{3}(t)\right)+\left(n_{3}\left(t_{s}\right)-n_{3}(t)\right)\left(q_{\mathrm{n}}+3 p_{\mathrm{n}}\right)}\right) .
\end{aligned}
$$

The last expression is equivalent to

$$
n_{3}(t)=\frac{n_{3}\left(t_{s}\right)\left(q_{\mathrm{n}}+3 p_{\mathrm{n}}\right)+p_{\mathrm{n}}\left(1-\exp \left(-\left(q_{\mathrm{n}}+3 p_{\mathrm{n}}\right)\left(t-t_{s}\right)\right)\right)\left(1-n_{2}\left(t_{s}\right)-n_{3}\left(t_{s}\right)\right)}{\left(q_{\mathrm{n}}+3 p_{\mathrm{n}}\right)-q_{\mathrm{n}}\left(1-\exp \left(-\left(q_{\mathrm{n}}+3 p_{\mathrm{n}}\right)\left(t-t_{s}\right)\right)\right)\left(1-n_{2}\left(t_{s}\right)-n_{3}\left(t_{s}\right)\right)} .
$$

Finally, substituting (A82) into (47), we get (48). 


\section{Proof of Proposition 6}

a) Using the expression for $n(t)=m_{1}^{\mathrm{n}}(t)+m_{2}^{\mathrm{n}}(t)+m_{3}^{\mathrm{n}}(t)$ from (43), and taking the limit we have:

$$
\lim _{t \rightarrow \infty} n(t)=\lim _{t \rightarrow \infty} \mathcal{M}\left(1-\frac{3 p_{\mathrm{n}}+q_{\mathrm{n}}}{\lambda e^{\left(3 p_{\mathrm{n}}+q_{\mathrm{n}}\right)\left(t-t_{s}\right)}+q_{\mathrm{n}}}\right)=\mathcal{M},
$$

Then, using $\widehat{a}(t)=m_{3}^{\mathrm{n}}(t)-m_{2}^{\mathrm{n}}(t)$ and taking the limit we get

$$
\begin{aligned}
\lim _{t \rightarrow \infty} \widehat{a}(t) & =\frac{a\left(t_{s}\right) e^{\left(3\left(p_{\mathrm{n}}-p_{\mathrm{nc}}\right)+q_{\mathrm{n}}\right)\left(t-t_{s}\right)}\left(\lambda+q_{\mathrm{n}}\right)}{\lambda e^{\left(3 p_{\mathrm{n}}+q_{\mathrm{n}}\right)\left(t-t_{s}\right)}+q_{\mathrm{n}}} \\
& =\lim _{t \rightarrow \infty} \frac{a\left(t_{s}\right)\left(\lambda+q_{\mathrm{n}}\right)}{\lambda e^{3 p_{\mathrm{nc}}\left(t-t_{s}\right)}+q_{\mathrm{n}} e^{-\left(3\left(p_{\mathrm{n}}-p_{\mathrm{nc}}\right)+q_{\mathrm{n}}\right)\left(t-t_{s}\right)}}=0 .
\end{aligned}
$$

For $\widehat{b}(t)=m_{2}^{\mathrm{n}}(t)-m_{1}^{\mathrm{n}}(t)$ the limit gets us

$$
\begin{aligned}
\lim _{t \rightarrow \infty} \widehat{b}(t) & =\lim _{t \rightarrow \infty} \frac{m_{2}^{\mathrm{n}}\left(t_{s}\right) e^{\left(3\left(p_{\mathrm{n}}-p_{\mathrm{nc}}\right)+q_{\mathrm{n}}\right)\left(t-t_{s}\right)}\left(\lambda+q_{\mathrm{n}}\right)}{\lambda e^{\left(3 p_{\mathrm{n}}+q_{\mathrm{n}}\right)\left(t-t_{s}\right)}+q_{\mathrm{n}}} \\
& =\lim _{t \rightarrow \infty} \frac{m_{2}^{\mathrm{n}}\left(t_{s}\right)\left(\lambda+q_{\mathrm{n}}\right)}{\lambda e^{3 p_{\mathrm{nc}}\left(t-t_{s}\right)}+q_{\mathrm{n}} e^{-\left(3\left(p_{\mathrm{n}}-p_{\mathrm{nc}}\right)+q_{\mathrm{n}}\right)\left(t-t_{s}\right)}}=0 .
\end{aligned}
$$

Combining equations (A83)-(A85) yields $m_{1}^{\infty}=m_{2}^{\infty}=m_{3}^{\infty}=\frac{\mathcal{M}}{3}$.

b) Note that in Corollary 3.2, $o_{1}(\infty)=o_{2}(\infty)=0$. Then, as it follows from (47),

$$
1-n_{3}^{\infty}-S\left(n_{3}^{\infty}, n_{3}\left(t_{s}\right)\right)\left(2+\frac{Q_{\mathrm{n}}}{p_{\mathrm{n}}} S\left(n_{3}\left(t_{s}\right), n_{3}\left(t_{f}\right)\right)\right)-S\left(n_{3}\left(t_{s}\right), n_{3}\left(t_{f}\right)\right)=0 .
$$

Introducing $\gamma=\frac{p_{\mathrm{n}}}{Q_{\mathrm{n}}}$ and $\delta=\frac{Q_{\mathrm{n}}}{q_{\mathrm{n}}}$, we can transform (A86) into

$$
n_{3}^{\infty}=1-\frac{\gamma}{\delta}\left(\left(\frac{\gamma+n_{3}^{\infty}}{\gamma+n_{3}\left(t_{s}\right)}\right)^{\delta}-1\right)\left(1+\left(\frac{\gamma+n_{3}\left(t_{s}\right)}{\gamma+n_{3}\left(t_{f}\right)}\right)^{\delta}\right)-\frac{\gamma}{\delta}\left(\left(\frac{\gamma+n\left(t_{s}\right)}{\gamma+n_{3}\left(t_{f}\right)}\right)^{\delta}-1\right)
$$

which, in turn, is equivalent to (51). Further, (52) are obtained by substituting (A87) into the last 2 equations in (47). For $Q_{\mathrm{n}}=q_{\mathrm{n}}(51)$ becomes

$$
1-\frac{\gamma\left(n_{3}\left(t_{s}\right)-n_{3}\left(t_{f}\right)\right)}{\gamma+n_{3}\left(t_{f}\right)}-n_{3}^{\infty}=\gamma\left(2+\frac{n_{3}\left(t_{s}\right)-n_{3}\left(t_{f}\right)}{\gamma+n_{3}\left(t_{f}\right)}\right)\left(\frac{n_{3}^{\infty}-n_{3}\left(t_{s}\right)}{\gamma+n_{3}\left(t_{s}\right)}\right),
$$

or, equivalently,

$$
\begin{aligned}
n_{3}^{\infty} & =\frac{\left(\gamma+n_{3}\left(t_{f}\right)-\gamma n_{3}\left(t_{s}\right)+\gamma n_{3}\left(t_{f}\right)\right)\left(\gamma+n_{3}\left(t_{s}\right)\right)+\gamma\left(2 \gamma+n_{3}\left(t_{s}\right)+n_{3}\left(t_{f}\right)\right) n_{3}\left(t_{s}\right)}{\left(\gamma+n_{3}\left(t_{f}\right)\right)\left(\gamma+n_{3}\left(t_{s}\right)\right)+\gamma\left(2 \gamma+n_{3}\left(t_{s}\right)+n_{3}\left(t_{f}\right)\right)} \\
& =\frac{\left.(3 \gamma+1)\left(\gamma+n_{3}\left(t_{f}\right)\right)\right)\left(\gamma+n_{3}\left(t_{s}\right)\right)}{\left(\gamma+n_{3}\left(t_{f}\right)\right)\left(\gamma+n_{3}\left(t_{s}\right)\right)+\gamma\left(2 \gamma+n_{3}\left(t_{s}\right)+n_{3}\left(t_{f}\right)\right)}-\gamma .
\end{aligned}
$$


Then, using (52), one gets

$$
n_{1}^{\infty}=\frac{\gamma}{\gamma+n_{3}\left(t_{s}\right)}\left(n_{3}^{\infty}-n_{3}\left(t_{s}\right)\right)=\frac{\left.\gamma(3 \gamma+1)\left(\gamma+n_{3}\left(t_{f}\right)\right)\right)}{\left(\gamma+n_{3}\left(t_{f}\right)\right)\left(\gamma+n_{3}\left(t_{s}\right)\right)+\gamma\left(2 \gamma+n_{3}\left(t_{s}\right)+n_{3}\left(t_{f}\right)\right)}-\gamma,
$$

and

$$
n_{2}^{\infty}=\frac{\left.\gamma(3 \gamma+1)\left(\gamma+n_{3}\left(t_{s}\right)\right)\right)}{\left(\gamma+n_{3}\left(t_{f}\right)\right)\left(\gamma+n_{3}\left(t_{s}\right)\right)+\gamma\left(2 \gamma+n_{3}\left(t_{s}\right)+n_{3}\left(t_{f}\right)\right)}-\gamma
$$

Differentiating (51) with respect to $n_{3}\left(t_{s}\right)$, we get

$$
\frac{\partial n_{3}^{\infty}}{\partial n_{3}\left(t_{s}\right)}=\frac{\gamma\left(\left(\frac{\gamma+n_{3}^{\infty}}{\gamma+n_{3}\left(t_{s}\right)}\right)^{\delta}\right)\left(\frac{1}{\gamma+n_{3}\left(t_{s}\right)}\right)}{1+\gamma\left(\left(\frac{\gamma+n_{3}^{\infty}}{\gamma+n_{3}\left(t_{f}\right)}\right)^{\delta-1}\left(\frac{1}{\gamma+n_{3}\left(t_{f}\right)}\right)+\left(\frac{\gamma+n_{3}^{\infty}}{\gamma+n_{3}\left(t_{s}\right)}\right)^{\delta-1}\left(\frac{1}{\gamma+n_{3}\left(t_{s}\right)}\right)\right)}>0 .
$$

Monotonicity of $n_{3}^{\infty}$ with respect to $n_{3}\left(t_{f}\right)$ is shown in exactly same way. Further, using (52), we get

$$
\frac{\partial n_{1}^{\infty}}{\partial n_{3}\left(t_{s}\right)}=\gamma\left(\left(\frac{\gamma+n_{3}^{\infty}}{\gamma+n_{3}\left(t_{s}\right)}\right)^{\delta-1}\left(\frac{1}{\gamma+n_{3}\left(t_{s}\right)}\right)\left(\frac{\partial n_{3}^{\infty}}{\partial n_{3}\left(t_{s}\right)}-\frac{\gamma+n_{3}^{\infty}}{\gamma+n_{3}\left(t_{s}\right)}\right)\right)
$$

Now,

$$
\begin{aligned}
& \frac{\partial n_{3}^{\infty}}{\partial n_{3}\left(t_{s}\right)}-\frac{\gamma+n_{3}^{\infty}}{\gamma+n_{3}\left(t_{s}\right)} \\
= & \frac{\gamma\left(\left(\frac{\gamma+n_{3}^{\infty}}{\gamma+n_{3}\left(t_{s}\right)}\right)^{\delta}\right)\left(\frac{1}{\gamma+n_{3}\left(t_{s}\right)}\right)}{1+\gamma\left(\left(\frac{\gamma+n_{3}^{\infty}}{\gamma+n_{3}\left(t_{f}\right)}\right)^{\delta-1}\left(\frac{1}{\gamma+n_{3}\left(t_{f}\right)}\right)+\left(\frac{\gamma+n_{3}^{\infty}}{\gamma+n_{3}\left(t_{s}\right)}\right)^{\delta-1}\left(\frac{1}{\gamma+n_{3}\left(t_{s}\right)}\right)\right)}-\frac{\gamma+n_{3}^{\infty}}{\gamma+n_{3}\left(t_{s}\right)} \\
= & \frac{-\left(\gamma+n_{3}^{\infty}\right)-\gamma\left(\frac{\gamma+n_{3}^{\infty}}{\gamma+n_{3}\left(t_{f}\right)}\right)^{\delta}}{\left(1+\gamma\left(\left(\frac{\gamma+n_{3}^{\infty}}{\gamma+n_{3}\left(t_{f}\right)}\right)^{\delta-1}\left(\frac{1}{\gamma+n_{3}\left(t_{f}\right)}\right)+\left(\frac{\gamma+n_{3}^{\infty}}{\gamma+n_{3}\left(t_{s}\right)}\right)^{\delta-1}\left(\frac{1}{\gamma+n_{3}\left(t_{s}\right)}\right)\right)\right)\left(\gamma+n_{3}\left(t_{s}\right)\right)}<0,
\end{aligned}
$$

so that $\frac{\partial n_{1}^{\infty}}{\partial n_{3}\left(t_{s}\right)}<0$. On the other hand,

$$
\frac{\partial n_{1}^{\infty}}{\partial n_{3}\left(t_{f}\right)}=\gamma\left(\left(\frac{\gamma+n_{3}^{\infty}}{\gamma+n_{3}\left(t_{s}\right)}\right)^{\delta-1}\left(\frac{1}{\gamma+n_{3}\left(t_{s}\right)}\right)\right) \frac{\partial n_{3}^{\infty}}{\partial n_{3}\left(t_{f}\right)}>0
$$

Similarly, since $n_{1}^{\infty}$ turns into $n_{2}^{\infty}$ upon exchange of $n_{3}\left(t_{f}\right)$ and $n_{3}\left(t_{s}\right), \frac{\partial n_{2}^{\infty}}{\partial n_{3}\left(t_{s}\right)}>0$ and $\frac{\partial n_{2}^{\infty}}{\partial n_{3}\left(t_{f}\right)}<0$. Now, consider a symmetric imitation setting, $\delta=1$. Noting that since $n_{3}^{\infty}$ can be re-expressed as

$$
\frac{\left.(3 \gamma+1)\left(\gamma+n_{3}\left(t_{f}\right)\right)\right)\left(\gamma+n_{3}\left(t_{s}\right)\right)}{\left(\gamma+n_{3}\left(t_{f}\right)\right)\left(\gamma+n_{3}\left(t_{s}\right)\right)+\gamma\left(2 \gamma+n_{3}\left(t_{s}\right)+n_{3}\left(t_{f}\right)\right)}-\gamma=\frac{3 \gamma+1}{1+\frac{\gamma}{\gamma+n_{3}\left(t_{f}\right)}+\frac{\gamma}{\gamma+n_{3}\left(t_{s}\right)}}-\gamma
$$


it is a monotone increasing function of both $n_{3}\left(t_{f}\right)$ and $n_{3}\left(t_{s}\right)$. On the other hand, for fixed values of $n_{3}\left(t_{f}\right)$ and $n_{3}\left(t_{s}\right)$,

$$
\frac{\partial n_{3}^{\infty}}{\partial \gamma}=\frac{3\left(3-\frac{n_{3}\left(t_{f}\right)}{\gamma+n_{3}\left(t_{f}\right)}-\frac{n_{3}\left(t_{s}\right)}{\gamma+n_{3}\left(t_{s}\right)}\right)-(3 \gamma+1)\left(\frac{n_{3}\left(t_{f}\right)}{\left(\gamma+n_{3}\left(t_{f}\right)\right)^{2}}+\frac{n_{3}\left(t_{s}\right)}{\left(\gamma+n_{3}\left(t_{s}\right)\right)^{2}}\right)}{\left(3-\frac{n_{3}\left(t_{f}\right)}{\gamma+n_{3}\left(t_{f}\right)}-\frac{n_{3}\left(t_{s}\right)}{\gamma+n_{3}\left(t_{s}\right)}\right)^{2}}-1 .
$$

This expression is negative if and only if

$$
\left(\frac{n_{3}\left(t_{f}\right)}{\gamma+n_{3}\left(t_{f}\right)}+\frac{n_{3}\left(t_{s}\right)}{\gamma+n_{3}\left(t_{s}\right)}\right)\left(3-\frac{n_{3}\left(t_{f}\right)}{\gamma+n_{3}\left(t_{f}\right)}-\frac{n_{3}\left(t_{s}\right)}{\gamma+n_{3}\left(t_{s}\right)}\right)<(3 \gamma+1)\left(\frac{n_{3}\left(t_{f}\right)}{\left(\gamma+n_{3}\left(t_{f}\right)\right)^{2}}+\frac{n_{3}\left(t_{s}\right)}{\left(\gamma+n_{3}\left(t_{s}\right)\right)^{2}}\right)
$$

which is equivalent to

$2<\left(\frac{(2 \gamma+1)}{\gamma+n_{3}\left(t_{f}\right)}+\frac{(2 \gamma+1)}{\gamma+n_{3}\left(t_{s}\right)}\right)+\frac{2 \gamma^{2}}{\left(\gamma+n_{3}\left(t_{f}\right)\right)\left(\gamma+n_{3}\left(t_{s}\right)\right)}-\left(\frac{(2 \gamma+1) \gamma}{\left(\gamma+n_{3}\left(t_{f}\right)\right)^{2}}+\frac{(2 \gamma+1) \gamma}{\left(\gamma+n_{3}\left(t_{s}\right)\right)^{2}}\right)$.

Note that for a given value of $n_{3}\left(t_{f}\right)$, the market share value $n_{3}\left(t_{s}\right)$ is greater or equal to $n_{3}\left(t_{f}\right)$ but, as follows from (29) and (49), it cannot exceed $\widehat{n}_{3}=\frac{\gamma+(1+\gamma) n_{3}\left(t_{f}\right)}{2 \gamma+n_{3}\left(t_{f}\right)}$. In other words, the value of $z_{s}=\frac{1}{\gamma+n_{3}\left(t_{s}\right)}$ belongs to the interval $\left[\frac{1}{2 \gamma+1}\left(1+\frac{\gamma}{\gamma+n_{3}\left(t_{f}\right)}\right), \frac{1}{\gamma+n_{3}\left(t_{f}\right)}\right]$. Then, for given value of $n_{3}\left(t_{f}\right)$, we only need to check validity of (A99) at the boundary points of this interval. In particular, for $z_{s}=\frac{1}{2 \gamma+1}\left(1+\frac{\gamma}{\gamma+n_{3}\left(t_{f}\right)}\right),($ A99) is equivalent to

$$
\frac{3 \gamma+1}{2 \gamma+1}<\frac{(2 \gamma+1)(\gamma+1)}{\gamma+n_{3}\left(t_{f}\right)}+\frac{\gamma^{2}+\left(1+2 \gamma^{3}\right)(1+2 \gamma)^{2}}{(1+2 \gamma)^{2}\left(\gamma+n_{3}\left(t_{f}\right)\right)^{2}}
$$

which is trivially satisfied for $n_{3}\left(t_{f}\right)=1$, and, therefore, for any $n_{3}\left(t_{f}\right) \in(0,1)$. Further, for $z_{s}=$ $\frac{1}{\gamma+n_{3}\left(t_{f}\right)},($ A99) is equivalent to

$$
1<\frac{2 \gamma+1}{\gamma+n_{3}\left(t_{f}\right)}-\frac{\gamma(\gamma+1)}{\left(\gamma+n_{3}\left(t_{f}\right)\right)^{2}}
$$

which, in turn, is trivially satisfied for any $n_{3}\left(t_{f}\right) \in(0,1)$.

\section{Proof of Proposition 7}

Adding (54) and (55), we have a Bass-like equation for $m^{\circ}(t)=m_{1}^{\circ}(t)+m_{2}^{\circ}(t)$ for $t \geq T_{F}$ :

$$
\frac{d m^{\circ}}{d t}=\left(2 p_{\mathrm{o}}+\frac{q_{\mathrm{o}}}{\mathcal{M}} m^{\mathrm{o}}\right)\left(\mathcal{M}-m^{\circ}\right)
$$


with the initial condition

$$
m^{\mathrm{o}}\left(T_{F}\right)=m_{1}^{\mathrm{o}}\left(T_{F}\right)+m_{1}^{\mathrm{o}}\left(T_{F}\right) .
$$

Defining, for $\bar{t}=t-T_{F}$,

$$
\bar{m}^{\mathrm{o}}(\bar{t})=m^{\mathrm{o}}(t)-m^{\mathrm{o}}\left(T_{F}\right),
$$

we note that (A102)-(A103) is equivalent to

$$
\frac{d m^{\mathrm{o}}}{d \bar{t}}=\left(2 p_{\mathrm{o}}+\frac{q_{\mathrm{o}}}{\mathcal{M}} m^{\mathrm{o}}\left(T_{F}\right)+\frac{q_{\mathrm{o}}}{\mathcal{M}} \bar{m}^{\mathrm{o}}\right)\left(\mathcal{M}-m^{\mathrm{o}}\left(T_{F}\right)-\bar{m}^{\circ}\right),
$$

with the initial condition

$$
\bar{m}^{\circ}(\bar{t}=0)=0 .
$$

Solution to (A105)-(A106) is given by

$$
\bar{m}^{\mathrm{o}}(\bar{t})=\left(\mathcal{M}-m^{\mathrm{o}}\left(T_{F}\right)\right)\left(1-\frac{\left(\frac{\frac{q_{\mathrm{O}}}{\mathcal{M}}\left(\mathcal{M}-m^{\circ}\left(T_{F}\right)\right)}{2 p_{\mathrm{o}}+\frac{q_{\mathrm{o}}}{\mathcal{M}} m^{\circ}\left(T_{F}\right)}+1\right) e^{-\left(2 p_{\mathrm{o}}+\frac{q_{\mathrm{o}}}{\mathcal{M}} m^{\mathrm{o}}\left(T_{F}\right)+\frac{q_{\mathrm{o}}}{\mathcal{M}}\left(\mathcal{M}-m^{\circ}\left(T_{F}\right)\right)\right) \bar{t}}}{1+\left(\frac{q_{\mathrm{O}}\left(\mathcal{M}-m^{\circ}\left(T_{F}\right)\right)}{2 p_{\mathrm{o}}+\frac{q_{\mathrm{o}}}{\mathcal{M}} m^{\circ}\left(T_{F}\right)}\right) e^{-\left(2 p_{\mathrm{o}}+\frac{q_{\mathrm{o}}}{\mathcal{M}} m^{\circ}\left(T_{F}\right)+\frac{q_{\mathrm{o}}}{\mathcal{M}}\left(\mathcal{M}-m^{\circ}\left(T_{F}\right)\right)\right) \bar{t}}}\right),
$$

resulting in

$m^{\mathrm{o}}(t)=m^{\mathrm{o}}\left(T_{F}\right)+\left(\mathcal{M}-m^{\mathrm{o}}\left(T_{F}\right)\right)\left(1-\frac{\left(\frac{\frac{q_{\mathrm{o}}}{\mathcal{M}}\left(\mathcal{M}-m^{\circ}\left(T_{F}\right)\right)}{2 p_{\mathrm{o}}+\frac{q_{\mathrm{o}}}{\mathcal{M}} m^{\circ}\left(T_{F}\right)}+1\right) e^{-\left(2 p_{\mathrm{o}}+\frac{q_{\mathrm{o}}}{\mathcal{M}} m^{\circ}\left(T_{F}\right)+\frac{q_{\mathrm{o}}}{\mathcal{M}}\left(\mathcal{M}-m^{\circ}\left(T_{F}\right)\right)\right)\left(t-T_{F}\right)}}{1+\left(\frac{\frac{q_{\mathrm{O}}}{\mathcal{M}}\left(\mathcal{M}-m^{\circ}\left(T_{F}\right)\right)}{2 p_{\mathrm{o}}+\frac{q_{\mathrm{o}}}{\mathcal{M}} m^{\circ}\left(T_{F}\right)}\right) e^{-\left(2 p_{\mathrm{o}}+\frac{q_{\mathrm{o}}}{\mathcal{M}} m^{\circ}\left(T_{F}\right)+\frac{q_{\mathrm{o}}}{\mathcal{M}}\left(\mathcal{M}-m^{\circ}\left(T_{F}\right)\right)\right)\left(t-T_{F}\right)}}\right)$,

for $t \geq T_{F}$. Then, (54) is equivalent to

$$
\frac{d m_{1}^{\mathrm{o}}}{d t}=B(t)+m_{1}^{\mathrm{o}}(t) A(t)
$$

where

$$
\begin{gathered}
B(t)=p_{\mathrm{o}}\left(\mathcal{M}-m^{\mathrm{o}}(t)\right)+p_{\mathrm{oc}} m^{\circ}(t), \\
A(t)=\frac{q_{\mathrm{o}}}{\mathcal{M}}\left(\mathcal{M}-m^{\mathrm{o}}(t)\right)-2 p_{\mathrm{oc}},
\end{gathered}
$$

and $m^{\circ}(t)$ is given by (A108). Solution to (A109) for $t \geq T_{f}$ with the initial condition $m_{1}^{\circ}\left(T_{F}\right)$ is given by

$$
m_{1}^{\circ}(t)=e^{\int_{T_{F}}^{t} A(\tau) d \tau}\left(m_{1}^{\mathrm{o}}\left(T_{F}\right)+\int_{T_{F}}^{t} B(u) d u e^{-\int_{T_{F}}^{u} A(v) d v}\right) .
$$


Consider

$$
\begin{aligned}
& \int_{T_{F}}^{t} A(\tau) d \tau \\
= & \frac{q_{\mathrm{o}}}{\mathcal{M}}\left(\mathcal{M}-m^{\mathrm{o}}\left(T_{F}\right)\right) \int_{T_{F}}^{t} d \tau\left(\frac{\left(\frac{\frac{q_{\mathrm{o}}}{\mathcal{M}}\left(\mathcal{M}-m^{\mathrm{o}}\left(T_{F}\right)\right)}{2 p_{\mathrm{o}}+\frac{q_{\mathrm{o}}}{\mathcal{M}} m^{\circ}\left(T_{F}\right)}+1\right) e^{-\left(2 p_{\mathrm{o}}+\frac{q_{\mathrm{o}}}{\mathcal{M}} m^{\circ}\left(T_{F}\right)+\frac{q_{\mathrm{o}}}{\mathcal{M}}\left(\mathcal{M}-m^{\mathrm{o}}\left(T_{F}\right)\right)\right)\left(\tau-T_{F}\right)}}{1+\left(\frac{q_{\mathrm{O}}\left(\mathcal{M}-m^{\circ}\left(T_{F}\right)\right)}{2 p_{\mathrm{o}}+\frac{q_{\mathrm{o}}}{\mathcal{M}} m^{\circ}\left(T_{F}\right)}\right) e^{-\left(2 p_{\mathrm{o}}+\frac{q_{\mathrm{o}}}{\mathcal{M}} m^{\circ}\left(T_{F}\right)+\frac{q_{\mathrm{o}}}{\mathcal{M}}\left(\mathcal{M}-m^{\circ}\left(T_{F}\right)\right)\left(\tau-T_{F}\right)\right.}}\right) \\
- & 2 p_{\mathrm{oc}}\left(t-T_{F}\right) .
\end{aligned}
$$

Note that

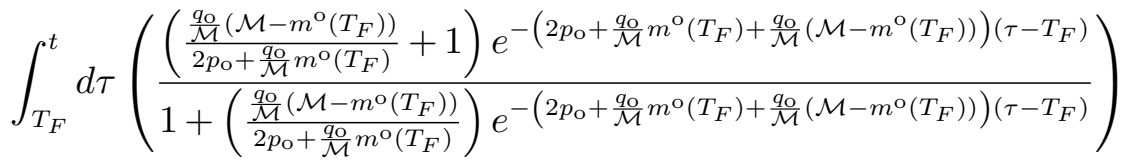

$$
\begin{aligned}
& =\left(\frac{1}{2 p_{\mathrm{o}}+\frac{q_{\mathrm{o}}}{\mathcal{M}} m^{\circ}\left(T_{F}\right)+\frac{q_{\mathrm{o}}}{\mathcal{M}}\left(\mathcal{M}-m^{\circ}\left(T_{F}\right)\right)}\right) \\
& \times \ln \left(\frac{1+\left(\frac{\frac{q_{\mathrm{O}}}{\mathcal{M}}\left(\mathcal{M}-m^{\circ}\left(T_{F}\right)\right)}{2 p_{\mathrm{o}}+\frac{q_{\mathrm{o}}}{\mathcal{M}} m^{\circ}\left(T_{F}\right)}\right)}{1+\left(\frac{q_{\mathrm{O}}\left(\mathcal{M}-m^{\circ}\left(T_{F}\right)\right)}{2 p_{\mathrm{o}}+\frac{q_{\mathrm{o}}}{\mathcal{M}} m^{\circ}\left(T_{F}\right)}\right) e^{-\left(2 p_{\mathrm{o}}+\frac{q_{\mathrm{o}}}{\mathcal{M}} m^{\circ}\left(T_{F}\right)+\frac{q_{\mathrm{o}}}{\mathcal{M}}\left(\mathcal{M}-m^{\circ}\left(T_{F}\right)\right)\right)\left(t-T_{F}\right)}}\right),
\end{aligned}
$$

so that

$$
\begin{aligned}
& e^{\int_{T_{F}}^{t} A(\tau) d \tau} \\
= & e^{-\left(2 p_{\mathrm{oc}}\left(t-T_{F}\right)\right)} \\
\times & \left(\frac{1+\left(\frac{\frac{q_{\mathrm{o}}}{\mathcal{M}}\left(\mathcal{M}-m^{\circ}\left(T_{F}\right)\right)}{2 p_{\mathrm{o}}+\frac{q_{\mathrm{o}}}{\mathcal{M}} m^{\circ}\left(T_{F}\right)}\right)}{1+\left(\frac{\frac{q_{\mathrm{o}}}{\mathcal{M}}\left(\mathcal{M}-m^{\circ}\left(T_{F}\right)\right)}{2 p_{\mathrm{o}}+\frac{q_{\mathrm{o}}}{\mathcal{M}} m^{\circ}\left(T_{F}\right)}\right) e^{-\left(2 p_{\mathrm{o}}+\frac{q_{\mathrm{o}}}{\mathcal{M}} m^{\circ}\left(T_{F}\right)+\frac{q_{\mathrm{o}}}{\mathcal{M}}\left(\mathcal{M}-m^{\circ}\left(T_{F}\right)\right)\right)\left(t-T_{F}\right)}}\right)^{\frac{1}{2 p_{\mathrm{o}}+\frac{q_{\mathrm{o}}}{\mathcal{M}} m^{\circ}\left(T_{F}\right)+\frac{q_{\mathrm{o}}}{\mathcal{M}}\left(\mathcal{M}-m^{\circ}\left(T_{F}\right)\right)}} \\
= & e^{-2 p_{\mathrm{oc}}\left(t-T_{F}\right)}\left(\frac{1+\left(\frac{q_{\mathrm{O}}\left(\mathcal{M}-m^{\circ}\left(T_{F}\right)\right)}{2 p_{\mathrm{o}}+\frac{q_{\mathrm{o}}}{\mathcal{M}} m^{\circ}\left(T_{F}\right)}\right)}{1+\left(\frac{\frac{q_{\mathrm{O}}}{\mathcal{M}}\left(\mathcal{M}-m^{\circ}\left(T_{F}\right)\right)}{2 p_{\mathrm{o}}+\frac{q_{\mathrm{o}}}{\mathcal{M}} m^{\circ}\left(T_{F}\right)}\right) e^{-\left(2 p_{\mathrm{o}}+q_{\mathrm{o}}\right)\left(t-T_{F}\right)}}\right)^{\frac{1}{2 p_{\mathrm{o}}+q_{\mathrm{o}}}}
\end{aligned}
$$

Next,

$$
\begin{aligned}
& \int_{T_{F}}^{t} B(u) d u e^{-\int_{T_{F}}^{u} A(v) d v}=\int_{T_{F}}^{t} B(u) d u\left(e^{2 p_{\mathrm{oc}}\left(u-T_{F}\right)}\right)\left(\frac{1+\left(\frac{\frac{q_{\mathrm{o}}}{\mathcal{M}\left(\mathcal{M}-m_{\mathrm{o}}^{\circ}\left(T_{F}\right)\right)}}{2 p_{\mathrm{o}}+\frac{q_{\mathrm{o}}}{\mathcal{M}} m^{\circ}\left(T_{F}\right)}\right) e^{-\left(2 p_{\mathrm{o}}+q_{\mathrm{o}}\right)\left(u-T_{F}\right)}}{1+\left(\frac{q_{\mathrm{o}}\left(\mathcal{M}-m^{\circ}\left(T_{F}\right)\right)}{2 p_{\mathrm{o}}+\frac{q_{\mathrm{o}}}{\mathcal{M}} m^{\circ}\left(T_{F}\right)}\right)}\right)^{\frac{1}{2 p_{\mathrm{o}}+q_{\mathrm{o}}}} \\
= & \int_{T_{F}}^{t} d u\left(e^{2 p_{\mathrm{oc}}\left(u-T_{F}\right)}\right)\left(\frac{1+\left(\frac{q_{\mathrm{O}}\left(\mathcal{M}-m^{\circ}\left(T_{F}\right)\right)}{2 p_{\mathrm{o}}+\frac{q_{\mathrm{o}}}{\mathcal{M}} m^{\circ}\left(T_{F}\right)}\right) e^{-\left(2 p_{\mathrm{o}}+q_{\mathrm{o}}\right)\left(u-T_{F}\right)}}{1+\left(\frac{\frac{q_{\mathrm{o}}}{\mathcal{M}}\left(\mathcal{M}-m^{\circ}\left(T_{F}\right)\right)}{2 p_{\mathrm{o}}+\frac{q_{\mathrm{o}}}{\mathcal{M}} m^{\mathrm{o}}\left(T_{F}\right)}\right)}\right)^{\frac{1}{2 p_{\mathrm{o}}+q_{\mathrm{o}}}}\left(p_{\mathrm{o}} \mathcal{M}+\left(p_{\mathrm{oc}}-p_{\mathrm{o}}\right) m^{\mathrm{o}}(u)\right) \\
= & \left(\frac{1}{1+\left(\frac{\frac{q_{\mathrm{O}}}{\mathcal{M}}\left(\mathcal{M}-m^{\circ}\left(T_{F}\right)\right)}{2 p_{\mathrm{o}}+\frac{q_{\mathrm{o}}}{\mathcal{M}} m^{\circ}\left(T_{F}\right)}\right)}\right)^{\frac{1}{2 p_{\mathrm{o}}+q_{\mathrm{o}}}} \bar{I}(t),
\end{aligned}
$$


where

$$
\begin{aligned}
& \bar{I}(t)=\int_{0}^{t-T_{F}} d u e^{2 p_{\mathrm{oc}} u}\left(1+\left(\frac{\frac{q_{\mathrm{o}}}{\mathcal{M}}\left(\mathcal{M}-m^{\mathrm{o}}\left(T_{F}\right)\right)}{2 p_{\mathrm{o}}+\frac{q_{\mathrm{o}}}{\mathcal{M}} m^{\mathrm{o}}\left(T_{F}\right)}\right) e^{-\left(2 p_{\mathrm{o}}+q_{\mathrm{o}}\right) u}\right)^{\frac{1}{2 p_{\mathrm{o}}+q_{\mathrm{o}}}}\left(p_{\mathrm{o}} \mathcal{M}+\left(p_{\mathrm{oc}}-p_{\mathrm{o}}\right) m^{\mathrm{o}}\left(u+T_{F}\right)\right) \\
= & \left.\left.\left.\int_{0}^{t-T_{F}} d u e^{2 p_{\mathrm{oc}} u}\left(1+\left(\frac{\frac{q_{\mathrm{o}}}{\mathcal{M}}\left(\mathcal{M}-m^{\mathrm{o}}\left(T_{F}\right)\right)}{2 p_{\mathrm{o}}+\frac{q_{\mathrm{o}}}{\mathcal{M}} m^{\mathrm{o}}\left(T_{F}\right)}\right) e^{-\left(2 p_{\mathrm{o}}+q_{\mathrm{o}}\right) u}\right)^{\frac{1}{2 p_{\mathrm{o}}+q_{\mathrm{o}}}}\right)\right)\right) \\
\times & \left(p_{\mathrm{o}} \mathcal{M}+\left(p_{\mathrm{oc}}-p_{\mathrm{o}}\right)\left(m^{\mathrm{o}}\left(T_{F}\right)+\left(\frac{\mathcal{M}-m^{\mathrm{o}}\left(T_{F}\right)}{1+\left(\frac{q_{\mathrm{O}}\left(\mathcal{M}-m^{\circ}\left(T_{F}\right)\right)}{2 p_{\mathrm{o}}+\frac{q_{\mathrm{o}}}{\mathcal{M}} m^{\circ}\left(T_{F}\right)}\right) e^{-\left(2 p_{\mathrm{o}}+q_{\mathrm{o}}\right) u}}\right)\right)\right. \\
= & \left(p_{\mathrm{o}} \mathcal{M}+\left(p_{\mathrm{oc}}-p_{\mathrm{o}}\right) m^{\mathrm{o}}\left(T_{F}\right)\right) \bar{I}_{1}(t)+\left(p_{\mathrm{oc}}-p_{\mathrm{o}}\right)\left(\mathcal{M}-m^{\mathrm{o}}\left(T_{F}\right)\right) \bar{I}_{2}(t),
\end{aligned}
$$

with

$$
\begin{aligned}
& \bar{I}_{1}(t)=\int_{0}^{t-T_{F}} d u e^{2 p_{\mathrm{oc}} u}\left(1+\left(\frac{\frac{q_{\mathrm{o}}}{\mathcal{M}}\left(\mathcal{M}-m^{\mathrm{o}}\left(T_{F}\right)\right)}{2 p_{\mathrm{o}}+\frac{q_{\mathrm{o}}}{\mathcal{M}} m^{\mathrm{o}}\left(T_{F}\right)}\right) e^{-\left(2 p_{\mathrm{o}}+q_{\mathrm{o}}\right) u}\right)^{\frac{1}{2 p_{\mathrm{o}}+q_{\mathrm{o}}}} \\
= & \frac{1}{2 p_{\mathrm{oc}}} \int_{1}^{e^{2 p_{\mathrm{oc}}\left(t-T_{F}\right)}} d x\left(1+\left(\frac{\frac{q_{\mathrm{o}}}{\mathcal{M}}\left(\mathcal{M}-m^{\mathrm{o}}\left(T_{F}\right)\right)}{2 p_{\mathrm{o}}+\frac{q_{\mathrm{o}}}{\mathcal{M}} m^{\mathrm{o}}\left(T_{F}\right)}\right) x^{-\left(\frac{2 p_{\mathrm{o}}+q_{\mathrm{o}}}{2 p_{\mathrm{oc}}}\right)}\right)^{\frac{1}{2 p_{\mathrm{o}}+q_{\mathrm{o}}}} \\
= & \frac{1}{2 p_{\mathrm{oc}}}\left(e^{2 p_{\mathrm{oc}}\left(t-T_{F}\right)}{ }_{2} F_{1}\left(\frac{2 p_{\mathrm{oc}}}{2 p_{\mathrm{o}}+q_{\mathrm{o}}},-\frac{1}{2 p_{\mathrm{o}}+q_{\mathrm{o}}} ; 1+\frac{2 p_{\mathrm{oc}}}{2 p_{\mathrm{o}}+q_{\mathrm{o}}} ;\left(-\frac{\frac{q_{\mathrm{o}}}{\mathcal{M}}\left(\mathcal{M}-m^{\mathrm{o}}\left(T_{F}\right)\right)}{2 p_{\mathrm{o}}+\frac{q_{\mathrm{o}}}{\mathcal{M}} m^{\mathrm{o}}\left(T_{F}\right)}\right)\left(e^{-\left(2 p_{\mathrm{o}+q_{\mathrm{o}}}\right)\left(t-T_{F}\right)}\right)\right)\right. \\
& \left.-{ }_{2} F_{1}\left(\frac{2 p_{\mathrm{oc}}}{2 p_{\mathrm{o}}+q_{\mathrm{o}}},-\frac{1}{2 p_{\mathrm{o}}+q_{\mathrm{o}}} ; 1+\frac{2 p_{\mathrm{oc}}}{2 p_{\mathrm{o}}+q_{\mathrm{o}}} ;\left(-\frac{\frac{q_{\mathrm{o}}}{\mathcal{M}}\left(\mathcal{M}-m^{\mathrm{o}}\left(T_{F}\right)\right)}{2 p_{\mathrm{o}}+\frac{q_{\mathrm{o}}}{\mathcal{M}} m^{\mathrm{o}}\left(T_{F}\right)}\right)\right)\right),
\end{aligned}
$$

and

$$
\begin{aligned}
& \bar{I}_{2}(t)=\int_{0}^{t-T_{F}} d u e^{2 p_{\mathrm{oc}} u}\left(1+\left(\frac{\frac{q_{\mathrm{o}}}{\mathcal{M}}\left(\mathcal{M}-m^{\mathrm{o}}\left(T_{F}\right)\right)}{2 p_{\mathrm{o}}+\frac{q_{\mathrm{o}}}{\mathcal{M}} m^{\mathrm{o}}\left(T_{F}\right)}\right) e^{-\left(2 p_{\mathrm{o}}+q_{\mathrm{o}}\right) u}\right)^{\frac{1}{2 p_{\mathrm{o}}+q_{\mathrm{o}}}-1} \\
= & \frac{1}{2 p_{\mathrm{oc}}}\left(e^{2 p_{\mathrm{oc}}\left(t-T_{F}\right)}{ }_{2} F_{1}\left(\frac{2 p_{\mathrm{oc}}}{2 p_{\mathrm{o}}+q_{\mathrm{o}}}, 1-\frac{1}{2 p_{\mathrm{o}}+q_{\mathrm{o}}} ; 1+\frac{2 p_{\mathrm{oc}}}{2 p_{\mathrm{o}}+q_{\mathrm{o}}} ;\left(-\frac{\frac{q_{\mathrm{o}}}{\mathcal{M}}\left(\mathcal{M}-m^{\mathrm{o}}\left(T_{F}\right)\right)}{2 p_{\mathrm{o}}+\frac{q_{\mathrm{o}}}{\mathcal{M}} m^{\mathrm{o}}\left(T_{F}\right)}\right)\left(e^{-\left(2 p_{\mathrm{o}}+q_{\mathrm{o}}\right)\left(t-T_{F}\right)}\right)\right)\right. \\
& \left.-{ }_{2} F_{1}\left(\frac{2 p_{\mathrm{oc}}}{2 p_{\mathrm{o}}+q_{\mathrm{o}}}, 1-\frac{1}{2 p_{\mathrm{o}}+q_{\mathrm{o}}} ; 1+\frac{2 p_{\mathrm{oc}}}{2 p_{\mathrm{o}}+q_{\mathrm{o}}} ;\left(-\frac{\frac{q_{\mathrm{o}}}{\mathcal{M}}\left(\mathcal{M}-m^{\mathrm{o}}\left(T_{F}\right)\right)}{2 p_{\mathrm{o}}+\frac{q_{\mathrm{o}}}{\mathcal{M}} m^{\mathrm{o}}\left(T_{F}\right)}\right)\right)\right) .
\end{aligned}
$$

Thus, (A112) is equivalent to

$$
\begin{aligned}
& m_{1}^{\mathrm{o}}(t)=e^{\int_{T_{F}}^{t} A(\tau) d \tau}\left(m_{1}^{\mathrm{o}}\left(T_{F}\right)+\int_{T_{F}}^{t} B(u) d u e^{-\int_{T_{F}}^{u} A(v) d v}\right) \\
& =e^{-2 p_{\mathrm{oc}}\left(t-T_{F}\right)}\left(\frac{1+\left(\frac{\frac{q_{\mathrm{o}}}{\mathcal{M}}\left(\mathcal{M}-m^{\mathrm{o}}\left(T_{F}\right)\right)}{2 p_{\mathrm{o}}+\frac{q_{\mathrm{O}}}{\mathcal{M}} m^{\circ}\left(T_{F}\right)}\right)}{1+\left(\frac{\frac{q_{\mathrm{O}}}{\mathcal{M}}\left(\mathcal{M}-m^{\circ}\left(T_{F}\right)\right)}{2 p_{\mathrm{o}}+\frac{q_{\mathrm{o}}}{\mathcal{M}} m^{\circ}\left(T_{F}\right)}\right) e^{-\left(2 p_{\mathrm{o}}+q_{\mathrm{o}}\right)\left(t-T_{F}\right)}}\right)^{\frac{1}{2 p_{\mathrm{o}}+q_{\mathrm{o}}}} \\
& \times\left(m_{1}^{\circ}\left(T_{F}\right)\right. \\
& \left.+\left(\frac{1}{1+\left(\frac{q_{\mathrm{O}}\left(\mathcal{M}-m^{\circ}\left(T_{F}\right)\right)}{2 p_{\mathrm{o}}+\frac{q_{\mathrm{o}}}{\mathcal{M}} m^{\circ}\left(T_{F}\right)}\right)}\right)^{\frac{1}{2 p_{\mathrm{o}}+q_{\mathrm{o}}}}\left(\left(p_{\mathrm{o}} \mathcal{M}+\left(p_{\mathrm{oc}}-p_{\mathrm{o}}\right) m^{\mathrm{o}}\left(T_{F}\right)\right) \bar{I}_{1}(t)+\left(p_{\mathrm{oc}}-p_{\mathrm{o}}\right)\left(\mathcal{M}-m^{\mathrm{o}}\left(T_{F}\right)\right) \bar{I}_{2}(t)\right)\right) .
\end{aligned}
$$


Using the same approach, we obtain

$$
\begin{aligned}
& m_{2}^{\mathrm{o}}(t)=e^{\int_{T_{F}}^{t} A(\tau) d \tau}\left(m_{2}^{\mathrm{o}}\left(T_{F}\right)+\int_{T_{F}}^{t} B(u) d u e^{-\int_{T_{F}}^{u} A(v) d v}\right) \\
& =e^{-2 p_{\mathrm{oc}}\left(t-T_{F}\right)}\left(\frac{1+\left(\frac{\frac{q_{\mathrm{O}}}{\mathcal{M}}\left(\mathcal{M}-m^{\circ}\left(T_{F}\right)\right)}{2 p_{\mathrm{o}}+\frac{q_{\mathrm{O}}}{\mathcal{M}} m^{\circ}\left(T_{F}\right)}\right)}{1+\left(\frac{q_{\mathrm{O}}\left(\mathcal{M}-m^{\circ}\left(T_{F}\right)\right)}{2 p_{\mathrm{o}}+\frac{q_{\mathrm{o}}}{\mathcal{M}} m^{\circ}\left(T_{F}\right)}\right) e^{-\left(2 p_{\mathrm{o}}+q_{\mathrm{o}}\right)\left(t-T_{F}\right)}}\right)^{\frac{1}{2 p_{\mathrm{o}}+q_{\mathrm{o}}}} \\
& \times\left(m_{2}^{\circ}\left(T_{F}\right)\right. \\
& \left.+\left(\frac{1}{1+\left(\frac{q_{\mathrm{o}}\left(\mathcal{M}-m^{\circ}\left(T_{F}\right)\right)}{2 p_{\mathrm{o}}+\frac{q_{\mathrm{o}}}{\mathcal{M}} m^{\circ}\left(T_{F}\right)}\right)}\right)^{\frac{1}{2 p_{\mathrm{o}}+q_{\mathrm{o}}}}\left(\left(p_{\mathrm{o}} \mathcal{M}+\left(p_{\mathrm{oc}}-p_{\mathrm{o}}\right) m^{\mathrm{o}}\left(T_{F}\right)\right) \bar{I}_{1}(t)+\left(p_{\mathrm{oc}}-p_{\mathrm{o}}\right)\left(\mathcal{M}-m^{\mathrm{o}}\left(T_{F}\right)\right) \bar{I}_{2}(t)\right)\right) .
\end{aligned}
$$

\section{Proof of Proposition 8}

a) Consider a setting where the innovation succeeds. In this setting, the terminal customer base values can be obtained by equating the right-hand sides in (59)-(63) to 0 , in the limit of $t \rightarrow \infty$. In particular, adding the right-hand sides of (61)-(63), we get

$$
n_{1}^{\infty}+n_{2}^{\infty}+n_{3}^{\infty}=1 .
$$

Then, the right-hand sides of (61) and (62) imply that

$$
\begin{aligned}
& p_{\mathrm{nc}}^{12} n_{2}^{\infty}+p_{\mathrm{nc}}^{13} n_{3}^{\infty}-\left(p_{\mathrm{nc}}^{21}+p_{\mathrm{nc}}^{31}\right) n_{1}^{\infty}=0, \\
& p_{\mathrm{nc}}^{21} n_{1}^{\infty}+p_{\mathrm{nc}}^{23} n_{3}^{\infty}-\left(p_{\mathrm{nc}}^{12}+p_{\mathrm{nc}}^{32}\right) n_{2}^{\infty}=0 .
\end{aligned}
$$

Combining (A123)-(A124) with (A122), we obtain

$$
\mathbf{P}\left(\begin{array}{c}
n_{1}^{\infty} \\
n_{2}^{\infty} \\
n_{3}^{\infty}
\end{array}\right)=\left(\begin{array}{l}
1 \\
0 \\
0
\end{array}\right),
$$

where

$$
\mathbf{P}=\left(\begin{array}{ccc}
1 & 1 & 1 \\
-\left(p_{\mathrm{nc}}^{21}+p_{\mathrm{nc}}^{31}\right) & p_{\mathrm{nc}}^{12} & p_{\mathrm{nc}}^{13} \\
p_{\mathrm{nc}}^{21} & -\left(p_{\mathrm{nc}}^{12}+p_{\mathrm{nc}}^{32}\right) & p_{\mathrm{nc}}^{23}
\end{array}\right) .
$$


Then,

$$
\left(\begin{array}{c}
n_{1}^{\infty} \\
n_{2}^{\infty} \\
n_{3}^{\infty}
\end{array}\right)=\mathbf{P}^{-1}\left(\begin{array}{l}
1 \\
0 \\
0
\end{array}\right) .
$$

Note that

$$
\mathbf{P}^{-1}=\frac{1}{\operatorname{det}(\mathbf{P})}\left(\begin{array}{ccc}
p_{\mathrm{nc}}^{12} p_{\mathrm{nc}}^{23}+p_{\mathrm{nc}}^{13}\left(p_{\mathrm{nc}}^{12}+p_{\mathrm{nc}}^{32}\right) & -\left(p_{\mathrm{nc}}^{12}+p_{\mathrm{nc}}^{32}+p_{\mathrm{nc}}^{23}\right) & p_{\mathrm{nc}}^{13}-p_{\mathrm{nc}}^{12} \\
\left(p_{\mathrm{nc}}^{21}+p_{\mathrm{nc}}^{31}\right) p_{\mathrm{nc}}^{23}+p_{\mathrm{nc}}^{13} p_{\mathrm{nc}}^{21} & p_{\mathrm{nc}}^{23}-p_{\mathrm{nc}}^{21} & -\left(p_{\mathrm{nc}}^{13}+p_{\mathrm{nc}}^{21}+p_{\mathrm{nc}}^{31}\right) \\
\left(p_{\mathrm{nc}}^{21}+p_{\mathrm{nc}}^{31}\right)\left(p_{\mathrm{nc}}^{12}+p_{\mathrm{nc}}^{32}\right)-p_{\mathrm{nc}}^{21} p_{\mathrm{nc}}^{12} & \left(p_{\mathrm{nc}}^{12}+p_{\mathrm{nc}}^{32}++p_{\mathrm{nc}}^{21}\right) & \left(p_{\mathrm{nc}}^{12}+p_{\mathrm{nc}}^{21}+p_{\mathrm{nc}}^{31}\right)
\end{array}\right),
$$

where

$$
\begin{aligned}
& \operatorname{det}(\mathbf{P})=p_{\mathrm{nc}}^{12} p_{\mathrm{nc}}^{23}+p_{\mathrm{nc}}^{21} p_{\mathrm{nc}}^{13}+\left(p_{\mathrm{nc}}^{21}+p_{\mathrm{nc}}^{31}\right)\left(p_{\mathrm{nc}}^{12}+p_{\mathrm{nc}}^{32}\right)-p_{\mathrm{nc}}^{12} p_{\mathrm{nc}}^{21}+p_{\mathrm{nc}}^{23}\left(p_{\mathrm{nc}}^{21}+p_{\mathrm{nc}}^{31}\right)+p_{\mathrm{nc}}^{13}\left(p_{\mathrm{nc}}^{12}+p_{\mathrm{nc}}^{32}\right) \\
= & p_{\mathrm{nc}}^{12} p_{\mathrm{nc}}^{23}+p_{\mathrm{nc}}^{21} p_{\mathrm{nc}}^{13}+p_{\mathrm{nc}}^{32} p_{\mathrm{nc}}^{21}+p_{\mathrm{nc}}^{31}\left(p_{\mathrm{nc}}^{12}+p_{\mathrm{nc}}^{32}\right)+p_{\mathrm{nc}}^{23}\left(p_{\mathrm{nc}}^{21}+p_{\mathrm{nc}}^{31}\right)+p_{\mathrm{nc}}^{13}\left(p_{\mathrm{nc}}^{12}+p_{\mathrm{nc}}^{32}\right) \\
= & p_{\mathrm{nc}}^{12} p_{\mathrm{nc}}^{23}+p_{\mathrm{nc}}^{21} p_{\mathrm{nc}}^{13}+p_{\mathrm{nc}}^{31} p_{\mathrm{nc}}^{12}+p_{\mathrm{nc}}^{23} p_{\mathrm{nc}}^{31}+p_{\mathrm{nc}}^{13} p_{\mathrm{nc}}^{32}+p_{\mathrm{nc}}^{32} p_{\mathrm{nc}}^{21}+p_{\mathrm{nc}}^{12} p_{\mathrm{nc}}^{13}+p_{\mathrm{nc}}^{21} p_{\mathrm{nc}}^{23}+p_{\mathrm{nc}}^{31} p_{\mathrm{nc}}^{32} .
\end{aligned}
$$

Thus,

$$
\left(\begin{array}{c}
n_{1}^{\infty} \\
n_{2}^{\infty} \\
n_{3}^{\infty}
\end{array}\right)=\frac{1}{\operatorname{det}(\mathbf{P})}\left(\begin{array}{c}
p_{\mathrm{nc}}^{12} p_{\mathrm{nc}}^{23}+p_{\mathrm{nc}}^{13}\left(p_{\mathrm{nc}}^{12}+p_{\mathrm{nc}}^{32}\right) \\
\left(p_{\mathrm{nc}}^{21}+p_{\mathrm{nc}}^{31}\right) p_{\mathrm{nc}}^{23}+p_{\mathrm{nc}}^{13} p_{\mathrm{nc}}^{21} \\
\left(p_{\mathrm{nc}}^{21}+p_{\mathrm{nc}}^{31}\right)\left(p_{\mathrm{nc}}^{12}+p_{\mathrm{nc}}^{32}\right)-p_{\mathrm{nc}}^{21} p_{\mathrm{nc}}^{12}
\end{array}\right)=\frac{1}{\operatorname{det}(\mathbf{P})}\left(\begin{array}{c}
p_{\mathrm{nc}}^{12} p_{\mathrm{nc}}^{23}+p_{\mathrm{nc}}^{13} p_{\mathrm{nc}}^{32}+p_{\mathrm{nc}}^{12} p_{\mathrm{nc}}^{13} \\
p_{\mathrm{nc}}^{21} p_{\mathrm{nc}}^{13}+p_{\mathrm{nc}}^{23} p_{\mathrm{nc}}^{31}+p_{\mathrm{nc}}^{21} p_{\mathrm{nc}}^{23} \\
p_{\mathrm{nc}}^{32} p_{\mathrm{nc}}^{21}+p_{\mathrm{nc}}^{31} p_{\mathrm{nc}}^{12}+p_{\mathrm{nc}}^{31} p_{\mathrm{nc}}^{32}
\end{array}\right),
$$

which is equivalent to (64)-(66).

Now, using the limit of $t \rightarrow \infty$ for the right-hand sides of (59)-(60), and noting (A122), we get

$$
\begin{aligned}
& -\left(p_{\mathrm{o}}+q_{\mathrm{o}} o_{1}^{\infty}\right)\left(o_{1}^{\infty}+o_{2}^{\infty}\right)+p_{\mathrm{oc}}^{12} o_{2}^{\infty}-\left(p_{\mathrm{oc}}^{21}+3 p_{\mathrm{n}}+Q_{\mathrm{n}}\left(n_{1}^{\infty}+n_{2}^{\infty}\right)+q_{\mathrm{n}} n_{3}^{\infty}\right) o_{1}^{\infty}=0, \\
& -\left(p_{\mathrm{o}}+q_{\mathrm{o}} o_{2}^{\infty}\right)\left(o_{1}^{\infty}+o_{2}^{\infty}\right)+p_{\mathrm{oc}}^{21} o_{1}^{\infty}-\left(p_{\mathrm{oc}}^{12}+3 p_{\mathrm{n}}+Q_{\mathrm{n}}\left(n_{1}^{\infty}+n_{2}^{\infty}\right)+q_{\mathrm{n}} n_{3}^{\infty}\right) o_{2}^{\infty}=0 .
\end{aligned}
$$

Adding the two equations in (A131), we get

$$
-\left(2 p_{\mathrm{o}}+q_{\mathrm{o}}\left(o_{1}^{\infty}+o_{2}^{\infty}\right)\right)\left(o_{1}^{\infty}+o_{2}^{\infty}\right)-\left(3 p_{\mathrm{n}}+Q_{\mathrm{n}}\left(n_{1}^{\infty}+n_{2}^{\infty}\right)+q_{\mathrm{n}} n_{3}^{\infty}\right)\left(o_{1}^{\infty}+o_{2}^{\infty}\right)=0
$$

that results in $o_{1}^{\infty}=o_{2}^{\infty}=0$. 
b) In the setting where the innovation fails at $t=T_{F}$, for any $t>T_{F}$ the customer base dynamics is described by the modified version of (54)-(55):

$$
\begin{aligned}
\frac{d m_{1}^{\mathrm{o}}}{d t} & =\left(p_{\mathrm{o}}+\frac{q_{\mathrm{o}}}{\mathcal{M}} m_{1}^{\mathrm{o}}\right)\left(\mathcal{M}-m_{1}^{\mathrm{o}}-m_{2}^{\mathrm{o}}\right)+p_{\mathrm{oc}}^{12} m_{2}^{\mathrm{o}}-p_{\mathrm{oc}}^{21} m_{1}^{\mathrm{o}}, \\
\frac{d m_{2}^{\mathrm{o}}}{d t} & =\left(p_{\mathrm{o}}+\frac{q_{\mathrm{o}}}{\mathcal{M}} m_{2}^{\mathrm{o}}\right)\left(\mathcal{M}-m_{1}^{\mathrm{o}}-m_{2}^{\mathrm{o}}\right)+p_{\mathrm{oc}}^{21} m_{1}^{\mathrm{o}}-p_{\mathrm{oc}}^{12} m_{2}^{\mathrm{o}} .
\end{aligned}
$$

In the limit of $t \rightarrow \infty$, these result in

$$
\begin{aligned}
& \left(p_{\mathrm{o}}+q_{\mathrm{o}} o_{1}^{\infty}\right)\left(1-o_{1}^{\infty}-o_{2}^{\infty}\right)+p_{\mathrm{oc}}^{12} o_{2}^{\infty}-p_{\mathrm{oc}}^{21} o_{1}^{\infty}=0, \\
& \left(p_{\mathrm{o}}+q_{\mathrm{o}} o_{2}^{\infty}\right)\left(1-o_{1}^{\infty}-o_{2}^{\infty}\right)+p_{\mathrm{oc}}^{21} o_{1}^{\infty}-p_{\mathrm{oc}}^{12} o_{2}^{\infty}=0 .
\end{aligned}
$$

Adding the two equations in (A135), we get

$$
1-o_{1}^{\infty}-o_{2}^{\infty}=0
$$

so that the solution to (A135) is given by the combination of

$$
p_{\mathrm{oc}}^{12} o_{2}^{\infty}-p_{\mathrm{oc}}^{21} o_{1}^{\infty}=0
$$

and (A136). Then, (67)-(68) follow.

\section{Proof of Proposition 9}

We will prove the quasiconcavity of the firm's 1 profit function by showing that this function cannot have unconstrained local minima. Define $T_{c}$ as a set of local unconstrained optima of $\Pi_{1}\left(t_{1}, t_{2}\right):$

$$
T_{c}=\left(0<t<t_{\max }\left|\frac{\partial \Pi_{1}\left(t_{1}, t_{2}\right)}{\partial t_{1}}\right|_{t_{1}=t}=0\right) .
$$

The statement of the Proposition will be established if we show that

$$
\left.\frac{\partial^{2} \Pi_{1}\left(t_{1}, t_{2}\right)}{\partial\left(t_{1}\right)^{2}}\right|_{t_{1}=t^{*}} \leq 0
$$

for any $t^{*} \in T_{c}$. Consider the case where $t^{*} \geq t_{2}$ (the proof for the case of $t^{*} \leq t_{2}$ follows similar steps). Then,

$$
\left.\frac{\partial \Pi_{1}\left(t_{1}, t_{2}\right)}{\partial t_{1}}\right|_{t_{1}=t^{*}}=0 \Leftrightarrow \rho\left(\int_{t^{*}}^{+\infty} e^{-\beta t}\left(\frac{\partial o_{13}\left(t, t^{*}, t_{2}\right)}{\partial t^{*}}\right) d t\right)+\left(\int_{t^{*}}^{+\infty} e^{-\beta t}\left(\frac{\partial n_{1}\left(t, t^{*}, t_{2}\right)}{\partial t^{*}}\right) d t\right)=0,
$$


where $\rho=\frac{\theta \pi_{\mathrm{o}}}{\pi_{\mathrm{n}}}$ and

$$
\begin{aligned}
& \int_{t^{*}}^{+\infty} e^{-\beta t}\left(\frac{\partial n_{1}\left(t, t^{*}, t_{2}\right)}{\partial t^{*}}\right) d t=e^{-\beta t^{*}} \frac{d n_{3}\left(t^{*}\right)}{d t^{*}} \frac{p_{\mathrm{n}}}{p_{\mathrm{n}}+q_{\mathrm{n}} n_{3}\left(t^{*}\right)} \int_{0}^{+\infty} e^{-\beta u}\left(\frac{Q_{\mathrm{n}}}{p_{\mathrm{n}}} S\left(n_{3}(t), n_{3}\left(t^{*}\right)\right)+1\right) \\
& \times\left(\frac{n_{3}\left(t^{*}\right)-n_{3}(t)-S\left(n_{3}(t), n_{3}\left(t^{*}\right)\right)\left(2+\frac{Q_{\mathrm{n}}}{p_{\mathrm{n}}} S\left(n_{3}\left(t^{*}\right), n_{3}\left(t_{2}\right)\right)\right)}{1-S\left(n_{3}\left(t^{*}\right), n_{3}\left(t_{2}\right)\right)-n_{3}\left(t^{*}\right)}\right) d u,
\end{aligned}
$$

and

$$
\begin{aligned}
& \int_{t^{*}}^{+\infty} e^{-\beta t}\left(\frac{\partial o_{13}\left(t, t^{*}, t_{2}\right)}{\partial t^{*}}\right) d t=e^{-\beta t^{*}} \int_{0}^{+\infty} e^{-\beta u}\left(\frac{\partial o_{13}\left(t, t^{*}, t_{2}\right)}{\partial u}\right) d u \\
= & e^{-\beta t^{*}} \frac{d n_{3}\left(t^{*}\right)}{d t^{*}} \int_{0}^{+\infty} e^{-\beta u}\left(\frac{\partial o_{13}\left(t, t^{*}, t_{2}\right)}{\partial n_{3}\left(t^{*}\right)}+\frac{\partial o_{13}\left(t, t^{*}, t_{2}\right)}{\partial n_{3}(t)} \frac{\partial n_{3}(t)}{\partial n_{3}\left(t^{*}\right)}\right) d u \\
= & -\int_{t^{*}}^{+\infty} e^{-\beta t}\left(\frac{\partial n_{1}\left(t, t^{*}, t_{2}\right)}{\partial t^{*}}\right) d t e^{-\beta t^{*}}-\frac{d n_{3}\left(t^{*}\right)}{d t^{*}} \frac{p_{\mathrm{n}}}{p_{\mathrm{n}}+q_{\mathrm{n}} n_{3}\left(t^{*}\right)} \frac{1}{1-S\left(n_{3}\left(t^{*}\right), n_{3}\left(t_{2}\right)\right)-n_{3}\left(t^{*}\right)} \\
& \times \int_{0}^{+\infty} e^{-\beta u}\left[1+\frac{Q_{\mathrm{n}}}{p_{\mathrm{n}}} S\left(n_{3}(t), n_{3}\left(t_{2}\right)\right)+\frac{p_{\mathrm{n}}+q_{\mathrm{n}} n_{3}(t)}{p_{\mathrm{n}}}\right] \\
& \times\left(1-S\left(n_{3}\left(t^{*}\right), n_{3}\left(t_{2}\right)\right)-n_{3}(t)-S\left(n_{3}(t), n_{3}\left(t^{*}\right)\right)\left(2+\frac{Q_{\mathrm{n}}}{p_{\mathrm{n}}} S\left(n_{3}\left(t^{*}\right), n_{3}\left(t_{2}\right)\right)\right)\right) d u . \quad(\mathrm{A} 14
\end{aligned}
$$

The sign of the second derivative of the profit function at a local optimum depends on whether the ratio

$$
R=\frac{\int_{t^{*}}^{+\infty} e^{-\beta t}\left(\frac{\partial o_{13}\left(t, t^{*}, t_{2}\right)}{\partial t^{*}}\right) d t}{\int_{t^{*}}^{+\infty} e^{-\beta t}\left(\frac{\partial n_{1}\left(t, t^{*}, t_{2}\right)}{\partial t^{*}}\right) d t}
$$

is an increasing or a decreasing function of $t^{*}$. Using a shorthand notation $n_{3}=n_{3}(t), n_{3}^{*}=n_{3}\left(t^{*}\right)$, $n_{3}^{2}=n_{3}\left(t_{2}\right)$, we get

$$
R=-1-\frac{\int_{0}^{+\infty} e^{-\beta u}\left(1+\frac{Q_{\mathrm{n}}}{p_{\mathrm{n}}} S\left(n_{3}, n_{3}^{2}\right)+\frac{p_{\mathrm{n}}+q_{\mathrm{n}} n_{3}}{p_{\mathrm{n}}}\right)\left(1-S\left(n_{3}^{*}, n_{3}^{2}\right)-n_{3}-S\left(n_{3}, n_{3}^{*}\right)\left(2+\frac{Q_{\mathrm{n}}}{p_{\mathrm{n}}} S\left(n_{3}^{*}, n_{3}^{2}\right)\right)\right) d u}{\int_{0}^{+\infty} e^{-\beta u}\left(\frac{Q_{\mathrm{n}}}{p_{\mathrm{n}}} S\left(n_{3}, n_{3}^{*}\right)+1\right)\left(n_{3}^{*}-n_{3}-S\left(n_{3}, n_{3}^{*}\right)\left(2+\frac{Q_{\mathrm{n}}}{p_{\mathrm{n}}} S\left(n_{3}^{*}, n_{3}^{2}\right)\right)\right) d u},
$$

so that now we need to check the monotonicity of

$$
\widehat{R}=\frac{\int_{0}^{+\infty} e^{-\beta u}\left(1+\frac{Q_{\mathrm{n}}}{p_{\mathrm{n}}} S\left(n_{3}, n_{3}^{2}\right)+\frac{p_{\mathrm{n}}+q_{\mathrm{n}} n_{3}}{p_{\mathrm{n}}}\right)\left(1-S\left(n_{3}^{*}, n_{3}^{2}\right)-n_{3}-S\left(n_{3}, n_{3}^{*}\right)\left(2+\frac{Q_{\mathrm{n}}}{p_{\mathrm{n}}} S\left(n_{3}^{*}, n_{3}^{2}\right)\right)\right) d u}{\int_{0}^{+\infty} e^{-\beta u}\left(\frac{Q_{\mathrm{n}}}{p_{\mathrm{n}}} S\left(n_{3}, n_{3}^{*}\right)+1\right)\left(n_{3}^{*}-n_{3}-S\left(n_{3}, n_{3}^{*}\right)\left(2+\frac{Q_{\mathrm{n}}}{p_{\mathrm{n}}} S\left(n_{3}^{*}, n_{3}^{2}\right)\right)\right) d u}
$$




$$
\begin{aligned}
& =\frac{\left(1+\frac{Q_{\mathrm{n}}}{p_{\mathrm{n}}} S\left(n_{3}^{*}, n_{3}^{2}\right)\right) \frac{1}{p_{\mathrm{n}}} \int_{0}^{+\infty} e^{-\beta u} d S\left(n_{3}, n_{3}^{*}\right)+\frac{1}{p_{\mathrm{n}}}\left(\beta \int_{0}^{+\infty} e^{-\beta u} n_{3} d u-n_{3}^{*}\right)}{-\left(1-S\left(n_{3}^{*}, n_{3}^{2}\right)-n_{3}^{*}\right) \int_{0}^{+\infty} e^{-\beta u}\left(\frac{Q_{\mathrm{n}}}{p_{\mathrm{n}}} S\left(n_{3}, n_{3}^{*}\right)+1\right) d u+\frac{1}{p_{\mathrm{n}}} \int_{0}^{+\infty} e^{-\beta u} d S\left(n_{3}, n_{3}^{*}\right)} \\
& =\frac{\left(1+\frac{Q_{\mathrm{n}}}{p_{\mathrm{n}}} S\left(n_{3}^{*}, n_{3}^{2}\right)\right) \frac{\beta}{p_{\mathrm{n}}} \int_{0}^{+\infty} e^{-\beta u} S\left(n_{3}, n_{3}^{*}\right) d u+\frac{1}{p_{\mathrm{n}}}\left(\beta \int_{0}^{+\infty} e^{-\beta u} n_{3} d u-n_{3}^{*}\right)}{-\left(1-S\left(n_{3}^{*}, n_{3}^{2}\right)-n_{3}^{*}\right) \int_{0}^{+\infty} e^{-\beta u}\left(\frac{Q_{\mathrm{n}}}{p_{\mathrm{n}}} S\left(n_{3}, n_{3}^{*}\right)+1\right) d u+\frac{\beta}{p_{\mathrm{n}}} \int_{0}^{+\infty} e^{-\beta u} S\left(n_{3}, n_{3}^{*}\right) d u} \\
& =\frac{\frac{\left(p_{\mathrm{n}}+q_{\mathrm{n}} n_{3}^{*}\right)^{\frac{Q_{\mathrm{n}}}{q_{\mathrm{n}}}}}{\left(p_{\mathrm{n}}+q_{\mathrm{n}} n_{3}^{2}\right)^{\frac{Q_{\mathrm{n}}}{q_{\mathrm{n}}}}}+\frac{\int_{0}^{+\infty} e^{-\beta u}\left(n_{3}-n_{3}^{*}\right) d u}{\int_{0}^{+\infty} e^{-\beta u} S\left(n_{3}, n_{3}^{*}\right) d u}}{1+\left(n_{3}^{*}+S\left(n_{3}^{*}, n_{3}^{2}\right)-1\right)\left(\frac{Q_{\mathrm{n}}}{\beta}+\frac{p_{\mathrm{n}}}{\beta^{2} \int_{0}^{+\infty} e^{-\beta u} S\left(n_{3}, n_{3}^{*}\right) d u}\right)}=\frac{\frac{1}{\left(p_{\mathrm{n}}+q_{\mathrm{n}} n_{3}^{2}\right)^{\frac{Q_{\mathrm{n}}}{q_{\mathrm{n}}}}+\frac{q_{\mathrm{n}}}{p_{\mathrm{n}}} A\left(n_{3}^{*}\right)}}{B\left(n_{3}^{*}\right)}
\end{aligned}
$$

with

$$
\begin{aligned}
& A\left(n_{3}^{*}\right)= \frac{\delta \int_{0}^{+\infty} e^{-\beta u}\left(n_{3}-n_{3}^{*}\right) d u}{\int_{0}^{+\infty} e^{-\beta u}\left(\left(p_{\mathrm{n}}+q_{\mathrm{n}} n_{3}\right)^{\delta}-\left(p_{\mathrm{n}}+q_{\mathrm{n}} n_{3}^{*}\right)^{\delta}\right) d u}, \\
& B\left(n_{3}^{*}\right)=\frac{1+\left(n_{3}^{*}+S\left(n_{3}^{*}, n_{3}^{2}\right)-1\right)\left(\frac{Q_{\mathrm{n}}}{\beta}+\frac{p_{\mathrm{n}}}{\beta^{2} \int_{0}^{+\infty} e^{-\beta u} S\left(n_{3}, n_{3}^{*}\right) d u}\right)}{\left(p_{\mathrm{n}}+q_{\mathrm{n}} n_{3}^{*}\right)^{\delta}},
\end{aligned}
$$

where we have used $\delta=\frac{Q_{\mathrm{n}}}{q_{\mathrm{n}}} \geq 1$.

Below we will show that under the assumptions of the proposition $A$ is a non-increasing, and $B$ is a non-decreasing function of $n_{3}^{*}$. For convenience, we state these results as Lemmata.

\section{Lemma A1}

For $\beta \leq 3 p_{\mathrm{n}}, A$ is a non-increasing function of $n_{3}^{*}$.

\section{Proof}

First,

$$
\begin{aligned}
A\left(n_{3}^{*}\right) & =\frac{\delta \int_{0}^{+\infty} e^{-\beta u}\left(n_{3}-n_{3}^{*}\right) d u}{\int_{0}^{+\infty} e^{-\beta u}\left(\left(p_{\mathrm{n}}+q_{\mathrm{n}} n_{3}\right)^{\delta}-\left(p_{\mathrm{n}}+q_{\mathrm{n}} n_{3}^{*}\right)^{\delta}\right) d u}=\frac{1}{q} \frac{\delta \int_{0}^{+\infty} e^{-\beta u}\left(\left(p_{\mathrm{n}}+q_{\mathrm{n}} n_{3}\right)-\left(p_{\mathrm{n}}+q_{\mathrm{n}} n_{3}^{*}\right) d u\right.}{\int_{0}^{+\infty} e^{-\beta u}\left(\left(p_{\mathrm{n}}+q_{\mathrm{n}} n_{3}\right)^{\delta}-\left(p_{\mathrm{n}}+q_{\mathrm{n}} n_{3}^{*}\right)^{\delta}\right) d u} \\
& =\frac{1}{q_{\mathrm{n}} p_{\mathrm{n}}^{\delta-1}} \frac{\delta \int_{0}^{+\infty} e^{-\beta u}\left((1+y)-\left(1+y^{*}\right) d u\right.}{\int_{0}^{+\infty} e^{-\beta u}\left((1+y)^{\delta}-\left(1+y^{*}\right)^{\delta}\right) d u}
\end{aligned}
$$


where $y=\frac{q_{\mathrm{n}} n_{3}}{p_{\mathrm{n}}}$ and $y^{*}=\frac{q_{\mathrm{n}} n_{3}^{*}}{p_{\mathrm{n}}}$. Note that the monotonicity of $A$ with respect to $n_{3}^{*}$ is equivalent to the monotonicity of

$$
D=\frac{\delta \int_{0}^{+\infty} e^{-\beta u}\left((1+y)-\left(1+y^{*}\right) d u\right.}{\int_{0}^{+\infty} e^{-\beta u}\left((1+y)^{\delta}-\left(1+y^{*}\right)^{\delta}\right) d u}
$$

with respect to $y^{*}$. Further, the sign of $\frac{d A}{d y^{*}}$ is the same as

$$
\begin{aligned}
& \delta\left(\int_{0}^{+\infty} e^{-\beta u}\left(\frac{d y}{d y^{*}}-1\right) d u\right)\left(\int_{0}^{+\infty} e^{-\beta u}\left((1+y)^{\delta}-\left(1+y^{*}\right)^{\delta}\right) d u\right) \\
& -\delta\left(\int_{0}^{+\infty} e^{-\beta u}\left((1+y)^{\delta-1} \frac{d y}{d y^{*}}-\left(1+y^{*}\right)^{\delta-1}\right) d u\right)\left(\int_{0}^{+\infty} e^{-\beta u}\left(y-y^{*}\right) d u\right) \\
\leq & \delta\left(\int_{0}^{+\infty} e^{-\beta u}\left(\frac{d y}{d y^{*}}-1\right) d u\right)\left(\int_{0}^{+\infty} e^{-\beta u}\left((1+y)^{\delta}-\left(1+y^{*}\right)^{\delta}\right) d u\right) \\
& -\delta\left(1+y^{*}\right)^{\delta-1}\left(\int_{0}^{+\infty} e^{-\beta u}\left(\frac{d y}{d y^{*}}-1\right) d u\right)\left(\int_{0}^{+\infty} e^{-\beta u}\left(y-y^{*}\right) d u\right) \\
= & \delta\left(\int_{0}^{+\infty} e^{-\beta u}\left(\frac{d y}{d y^{*}}-1\right) d u\right) \\
& \times\left(\int_{0}^{+\infty} e^{-\beta u}\left((1+y)^{\delta}-\left(1+y^{*}\right)^{\delta}\right) d u-\left(1+y^{*}\right)^{\delta-1} \int_{0}^{+\infty} e^{-\beta u}\left(y-y^{*}\right) d u\right) .
\end{aligned}
$$

Note that

$$
\begin{aligned}
& \left(\int_{0}^{+\infty} e^{-\beta u}\left((1+y)^{\delta}-\left(1+y^{*}\right)^{\delta}\right) d u-\left(1+y^{*}\right)^{\delta-1} \int_{0}^{+\infty} e^{-\beta u}\left(y-y^{*}\right) d u\right) \\
= & \left(1+y^{*}\right)^{\delta}\left(\int_{0}^{+\infty} e^{-\beta u}\left(\left(\frac{1+y}{1+y^{*}}\right)^{\delta}-\left(\frac{1+y}{1+y^{*}}\right)\right) d u\right) \\
= & \left(1+y^{*}\right)^{\delta}\left(\int_{0}^{+\infty} e^{-\beta u}\left(\frac{1+y}{1+y^{*}}\right)\left(\left(\frac{1+y}{1+y^{*}}\right)^{\delta-1}-1\right) d u\right) \geq 0
\end{aligned}
$$

for $y \geq y^{*}$ and $\delta \geq 1$. Since $\frac{d y}{d y^{*}}=\frac{d n_{3}}{d n_{3}^{*}}$ and

$$
\frac{d n_{3}}{d n_{3}^{*}}-1=\frac{\left(p_{\mathrm{n}}+q_{\mathrm{n}} n_{3}\right)\left(1-S\left(n_{3}^{*}, n_{3}^{2}\right)-n_{3}-S\left(n_{3}, n_{3}^{*}\right)\left(2+\frac{Q_{\mathrm{n}}}{p_{\mathrm{n}}} S\left(n_{3}^{*}, n_{3}^{2}\right)\right)\right)}{\left(p_{\mathrm{n}}+q_{\mathrm{n}} n_{3}^{*}\right)\left(1-S\left(n_{3}^{*}, n_{3}^{2}\right)-n_{3}^{*}\right)},
$$

so that

$$
\int_{0}^{+\infty} d u e^{-\beta u}\left(\frac{d y}{d y^{*}}-1\right)=\int_{0}^{+\infty} d u e^{-\beta u}\left(\frac{\frac{d n_{3}}{d u}-\left(p_{\mathrm{n}}+q_{\mathrm{n}} n_{3}^{*}\right)\left(1-S\left(n_{3}^{*}, n_{3}^{2}\right)-n_{3}^{*}\right)}{\left(p_{\mathrm{n}}+q_{\mathrm{n}} n_{3}^{*}\right)\left(1-S\left(n_{3}^{*}, n_{3}^{2}\right)-n_{3}^{*}\right)}\right) .
$$


Now,

$$
\begin{aligned}
& \int_{0}^{+\infty} d u e^{-\beta u}\left(\frac{d n_{3}}{d u}-\left(p_{\mathrm{n}}+q_{\mathrm{n}} n_{3}^{*}\right)\left(1-S\left(n_{3}^{*}, n_{3}^{2}\right)-n_{3}^{*}\right)\right) \\
= & -n_{3}^{*}+\beta \int_{0}^{+\infty} n_{3} d u e^{-\beta u}-\frac{1}{\beta}\left(p_{\mathrm{n}}+q_{\mathrm{n}} n_{3}^{*}\right)\left(1-S\left(n_{3}^{*}, n_{3}^{2}\right)-n_{3}^{*}\right) \\
\leq & \bar{n}_{3}^{*}-n_{3}^{*}-\frac{1}{\beta}\left(p_{\mathrm{n}}+q_{\mathrm{n}} n_{3}^{*}\right)\left(1-S\left(n_{3}^{*}, n_{3}^{2}\right)-n_{3}^{*}\right) .
\end{aligned}
$$

where $\bar{n}_{3}^{*}=\lim _{u \rightarrow+\infty}\left(n_{3}\right)$ is the limiting customer base indicator of the new entrant firm, for given values of $n_{3}^{2}$ and $n_{3}^{*}$. According to (47), $\bar{n}_{3}^{*}$ is the solution to the following equation

$$
1-S\left(n_{3}^{*}, n_{3}^{2}\right)-\bar{n}_{3}^{*}-S\left(\bar{n}_{3}^{*}, n_{3}^{*}\right)\left(2+\frac{Q_{\mathrm{n}}}{p_{\mathrm{n}}} S\left(n_{3}^{*}, n_{3}^{2}\right)\right)=0 .
$$

Note that the left hand-side of (A153) is non-positive for any $\beta \leq \beta^{*}$, where

$$
\begin{aligned}
\beta^{*} & =\inf _{n_{3}^{2}, n_{3}^{*} \geq n_{3}^{2}}\left(\frac{\left(p_{\mathrm{n}}+q_{\mathrm{n}} n_{3}^{*}\right)\left(1-S\left(n_{3}^{*}, n_{3}^{2}\right)-n_{3}^{*}\right)}{\bar{n}_{3}^{*}-n_{3}^{*}}\right) \\
& =\inf _{n_{3}^{2}, n_{3}^{*} \geq n_{3}^{2}}\left(p_{\mathrm{n}}+q_{\mathrm{n}} n_{3}^{*}+\left(p_{\mathrm{n}}+q_{\mathrm{n}} n_{3}^{*}\right) \frac{S\left(\bar{n}_{3}^{*}, n_{3}^{*}\right)\left(2+\frac{Q_{\mathrm{n}}}{p_{\mathrm{n}}} S\left(n_{3}^{*}, n_{3}^{2}\right)\right)}{\bar{n}_{3}^{*}-n_{3}^{*}}\right) .
\end{aligned}
$$

Now, consider the expression

$$
\begin{aligned}
G & =\left(p_{\mathrm{n}}+q_{\mathrm{n}} n_{3}^{*}\right) \frac{S\left(\bar{n}_{3}^{*}, n_{3}^{*}\right)}{\bar{n}_{3}^{*}-n_{3}^{*}}=\frac{q_{\mathrm{n}}\left(p_{\mathrm{n}}+q_{\mathrm{n}} n_{3}^{*}\right)}{\left(p_{\mathrm{n}}+q_{\mathrm{n}} \bar{n}_{3}^{*}\right)-\left(p_{\mathrm{n}}+q_{\mathrm{n}} n_{3}^{*}\right)}\left(\frac{p_{\mathrm{n}}}{Q_{\mathrm{n}}}\left(\left(\frac{p_{\mathrm{n}}+q_{\mathrm{n}} \bar{n}_{3}^{*}}{p_{\mathrm{n}}+q_{\mathrm{n}} n_{3}^{*}}\right)^{\delta}-1\right)\right) \\
& =\frac{p_{\mathrm{n}}}{\delta} \frac{\left(\frac{p_{\mathrm{n}}+q_{\mathrm{n}} \bar{n}_{3}^{*}}{p_{\mathrm{n}}+q_{\mathrm{n}} n_{3}^{*}}\right)^{\delta}-1}{\left(\frac{p_{\mathrm{n}}+q_{\mathrm{n}} \bar{n}_{3}^{*}}{p_{\mathrm{n}}+q_{\mathrm{n}} n_{3}^{*}}\right)-1}=\frac{p_{\mathrm{n}}}{\delta}\left(\frac{(1+F)^{\delta}-1}{F}\right),
\end{aligned}
$$

where

$$
F=\left(\frac{p_{\mathrm{n}}+q_{\mathrm{n}} \bar{n}_{3}^{*}}{p_{\mathrm{n}}+q_{\mathrm{n}} n_{3}^{*}}\right)-1 \geq 0 .
$$

Note that

$$
\begin{aligned}
\frac{d G}{d n_{3}^{*}} & =\frac{p_{\mathrm{n}}}{\delta}\left(\frac{\delta(1+F)^{\delta-1} F-\left((1+F)^{\delta}-1\right)}{F^{2}}\right) \frac{d F}{d n_{3}^{*}} \\
& =\frac{p_{\mathrm{n}}}{\delta}\left(\frac{1+(\delta-1)(1+F)^{\delta-1} F-(1+F)^{\delta-1}}{F^{2}}\right) \frac{d F}{d n_{3}^{*}} \\
& =\frac{p_{\mathrm{n}}}{\delta}\left(\frac{(1+F)^{\delta-1}\left(\frac{1}{(1+F)^{\delta-1}}+(\delta-1) F-1\right)}{F^{2}}\right) \frac{d F}{d n_{3}^{*}} .
\end{aligned}
$$


Since $\frac{1}{(1+F)^{\delta-1}}+(\delta-1) F-1$ is an increasing function of $F$ for $F \geq 0$, the smallest value for this expression is 0 (reached at $F=0$ ). Thus, the sign of $\frac{d G}{d n_{3}^{*}}$ is the same as the sign of $\frac{d F}{d n_{3}^{*}}$. Now,

$$
\frac{d F}{d n_{3}^{*}}=\frac{q \frac{d \bar{n}_{3}^{*}}{d n_{3}^{*}}\left(p_{\mathrm{n}}+q_{\mathrm{n}} n_{3}^{*}\right)-q_{\mathrm{n}}\left(p_{\mathrm{n}}+q_{\mathrm{n}} \bar{n}_{3}^{*}\right)}{\left(p_{\mathrm{n}}+q_{\mathrm{n}} n_{3}^{*}\right)^{2}}=\frac{q_{\mathrm{n}}\left(p_{\mathrm{n}}+q_{\mathrm{n}} \bar{n}_{3}^{*}\right)}{\left(p_{\mathrm{n}}+q_{\mathrm{n}} n_{3}^{*}\right)^{2}}\left(\frac{\left(p_{\mathrm{n}}+q_{\mathrm{n}} n_{3}^{*}\right) d \bar{n}_{3}^{*}}{\left(p_{\mathrm{n}}+q_{\mathrm{n}} \bar{n}_{3}^{*}\right) d n_{3}^{*}}-1\right) .
$$

Using (A154), we get

$$
\begin{aligned}
0= & -\frac{p_{\mathrm{n}}}{p_{\mathrm{n}}+q_{\mathrm{n}} n_{3}^{*}}\left(1+\frac{Q_{\mathrm{n}}}{p_{\mathrm{n}}} S\left(n_{3}^{*}, n_{3}^{2}\right)\right)-\frac{d \bar{n}_{3}^{*}}{d n_{3}^{*}} \\
& -\left(\frac{p_{\mathrm{n}}}{p_{\mathrm{n}}+q_{\mathrm{n}} \bar{n}_{3}^{*}} \frac{d \bar{n}_{3}^{*}}{d n_{3}^{*}}-\frac{p_{\mathrm{n}}}{p_{\mathrm{n}}+q_{\mathrm{n}} n_{3}^{*}}\right)\left(1+\frac{Q_{\mathrm{n}}}{p_{\mathrm{n}}} S\left(\bar{n}_{3}^{*}, n_{3}^{*}\right)\right)\left(2+\frac{Q_{\mathrm{n}}}{p_{\mathrm{n}}} S\left(n_{3}^{*}, n_{3}^{2}\right)\right) \\
& -\frac{Q_{\mathrm{n}}}{p_{\mathrm{n}}} S\left(\bar{n}_{3}^{*}, n_{3}^{*}\right) \frac{p_{\mathrm{n}}}{p_{\mathrm{n}}+q_{\mathrm{n}} n_{3}^{*}}\left(1+\frac{Q_{\mathrm{n}}}{p_{\mathrm{n}}} S\left(n_{3}^{*}, n_{3}^{2}\right)\right),
\end{aligned}
$$

or

$$
\frac{\left(p_{\mathrm{n}}+q_{\mathrm{n}} n_{3}^{*}\right) d \bar{n}_{3}^{*}}{\left(p_{\mathrm{n}}+q_{\mathrm{n}} \bar{n}_{3}^{*}\right) d n_{3}^{*}}-1=\frac{-\left(p_{\mathrm{n}}+q_{\mathrm{n}} \bar{n}_{3}^{*}\right)-p_{\mathrm{n}}\left(1+\frac{Q_{\mathrm{n}}}{p_{\mathrm{n}}} S\left(\bar{n}_{3}^{*}, n_{3}^{*}\right)\right)\left(1+\frac{Q_{\mathrm{n}}}{p_{\mathrm{n}}} S\left(n_{3}^{*}, n_{3}^{2}\right)\right)}{p_{\mathrm{n}}+q_{\mathrm{n}} \bar{n}_{3}^{*}+p_{\mathrm{n}}\left(1+\frac{Q_{\mathrm{n}}}{p_{\mathrm{n}}} S\left(\bar{n}_{3}^{*}, n_{3}^{*}\right)\right)\left(2+\frac{Q_{\mathrm{n}}}{p_{\mathrm{n}}} S\left(n_{3}^{*}, n_{3}^{2}\right)\right)} \leq 0,
$$

so that $\frac{d G}{d n_{3}^{*}} \leq 0$. Then,

$$
\begin{aligned}
\beta^{*} & =\inf _{n_{3}^{2}, n_{3}^{*} \geq n_{3}^{2}}\left(p_{\mathrm{n}}+q_{\mathrm{n}} n_{3}^{*}+G\left(2+\frac{Q_{\mathrm{n}}}{p_{\mathrm{n}}} S\left(n_{3}^{*}, n_{3}^{2}\right)\right)\right) \\
& \geq \inf _{n_{3}^{2}, n_{3}^{*} \geq n_{3}^{2}}\left(p_{\mathrm{n}}+q_{\mathrm{n}} n_{3}^{*}\right)+\inf _{n_{3}^{2}, n_{3}^{*} \geq n_{3}^{2}}\left(G\left(2+\frac{Q_{\mathrm{n}}}{p_{\mathrm{n}}} S\left(n_{3}^{*}, n_{3}^{2}\right)\right)\right) \\
& \geq p_{\mathrm{n}}+p_{\mathrm{n}} \inf _{n_{3}^{2}, n_{3}^{*} \geq n_{3}^{2}}\left(2+\frac{Q_{\mathrm{n}}}{p_{\mathrm{n}}} S\left(n_{3}^{*}, n_{3}^{2}\right)\right)=3 p_{\mathrm{n}},
\end{aligned}
$$

where we have used monotonicity of $G$ and $S$ functions. Thus, any $\beta \leq 3 p_{\text {n }}$ also satisfies $\beta \leq \beta^{*}$. Thus, $A$ is a non-increasing function of $n_{3}^{*}$ for $\beta \leq 3 p_{\mathrm{n}}$.

\section{Lemma A2}

For $\beta \leq 3 p_{\mathrm{n}}, B$ is a non-decreasing function of $n_{3}^{*}$.

\section{Proof}

From (A146),

$$
\begin{aligned}
& B\left(n_{3}^{*}\right)=\frac{1+\left(n_{3}^{*}+S\left(n_{3}^{*}, n_{3}^{2}\right)-1\right)\left(\frac{Q_{\mathrm{n}}}{\beta}+\frac{p_{\mathrm{n}}}{\beta^{2} \int_{0}^{+\infty} e^{-\beta u} S\left(n_{3}, n_{3}^{*}\right) d u}\right)}{\left(p_{\mathrm{n}}+q_{\mathrm{n}} n_{3}^{*}\right)^{\delta}} \\
& =\frac{1}{\left(p_{\mathrm{n}}+q_{\mathrm{n}} n_{3}^{*}\right)^{\delta}}+\frac{Q_{\mathrm{n}}}{\beta} \frac{\left(n_{3}^{*}+S\left(n_{3}^{*}, n_{3}^{2}\right)-1\right)}{\left(p_{\mathrm{n}}+q_{\mathrm{n}} n_{3}^{*}\right)^{\delta}}+\frac{p_{\mathrm{n}}\left(n_{3}^{*}+S\left(n_{3}^{*}, n_{3}^{2}\right)-1\right)}{\beta^{2}\left(p_{\mathrm{n}}+q_{\mathrm{n}} n_{3}^{*}\right)^{\delta} \int_{0}^{+\infty} e^{-\beta u} S\left(n_{3}, n_{3}^{*}\right) d u} .
\end{aligned}
$$


Now,

$$
\begin{aligned}
\frac{d}{d n_{3}^{*}}\left(\frac{n_{3}^{*}+S\left(n_{3}^{*}, n_{3}^{2}\right)-1}{\left(p_{\mathrm{n}}+q_{\mathrm{n}} n_{3}^{*}\right)^{\delta}}\right) & =\frac{d}{d n_{3}^{*}}\left(\frac{n_{3}^{*}-\frac{p_{\mathrm{n}}}{Q_{\mathrm{n}}}-1}{\left(p_{\mathrm{n}}+q_{\mathrm{n}} n_{3}^{*}\right)^{\delta}}\right)=\frac{\left(p_{\mathrm{n}}+q_{\mathrm{n}} n_{3}^{*}\right)^{\delta}-\delta q_{\mathrm{n}}\left(p_{\mathrm{n}}+q_{\mathrm{n}} n_{3}^{*}\right)^{\delta-1}\left(n_{3}^{*}-\frac{p_{\mathrm{n}}}{Q_{\mathrm{n}}}-1\right)}{\left(p_{\mathrm{n}}+q_{\mathrm{n}} n_{3}^{*}\right)^{2 \delta}} \\
& =\frac{1}{\left(p_{\mathrm{n}}+q_{\mathrm{n}} n_{3}^{*}\right)^{\delta+1}}\left(q_{\mathrm{n}} n_{3}^{*}(1-\delta)+2 p_{\mathrm{n}}+\delta q\right),
\end{aligned}
$$

and

$$
\begin{aligned}
& \frac{d}{d n_{3}^{*}}\left(\frac{p_{\mathrm{n}}\left(n_{3}^{*}+S\left(n_{3}^{*}, n_{3}^{2}\right)-1\right)}{\beta^{2}\left(p_{\mathrm{n}}+q_{\mathrm{n}} n_{3}^{*}\right)^{\delta} \int_{0}^{+\infty} e^{-\beta u} S\left(n_{3}, n_{3}^{*}\right) d u}\right)=\frac{p_{\mathrm{n}}}{\beta^{2} \int_{0}^{+\infty} e^{-\beta u} S\left(n_{3}, n_{3}^{*}\right) d u} \frac{d}{d n_{3}^{*}}\left(\frac{n_{3}^{*}+S\left(n_{3}^{*}, n_{3}^{2}\right)-1}{\left(p_{\mathrm{n}}+q_{\mathrm{n}} n_{3}^{*}\right)^{\delta}}\right) \\
& +\frac{p_{\mathrm{n}}}{\beta^{2}}\left(\frac{n_{3}^{*}+S\left(n_{3}^{*}, n_{3}^{2}\right)-1}{\left(p_{\mathrm{n}}+q_{\mathrm{n}} n_{3}^{*}\right)^{\delta}}\right) \frac{d}{d n_{3}^{*}}\left(\frac{1}{\int_{0}^{+\infty} e^{-\beta u} S\left(n_{3}, n_{3}^{*}\right) d u}\right)
\end{aligned}
$$

Further,

$$
\begin{aligned}
& \frac{d}{d n_{3}^{*}}\left(\frac{1}{\int_{0}^{+\infty} e^{-\beta u} S\left(n_{3}, n_{3}^{*}\right) d u}\right)=-\frac{\frac{d}{d n_{3}^{*}}\left(\int_{0}^{+\infty} e^{-\beta u} S\left(n_{3}, n_{3}^{*}\right) d u\right)}{\left(\int_{0}^{+\infty} e^{-\beta u} S\left(n_{3}, n_{3}^{*}\right) d u\right)^{2}} \\
= & \frac{\int_{0}^{+\infty} e^{-\beta u}\left(\left(\frac{p_{\mathrm{n}}}{p_{\mathrm{n}}+q_{\mathrm{n}} n_{3}^{*}}\right)-\left(\frac{p_{\mathrm{n}}}{p_{\mathrm{n}}+q_{\mathrm{n}} n_{3}}\right) \frac{d n_{3}}{d n_{3}^{*}}\right)\left(1+\frac{Q_{\mathrm{n}}}{p_{\mathrm{n}}} S\left(n_{3}, n_{3}^{*}\right)\right) d u}{\left(\int_{0}^{+\infty} e^{-\beta u} S\left(n_{3}, n_{3}^{*}\right) d u\right)^{2}} .
\end{aligned}
$$

As (47) indicates,

$$
\frac{d n_{3}(t)}{d n_{3}\left(t^{*}\right)}=\frac{\left(p_{\mathrm{n}}+q_{\mathrm{n}} n_{3}(t)\right)\left(1-S\left(n_{3}\left(t^{*}\right), n_{3}\left(t_{2}\right)\right)-n_{3}(t)-S\left(n_{3}(t), n_{3}\left(t^{*}\right)\right)\left(2+\frac{Q_{\mathrm{n}}}{p_{\mathrm{n}}} S\left(n_{3}\left(t^{*}\right), n_{3}\left(t_{2}\right)\right)\right)\right)}{\left(p_{\mathrm{n}}+q_{\mathrm{n}} n_{3}\left(t^{*}\right)\right)\left(1-S\left(n_{3}\left(t^{*}\right), n_{3}\left(t_{2}\right)\right)-n_{3}\left(t^{*}\right)\right)} .
$$

Using (A167), we get

$$
\begin{aligned}
& \int_{0}^{+\infty} e^{-\beta u}\left(\left(\frac{p_{\mathrm{n}}}{p_{\mathrm{n}}+q_{\mathrm{n}} n_{3}}\right) \frac{d n_{3}}{d n_{3}^{*}}-\left(\frac{p_{\mathrm{n}}}{p_{\mathrm{n}}+q_{\mathrm{n}} n_{3}^{*}}\right)\right)\left(1+\frac{Q_{\mathrm{n}}}{p_{\mathrm{n}}} S\left(n_{3}, n_{3}^{*}\right)\right) d u \\
= & \left(\frac{p_{\mathrm{n}}}{p_{\mathrm{n}}+q_{\mathrm{n}} n_{3}^{*}}\right) \\
& \times \int_{0}^{+\infty} e^{-\beta u} d u\left(\frac{n_{3}^{*}-n_{3}-S\left(n_{3}, n_{3}^{*}\right)\left(2+\frac{Q_{\mathrm{n}}}{p_{\mathrm{n}}} S\left(n_{3}^{*}, n_{3}^{2}\right)\right)}{\left(1-S\left(n_{3}^{*}, n_{3}^{2}\right)-n_{3}^{*}\right)}\right)\left(1+\frac{Q_{\mathrm{n}}}{p_{\mathrm{n}}} S\left(n_{3}, n_{3}^{*}\right)\right),
\end{aligned}
$$


and

$$
\begin{aligned}
& \frac{d}{d n_{3}^{*}}\left(\frac{1}{\int_{0}^{+\infty} e^{-\beta u} S\left(n_{3}, n_{3}^{*}\right) d u}\right)=\left(\frac{p_{\mathrm{n}}}{p_{\mathrm{n}}+q_{\mathrm{n}} n_{3}^{*}}\right) \\
& \times \frac{\int_{0}^{+\infty} e^{-\beta u}\left(\frac{n_{3}(t)-n_{3}^{*}+S\left(n_{3}(t), n_{3}^{*}\right)\left(2+\frac{Q_{\mathrm{n}}}{p_{\mathrm{n}}} S\left(n_{3}^{*}, n_{3}^{2}\right)\right)}{\left(1-S\left(n_{3}^{*}, n_{3}^{2}\right)-n_{3}^{*}\right)}\right)\left(1+\frac{Q_{\mathrm{n}}}{p_{\mathrm{n}}} S\left(n_{3}, n_{3}^{*}\right)\right) d u}{\left(\int_{0}^{+\infty} e^{-\beta u} S\left(n_{3}, n_{3}^{*}\right) d u\right)^{2}} \\
& \geq 0 \text {. }
\end{aligned}
$$

Putting these results together, we get

$$
\begin{aligned}
\frac{d B\left(n_{3}^{*}\right)}{d n_{3}^{*}}= & -\frac{\delta q_{\mathrm{n}}}{\left(p_{\mathrm{n}}+q_{\mathrm{n}} n_{3}^{*}\right)^{\delta+1}}+\left(\frac{Q_{\mathrm{n}}}{\beta}+\frac{p_{\mathrm{n}}}{\beta^{2} \int_{0}^{+\infty} e^{-\beta u} S\left(n_{3}, n_{3}^{*}\right) d u}\right) \frac{\left(q_{\mathrm{n}} n_{3}^{*}(1-\delta)+2 p_{\mathrm{n}}+\delta q_{\mathrm{n}}\right)}{\left(p_{\mathrm{n}}+q_{\mathrm{n}} n_{3}^{*}\right)^{\delta+1}} \\
& +\frac{p_{\mathrm{n}}}{\beta^{2}}\left(\frac{n_{3}^{*}+S\left(n_{3}^{*}, n_{3}^{2}\right)-1}{\left(p_{\mathrm{n}}+q_{\mathrm{n}} n_{3}^{*}\right)^{\delta}}\right) \frac{d}{d n_{3}^{*}}\left(\frac{1}{\int_{0}^{+\infty} e^{-\beta u} S\left(n_{3}, n_{3}^{*}\right) d u}\right) \\
\geq & -\frac{\delta q_{\mathrm{n}}}{\left(p_{\mathrm{n}}+q_{\mathrm{n}} n_{3}^{*}\right)^{\delta+1}}+\left(\frac{Q_{\mathrm{n}}}{\beta}+\frac{\left(q n_{3}^{*}(1-\delta)+2 p_{\mathrm{n}}+\delta q_{\mathrm{n}}\right)}{\left(p_{\mathrm{n}}+q_{\mathrm{n}} n_{3}^{*}\right)^{\delta+1}}\right. \\
\geq & \left.-\frac{\delta q_{\mathrm{n}}}{\left(p_{\mathrm{n}}+q_{\mathrm{n}} n_{3}^{*}\right)^{\delta+1}}+\left(\frac{Q_{\mathrm{n}}}{\beta}+\frac{p_{\mathrm{n}}}{\beta}\right) \frac{\left(q_{\mathrm{n}} n_{3}^{*}(1-\delta)+2 n_{3}, n_{3}^{*}\right) d u}{\left(p_{\mathrm{n}}+q_{\mathrm{n}} n_{3}^{*}\right)^{\delta+1}}\right) \\
\geq & -\frac{Q_{\mathrm{n}}}{\left(p_{\mathrm{n}}+q_{\mathrm{n}} n_{3}^{*}\right)^{\delta+1}}+\left(\frac{Q_{\mathrm{n}}}{\beta}+\frac{p_{\mathrm{n}}}{\beta}\right) \frac{\left(q_{\mathrm{n}}+2 p_{\mathrm{n}}\right)}{\left(p_{\mathrm{n}}+q_{\mathrm{n}} n_{3}^{*}\right)^{\delta+1}} \geq 0
\end{aligned}
$$

for any $\beta \leq \frac{\left(Q_{\mathrm{n}}+p_{\mathrm{n}}\right)\left(q_{\mathrm{n}}+2 p_{\mathrm{n}}\right)}{Q_{\mathrm{n}}}$, in particular, for $\beta \leq 3 p_{\mathrm{n}}$. Note that in deriving (A170) we have used (A169), as well as $S\left(n_{3}, n_{3}^{*}\right) \leq 1$ and $n_{3}^{*} \leq 1$.

Thus, the second derivative of the profit function at any local optimum is non-positive - the profit function is quasiconcave.

\section{Proof of Proposition 10}

Let $\rho=\frac{\pi_{\mathrm{o}} \theta}{\pi_{\mathrm{n}}}$ and define $T_{m}$ as a set of local unconstrained maxima of $\Pi_{1}\left(t_{1}, t_{2}\right)$ :

$$
T_{m}=\left(0<t<t_{\max }\left|\frac{\partial \Pi_{1}\left(t_{1}, t_{2}\right)}{\partial t_{1}}\right|_{t_{1}=t}=0,\left.\frac{\partial^{2} \Pi_{1}\left(t_{1}, t_{2}\right)}{\partial\left(t_{1}\right)^{2}}\right|_{t_{1}=t} \leq 0\right) .
$$


The statement of the Proposition will be established if we show that

$$
\frac{\partial t}{\partial \rho} \geq 0
$$

for any $t \in T_{m}$. Consider an arbitrary $t^{*} \in T_{m}$. Then,

$$
\left.\frac{\partial \Pi_{1}\left(t_{1}, t_{2}\right)}{\partial t_{1}}\right|_{t_{1}=t^{*}}=0
$$

and

$$
\left.\frac{\partial^{2} \Pi_{1}\left(t_{1}, t_{2}\right)}{\partial t_{1} \partial \rho}\right|_{t_{1}=t^{*}}+\left.\frac{\partial t^{*}}{\partial \rho} \frac{\partial^{2} \Pi_{1}\left(t_{1}, t_{2}\right)}{\partial\left(t_{1}\right)^{2}}\right|_{t_{1}=t^{*}}=0 .
$$

Consider $t^{*} \geq t_{2}$ (the proof for the case of $t^{*} \leq t_{2}$ follows similar steps). Then,

$$
\left.\frac{\partial \Pi_{1}\left(t_{1}, t_{2}\right)}{\partial t_{1}}\right|_{t_{1}=t^{*}}=0 \Leftrightarrow \rho\left(\int_{t^{*}}^{+\infty} e^{-\beta t}\left(\frac{\partial o_{13}\left(t, t^{*}, t_{2}\right)}{\partial t^{*}}\right) d t\right)+\left(\int_{t^{*}}^{+\infty} e^{-\beta t}\left(\frac{\partial n_{1}\left(t, t^{*}, t_{2}\right)}{\partial t^{*}}\right) d t\right)=0,
$$

and

$$
\begin{aligned}
\left.\frac{\partial^{2} \Pi_{1}\left(t_{1}, t_{2}\right)}{\partial\left(t_{1}\right)^{2}}\right|_{t_{1}=t^{*}} \leq & 0 \Leftrightarrow \rho\left(\int_{t^{*}}^{+\infty} e^{-\beta t}\left(\frac{\partial^{2} o_{13}\left(t, t^{*}, t_{2}\right)}{\partial\left(t^{*}\right)^{2}}\right) d t-e^{-\beta t^{*}}\left(\left.\frac{\partial o_{13}\left(t, t^{*}, t_{2}\right)}{\partial t^{*}}\right|_{t=t^{*}}\right)\right) \\
& +\left(\int_{t^{*}}^{+\infty} e^{-\beta t}\left(\frac{\partial^{2} n_{1}\left(t, t^{*}, t_{2}\right)}{\partial\left(t^{*}\right)^{2}}\right) d t-e^{-\beta t^{*}}\left(\left.\frac{\partial n_{1}\left(t, t^{*}, t_{2}\right)}{\partial t^{*}}\right|_{t=t^{*}}\right)\right) \\
\leq & 0,
\end{aligned}
$$

Differentiating (A174) with respect to $\rho$, we get

$$
\left(\int_{t^{*}}^{+\infty} e^{-\beta t}\left(\frac{\partial o_{13}\left(t, t^{*}, t_{2}\right)}{\partial t^{*}}\right) d t\right)+\frac{\partial t^{*}}{\partial \rho}\left(\left.\frac{\partial^{2} \Pi_{1}\left(t_{1}, t_{2}\right)}{\partial\left(t_{1}\right)^{2}}\right|_{t_{1}=t^{*}}\right)=0 .
$$

Note that, as (A174) indicates,

$$
\left(\int_{t^{*}}^{+\infty} e^{-\beta t}\left(\frac{\partial o_{13}\left(t, t^{*}, t_{2}\right)}{\partial t^{*}}\right) d t\right)=-\frac{1}{\rho}\left(\int_{t^{*}}^{+\infty} e^{-\beta t}\left(\frac{\partial n_{1}\left(t, t^{*}, t_{2}\right)}{\partial t^{*}}\right) d t\right) .
$$

Now, noting that $n_{3}(t)$ for $t \geq t^{*}$ is actually a function of $u=t-t^{*}$ and a parameter $n_{3}\left(t^{*}\right)$, $n_{3}(t)=f\left(u, n_{3}\left(t^{*}\right)\right)$, we can re-express the integral on the right-hand side of (A174) as

$$
\int_{t^{*}}^{+\infty} e^{-\beta t}\left(\frac{\partial n_{1}\left(t, t^{*}, t_{2}\right)}{\partial t^{*}}\right) d t
$$




$$
\begin{aligned}
& =e^{-\beta t^{*}} \int_{0}^{+\infty} e^{-\beta u}\left(\frac{\partial n_{1}}{\partial u}\right) d u \\
& =e^{-\beta t^{*}} \frac{d n_{3}\left(t^{*}\right)}{d t^{*}} \int_{0}^{+\infty} e^{-\beta u}\left(\frac{\partial S\left(n_{3}(t), n_{3}\left(t^{*}\right)\right)}{\partial n_{3}\left(t^{*}\right)}+\frac{\partial S\left(n_{3}(t), n_{3}\left(t^{*}\right)\right)}{\partial n_{3}(t)} \frac{d n_{3}(t)}{d n_{3}\left(t^{*}\right)}\right) d u
\end{aligned}
$$

where we have dropped the notation for a fixed value of $t_{2}$. Note that the second term in brackets in (A178) contains the limit of the ratio of the change of the function $S$ at any point $t$ to the change in the function value $n_{3}(t)$. Strictly speaking, we need to use the variance notation $\frac{\delta S\left(n_{3}(t), n_{3}\left(t^{*}\right)\right)}{\delta n_{3}(t)}$ here to denote such limit - however, we retain the "partial derivative" notation for simplicity. Using (28), we get

$$
\frac{\partial S\left(n_{3}(t), n_{3}\left(t^{*}\right)\right)}{\partial n_{3}\left(t^{*}\right)}=-\frac{p_{\mathrm{n}}}{p_{\mathrm{n}}+q_{\mathrm{n}} n_{3}\left(t^{*}\right)}\left(\frac{Q_{\mathrm{n}}}{p_{\mathrm{n}}} S\left(n_{3}(t), n_{3}\left(t^{*}\right)\right)+1\right),
$$

and

$$
\frac{\partial S\left(n_{3}(t), n_{3}\left(t^{*}\right)\right)}{\partial n_{3}(t)}=\frac{p_{\mathrm{n}}}{p_{\mathrm{n}}+q_{\mathrm{n}} n_{3}(t)}\left(\frac{Q_{\mathrm{n}}}{p_{\mathrm{n}}} S\left(n_{3}(t), n_{3}\left(t^{*}\right)\right)+1\right)
$$

Recall that from (A167),

$$
\frac{d n_{3}(t)}{d n_{3}\left(t^{*}\right)}=\frac{\left(p_{\mathrm{n}}+q_{\mathrm{n}} n_{3}(t)\right)\left(1-S\left(n_{3}\left(t^{*}\right), n_{3}\left(t_{2}\right)\right)-n_{3}(t)-S\left(n_{3}(t), n_{3}\left(t^{*}\right)\right)\left(2+\frac{Q_{\mathrm{n}}}{p_{\mathrm{n}}} S\left(n_{3}\left(t^{*}\right), n_{3}\left(t_{2}\right)\right)\right)\right)}{\left(p_{\mathrm{n}}+q_{\mathrm{n}} n_{3}\left(t^{*}\right)\right)\left(1-S\left(n_{3}\left(t^{*}\right), n_{3}\left(t_{2}\right)\right)-n_{3}\left(t^{*}\right)\right)} .
$$

Substituting (A179)-(A181) into (A178), we get

$$
\begin{aligned}
& \int_{t^{*}}^{+\infty} e^{-\beta t}\left(\frac{\partial n_{1}\left(t, t^{*}, t_{2}\right)}{\partial t^{*}}\right) d t \\
= & e^{-\beta t^{*}} \frac{d n_{3}\left(t^{*}\right)}{d t^{*}} \frac{p_{\mathrm{n}}}{p_{\mathrm{n}}+q_{\mathrm{n}} n_{3}\left(t^{*}\right)} \int_{0}^{+\infty} e^{-\beta u} d u\left(\frac{Q_{\mathrm{n}}}{p_{\mathrm{n}}} S\left(f\left(u, n_{3}\left(t^{*}\right)\right), n_{3}\left(t^{*}\right)\right)+1\right) \\
& \times\left(\frac{n_{3}\left(t^{*}\right)-f\left(u, n_{3}\left(t^{*}\right)\right)-S\left(f\left(u, n_{3}\left(t^{*}\right)\right), n_{3}\left(t^{*}\right)\right)\left(2+\frac{Q_{\mathrm{n}}}{p_{\mathrm{n}}} S\left(n_{3}\left(t^{*}\right), n_{3}\left(t_{2}\right)\right)\right)}{1-S\left(n_{3}\left(t^{*}\right), n_{3}\left(t_{2}\right)\right)-n_{3}\left(t^{*}\right)}\right) .
\end{aligned}
$$

Now, since $\frac{\partial n_{3}\left(t^{*}\right)}{\partial t^{*}}>0,1-S\left(n_{3}\left(t^{*}\right), n_{3}\left(t_{2}\right)\right)-n_{3}\left(t^{*}\right)>0$ (follows from $\left.(47)\right)$, and $n_{3}\left(t^{*}\right)-$ $f\left(u, n_{3}\left(t^{*}\right)\right)-S\left(f\left(u, n_{3}\left(t^{*}\right)\right), n_{3}\left(t^{*}\right)\right)\left(2+\frac{Q_{\mathrm{n}}}{p_{\mathrm{n}}} S\left(n_{3}\left(t^{*}\right), n_{3}\left(t_{2}\right)\right)\right) \leq 0$ for any $u \geq 0$ (equality is true only at $u=0$ ), integrals in (A182) are negative. Thus, the left-hand side of (A177) is positive. Combining this result with (A175) and (A176), we establish that $\frac{\partial t^{*}}{\partial \rho} \geq 0$. 


\section{Proof of Proposition 11}

a) Without loss of generality, consider the setting in which $t_{1} \geq t_{2}$. Then,

$$
\widehat{\Pi}_{1}\left(t_{1}, t_{2}\right)=\frac{\Pi_{1}\left(t_{1}, t_{2}\right)}{\pi_{n}}=\rho \bar{\Pi}_{\mathrm{o}}\left(t_{1}, t_{2}\right)+\bar{\Pi}_{\mathrm{n}}\left(t_{1}, t_{2}\right),
$$

where $\rho=\frac{\theta \pi_{\mathrm{o}}}{\pi_{\mathrm{n}}}$, and

$\bar{\Pi}_{\mathrm{o}}\left(t_{1}, t_{2}\right)=\int_{0}^{t_{2}} e^{-\beta t} o_{11}(t) d t+\int_{t_{2}}^{t_{1}} e^{-\beta t} o_{12}(t) d t+\int_{t_{1}}^{+\infty} e^{-\beta t} o_{13}(t) d t, \bar{\Pi}_{\mathrm{n}}\left(t_{1}, t_{2}\right)=\int_{t_{1}}^{+\infty} e^{-\beta t} S\left(n_{3}(t), n_{3}\left(t_{1}\right)\right) d t$.

Similarly, for firm 2 we get, after some algebra,

$$
\begin{aligned}
\widehat{\Pi}_{2}\left(t_{1}, t_{2}\right)= & \frac{\Pi_{2}\left(t_{1}, t_{2}\right)}{\pi_{n}}=\bar{\rho} \bar{\Pi}_{\mathrm{o}}\left(t_{1}, t_{2}\right)+\bar{\Pi}_{\mathrm{n}}\left(t_{1}, t_{2}\right)\left(1+\frac{Q_{\mathrm{n}}}{p_{\mathrm{n}}} S\left(n_{3}\left(t_{1}\right), n_{3}\left(t_{2}\right)\right)\right) \\
& +\frac{e^{-\beta t_{1}}}{\beta} S\left(n_{3}\left(t_{1}\right), n_{3}\left(t_{2}\right)\right)+\int_{t_{2}}^{t_{1}} e^{-\beta t} S\left(n_{3}(t), n_{3}\left(t_{2}\right)\right) d t,
\end{aligned}
$$

where $\bar{\rho}=\frac{(1-\theta) \pi_{\mathrm{o}}}{\pi_{\mathrm{n}}}$.

Further, the first derivative of (A183) with respect to the adoption time $t_{1}$ is

$$
\begin{aligned}
\frac{\partial \widehat{\Pi}_{1}}{\partial t_{1}}= & \rho\left(\int_{t_{1}}^{+\infty} e^{-\beta t} \frac{\partial o_{13}(t)}{\partial t_{1}} d t\right)+\left(\int_{t_{1}}^{+\infty} e^{-\beta t} \frac{\partial S\left(n_{3}(t), n_{3}\left(t_{1}\right)\right)}{\partial t_{1}} d t\right) \\
= & (1-\rho) e^{-\beta t_{1}} \frac{d n_{3}\left(t_{1}\right)}{d t_{1}} \frac{p_{\mathrm{n}}}{p_{\mathrm{n}}+q_{\mathrm{n}} n_{3}\left(t_{1}\right)} \int_{0}^{+\infty} d u e^{-\beta u}\left(1+\frac{Q_{\mathrm{n}}}{p_{\mathrm{n}}} S\left(n_{3}(u), n_{3}\left(t_{1}\right)\right)\right) \\
& \times\left(\frac{n_{3}\left(t_{1}\right)-n_{3}(u)-S\left(n_{3}(u), n_{3}\left(t_{1}\right)\right)\left(2+\frac{Q_{\mathrm{n}}}{p_{\mathrm{n}}} S\left(n_{3}\left(t_{1}\right), n_{3}\left(t_{2}\right)\right)\right)}{1-S\left(n_{3}\left(t_{1}\right), n_{3}\left(t_{2}\right)\right)-n_{3}\left(t_{1}\right)}\right) \\
& \left.-\rho e^{-\beta t_{1}} \frac{d n_{3}\left(t_{1}\right)}{d t_{1}} \frac{p_{\mathrm{n}}}{p_{\mathrm{n}}+q_{\mathrm{n}} n_{3}\left(t_{1}\right)}\right) \\
& \times \int_{0}^{+\infty} d u e^{-\beta u}\left(\frac{1-S\left(n_{3}\left(t_{1}\right), n_{3}\left(t_{2}\right)\right)-n_{3}(u)-S\left(n_{3}(u), n_{3}\left(t_{1}\right)\right)\left(2+\frac{Q_{\mathrm{n}}}{p_{\mathrm{n}}} S\left(n_{3}\left(t_{1}\right), n_{3}\left(t_{2}\right)\right)\right.}{1-S\left(n_{3}\left(t_{1}\right), n_{3}\left(t_{2}\right)\right)-n_{3}\left(t_{1}\right)}\right) \\
& \times\left(1+\frac{Q_{\mathrm{n}}}{p_{\mathrm{n}}} S\left(n_{3}(u), n_{3}\left(t_{1}\right)\right)+\frac{p_{\mathrm{n}}+q_{\mathrm{n}} n_{3}(u)}{p_{\mathrm{n}}}\right),
\end{aligned}
$$

where we have used (A141) and (A142). Similarly, the first derivative of (A185) with respect to $t_{2}$ is given by

$$
\frac{\partial \widehat{\Pi}_{2}}{\partial t_{2}}=\bar{\rho} \frac{\partial \bar{\Pi}_{\mathrm{o}}\left(t_{1}, t_{2}\right)}{\partial t_{2}}+\frac{\partial \bar{\Pi}_{\mathrm{n}}\left(t_{1}, t_{2}\right)}{\partial t_{2}}\left(1+\frac{Q_{\mathrm{n}}}{p_{\mathrm{n}}} S\left(n_{3}\left(t_{1}\right), n_{3}\left(t_{2}\right)\right)\right)
$$




$$
\begin{aligned}
& +\left(\bar{\Pi}_{\mathrm{n}}\left(t_{1}, t_{2}\right) \frac{Q_{\mathrm{n}}}{p_{\mathrm{n}}}+\frac{e^{-\beta t_{1}}}{\beta}\right)\left(1+\frac{Q_{\mathrm{n}}}{p_{\mathrm{n}}} S\left(n_{3}\left(t_{1}\right), n_{3}\left(t_{2}\right)\right)\right) \frac{d n_{3}\left(t_{2}\right)}{d t_{2}} \\
& \times\left(\frac{p_{\mathrm{n}}}{p_{\mathrm{n}}+q_{\mathrm{n}} n_{3}\left(t_{1}\right)} \frac{d n_{3}\left(t_{1}\right)}{d n_{3}\left(t_{2}\right)}-\frac{p_{\mathrm{n}}}{p_{\mathrm{n}}+q_{\mathrm{n}} n_{3}\left(t_{2}\right)}\right) \\
& +\frac{d n_{3}\left(t_{2}\right)}{d t_{2}} \int_{t_{2}}^{t_{1}} e^{-\beta t}\left(\frac{p_{\mathrm{n}}}{p_{\mathrm{n}}+q_{\mathrm{n}} n_{3}(t)} \frac{d n_{3}(t)}{d n_{3}\left(t_{2}\right)}-\frac{p_{\mathrm{n}}}{p_{\mathrm{n}}+q_{\mathrm{n}} n_{3}\left(t_{2}\right)}\right) \\
& \times\left(1+\frac{Q_{\mathrm{n}}}{p_{\mathrm{n}}} S\left(n_{3}(t), n_{3}\left(t_{2}\right)\right)\right) d t .
\end{aligned}
$$

Further,

$$
\begin{aligned}
& \frac{\partial \bar{\Pi}_{\mathrm{o}}\left(t_{1}, t_{2}\right)}{\partial t_{2}}=\int_{t_{2}}^{t_{1}} e^{-\beta t} \frac{\partial o_{12}(t)}{\partial t_{2}} d t+\int_{t_{1}}^{+\infty} e^{-\beta t} \frac{\partial o_{13}(t)}{\partial t_{2}} d t=\frac{d n_{3}\left(t_{2}\right)}{d t_{2}} \int_{t_{2}}^{t_{1}} d t e^{-\beta t} \\
& \times\left(-\frac{d n_{3}(t)}{d n_{3}\left(t_{2}\right)}-\left(\frac{p_{\mathrm{n}}}{p_{\mathrm{n}}+q_{\mathrm{n}} n_{3}(t)} \frac{d n_{3}(t)}{d n_{3}\left(t_{2}\right)}-\frac{p_{\mathrm{n}}}{p_{\mathrm{n}}+q_{\mathrm{n}} n_{3}\left(t_{2}\right)}\right)\left(1+\frac{Q_{\mathrm{n}}}{p_{\mathrm{n}}} S\left(n_{3}(t), n_{3}\left(t_{2}\right)\right)\right)\right) \\
& +\frac{d n_{3}\left(t_{2}\right)}{d t_{2}} \int_{t_{1}}^{+\infty} d t e^{-\beta t} \\
& \times\left(-1-\left(\frac{p_{\mathrm{n}}}{p_{\mathrm{n}}+q_{\mathrm{n}} n_{3}(t)}\right)\left(1+\frac{Q_{\mathrm{n}}}{p_{\mathrm{n}}} S\left(n_{3}(t), n_{3}\left(t_{2}\right)\right)\left(2+\frac{Q_{\mathrm{n}}}{p_{\mathrm{n}}} S\left(n_{3}\left(t_{1}\right), n_{3}\left(t_{2}\right)\right)\right)\right)\right. \\
& \times \frac{d n_{3}(t)}{d n_{3}\left(t_{1}\right)} \frac{d n_{3}\left(t_{1}\right)}{d n_{3}\left(t_{2}\right)}+\frac{d n_{3}\left(t_{2}\right)}{d t_{2}} \int_{t_{1}}^{+\infty} e^{-\beta t}\left(\frac{p_{\mathrm{n}}}{p_{\mathrm{n}}+q_{\mathrm{n}} n_{3}\left(t_{1}\right)} \frac{d n_{3}\left(t_{1}\right)}{d n_{3}\left(t_{2}\right)}\right) \\
& \times\left(2+\frac{Q_{\mathrm{n}}}{p_{\mathrm{n}}} S\left(n_{3}\left(t_{1}\right), n_{3}\left(t_{2}\right)\right)\right)\left(1+\frac{Q_{\mathrm{n}}}{p_{\mathrm{n}}} S\left(n_{3}(t), n_{3}\left(t_{1}\right)\right) d t\right. \\
& -\frac{d n_{3}\left(t_{2}\right)}{d t_{2}} \int_{t_{1}}^{+\infty} e^{-\beta t}\left(1+\frac{Q_{\mathrm{n}}}{p_{\mathrm{n}}} S\left(n_{3}(t), n_{3}\left(t_{1}\right)\right)\right) \\
& \times\left(\frac{p_{\mathrm{n}}}{p_{\mathrm{n}}+q_{\mathrm{n}} n_{3}\left(t_{1}\right)} \frac{d n_{3}\left(t_{1}\right)}{d n_{3}\left(t_{2}\right)}-\frac{p_{\mathrm{n}}}{p_{\mathrm{n}}+q_{\mathrm{n}} n_{3}\left(t_{2}\right)}\right)\left(1+\frac{Q_{\mathrm{n}}}{p_{\mathrm{n}}} S\left(n_{3}\left(t_{1}\right), n_{3}\left(t_{2}\right)\right)\right) d t \\
& =-\frac{d n_{3}\left(t_{2}\right)}{d t_{2}} e^{-\beta t_{2}} \int_{0}^{+\infty} e^{-\beta u} \frac{d n_{3}(u)}{d n_{3}\left(t_{2}\right)} d t \\
& +\frac{d n_{3}\left(t_{2}\right)}{d t_{2}}\left(\frac{p_{\mathrm{n}}}{p_{\mathrm{n}}+q_{\mathrm{n}} n_{3}\left(t_{2}\right)}\right) e^{-\beta t_{2}} \int_{0}^{+\infty} e^{-\beta u}\left(1+\frac{Q_{\mathrm{n}}}{p_{\mathrm{n}}} S\left(n_{3}(u), n_{3}\left(t_{2}\right)\right) d u\right. \\
& -\frac{d n_{3}\left(t_{2}\right)}{d t_{2}} e^{-\beta t_{2}} \int_{0}^{+\infty} e^{-\beta u}\left(\frac{p_{\mathrm{n}}}{p_{\mathrm{n}}+q_{\mathrm{n}} n_{3}(u)} \frac{d n_{3}(u)}{d n_{3}\left(t_{2}\right)}\right)\left(1+\frac{Q_{\mathrm{n}}}{p_{\mathrm{n}}} S\left(n_{3}(u), n_{3}\left(t_{2}\right)\right) d u\right. \\
& -\frac{d n_{3}\left(t_{2}\right)}{d t_{2}} e^{-\beta t_{1}} \int_{0}^{+\infty} e^{-\beta u}\left(\frac{p_{\mathrm{n}}}{p_{\mathrm{n}}+q_{\mathrm{n}} n_{3}(u)} \frac{d n_{3}(u)}{d n_{3}\left(t_{1}\right)} \frac{d n_{3}\left(t_{1}\right)}{d n_{3}\left(t_{2}\right)}\right) \\
& \times\left(1+\frac{Q_{\mathrm{n}}}{p_{\mathrm{n}}} S\left(n_{3}(u), n_{3}\left(t_{2}\right)\right)\left(1+\frac{Q_{\mathrm{n}}}{p_{\mathrm{n}}} S\left(n_{3}\left(t_{1}\right), n_{3}\left(t_{2}\right)\right)\right) d u\right.
\end{aligned}
$$




$$
+\frac{d n_{3}\left(t_{2}\right)}{d t_{2}} e^{-\beta t_{1}} \int_{0}^{+\infty} e^{-\beta u}\left(\frac{p_{\mathrm{n}}}{p_{\mathrm{n}}+q_{\mathrm{n}} n_{3}\left(t_{1}\right)} \frac{d n_{3}\left(t_{1}\right)}{d n_{3}\left(t_{2}\right)}\right)\left(1+\frac{Q_{\mathrm{n}}}{p_{\mathrm{n}}} S\left(n_{3}(u), n_{3}\left(t_{1}\right)\right) d t\right.
$$

with

$$
\frac{d n_{3}\left(t_{1}\right)}{d n_{3}\left(t_{2}\right)}=\frac{\left(p_{\mathrm{n}}+q_{\mathrm{n}} n_{3}\left(t_{1}\right)\right)\left(1-n_{3}\left(t_{1}\right)-S\left(n_{3}\left(t_{1}\right), n_{3}\left(t_{2}\right)\right)\right)}{\left(p_{\mathrm{n}}+q_{\mathrm{n}} n_{3}\left(t_{2}\right)\right)\left(1-n_{3}\left(t_{2}\right)\right)},
$$

and

$\frac{d n_{3}(u)}{d n_{3}\left(t_{1}\right)}=\frac{\left(p_{\mathrm{n}}+q_{\mathrm{n}} n_{3}(u)\right)\left(1-n_{3}(u)-S\left(n_{3}\left(t_{1}\right), n_{3}\left(t_{2}\right)\right)-S\left(n_{3}(u), n_{3}\left(t_{1}\right)\right)\left(2+\frac{Q_{\mathrm{n}}}{p_{\mathrm{n}}} S\left(n_{3}\left(t_{1}\right), n_{3}\left(t_{2}\right)\right)\right)\right)}{\left(p_{\mathrm{n}}+q_{\mathrm{n}} n_{3}\left(t_{1}\right)\right)\left(1-n_{3}\left(t_{1}\right)-S\left(n_{3}\left(t_{1}\right), n_{3}\left(t_{2}\right)\right)\right)}$,

for $u \geq 0$. Also,

$$
\begin{aligned}
\frac{\partial \bar{\Pi}_{\mathrm{n}}\left(t_{1}, t_{2}\right)}{\partial t_{2}}= & \frac{d n_{3}\left(t_{1}\right)}{d n_{3}\left(t_{2}\right)} \frac{d n_{3}\left(t_{2}\right)}{d t_{2}} e^{-\beta t_{1}} \int_{0}^{+\infty} e^{-\beta u}\left(\frac{p_{\mathrm{n}}}{p_{\mathrm{n}}+q_{\mathrm{n}} n_{3}(u)} \frac{d n_{3}(u)}{d n_{3}\left(t_{1}\right)}-\frac{p_{\mathrm{n}}}{p_{\mathrm{n}}+q_{\mathrm{n}} n_{3}\left(t_{1}\right)}\right) \\
& \times\left(\frac{Q_{\mathrm{n}}}{p_{\mathrm{n}}} S\left(n_{3}(u), n_{3}\left(t_{1}\right)\right)+1\right) d u .
\end{aligned}
$$

We would like to establish conditions under which $\frac{\partial \widehat{\Pi}_{1}}{\partial t_{1}}\left(t_{1}, t_{2}\right) \leq 0$ for all $t_{1} \geq t_{2}$. Now, according to (A186) the sign of $\frac{\partial \widehat{\Pi}_{1}}{\partial t_{1}}$ is the same as the sign of

$$
\begin{aligned}
& (1-\rho) \int_{0}^{+\infty} d u e^{-\beta u}\left(1+\frac{Q_{\mathrm{n}}}{p_{\mathrm{n}}} S\left(n_{3}(u), n_{3}\left(t_{1}\right)\right)\right) \\
& \times\left(n_{3}\left(t_{1}\right)-n_{3}(u)-S\left(n_{3}(u), n_{3}\left(t_{1}\right)\right)\left(2+\frac{Q_{\mathrm{n}}}{p_{\mathrm{n}}} S\left(n_{3}\left(t_{1}\right), n_{3}\left(t_{2}\right)\right)\right)\right) \\
& -\rho \int_{0}^{+\infty} d u e^{-\beta u}\left(1+\frac{Q_{\mathrm{n}}}{p_{\mathrm{n}}} S\left(n_{3}(u), n_{3}\left(t_{1}\right)\right)\right) \\
& \times\left(1-S\left(n_{3}\left(t_{1}\right), n_{3}\left(t_{2}\right)\right)-n_{3}(u)-S\left(n_{3}(u), n_{3}\left(t_{1}\right)\right)\left(2+\frac{Q_{\mathrm{n}}}{p_{\mathrm{n}}} S\left(n_{3}\left(t_{1}\right), n_{3}\left(t_{2}\right)\right)\right)\right. \\
& -\beta \frac{\rho}{p_{\mathrm{n}}} \int_{0}^{+\infty} d u e^{-\beta u}\left(n_{3}(u)-n_{3}\left(t_{1}\right)\right) \\
& \leq(1-\rho) \int_{0}^{+\infty} d u e^{-\beta u}\left(1+\frac{Q_{\mathrm{n}}}{p_{\mathrm{n}}} S\left(n_{3}(u), n_{3}\left(t_{1}\right)\right)\right) \\
& \times\left(n_{3}\left(t_{1}\right)-n_{3}(u)-S\left(n_{3}(u), n_{3}\left(t_{1}\right)\right)\left(2+\frac{Q_{\mathrm{n}}}{p_{\mathrm{n}}} S\left(n_{3}\left(t_{1}\right), n_{3}\left(t_{2}\right)\right)\right)\right),
\end{aligned}
$$


which is non-positive for $\rho \leq 1$. In a similar fashion, $\bar{\rho} \leq 1$ implies that $\frac{\partial \widehat{\Pi}_{2}}{\partial t_{2}}\left(t_{1}, t_{2}\right) \leq 0$ for all $t_{2} \geq t_{1}$. The two conditions together imply that

$$
\frac{\partial \widehat{\Pi}_{1}}{\partial t_{1}}(0,0) \leq 0, \frac{\partial \widehat{\Pi}_{2}}{\partial t_{2}}(0,0) \leq 0
$$

so that the adoption Nash equilibrium is $t_{1}^{*}=t_{2}^{*}=0$.

b) Now,

$$
\frac{\partial \widehat{\Pi}_{1}}{\partial t_{1}}=\frac{d n_{3}\left(t_{1}\right)}{d t_{1}} \frac{p_{\mathrm{n}} e^{-\beta t_{1}} X\left(n_{3}\left(t_{1}\right), n_{3}\left(t_{2}\right)\right)}{\left(p_{\mathrm{n}}+q_{\mathrm{n}} n_{3}\left(t_{1}\right)\right)\left(1-S\left(n_{3}\left(t_{1}\right), n_{3}\left(t_{2}\right)\right)-n_{3}\left(t_{1}\right)\right)},
$$

where $\frac{d n_{3}\left(t_{1}\right)}{d t_{1}} \geq 0,1-S\left(n_{3}\left(t_{1}\right), n_{3}\left(t_{2}\right)\right)-n_{3}\left(t_{1}\right)>0$ and

$$
\begin{aligned}
X(a, b)= & (1-\rho) \int_{0}^{+\infty} d u e^{-\beta u}\left(1+\frac{Q_{\mathrm{n}}}{p_{\mathrm{n}}} S\left(n_{3}(u), a\right)\right)\left(a-n_{3}(u)-S\left(n_{3}(u), a\right)\left(2+\frac{Q_{\mathrm{n}}}{p_{\mathrm{n}}} S(a, b)\right)\right) \\
& -\rho \int_{0}^{+\infty} d u e^{-\beta u}\left(1+\frac{Q_{\mathrm{n}}}{p_{\mathrm{n}}} S\left(n_{3}(u), a\right)+\frac{p_{\mathrm{n}}+q_{\mathrm{n}} n_{3}(u)}{p_{\mathrm{n}}}\right) \\
& \times\left(1-S(a, b)-n_{3}(u)-S\left(n_{3}(u), a\right)\left(2+\frac{Q_{\mathrm{n}}}{p_{\mathrm{n}}} S(a, b)\right)\right) .
\end{aligned}
$$

Further, in order to compute the limit of $t_{1}=t_{\max }$ and $t_{2}=t_{\max }$, we set $a=b$ and let $a=1-\varepsilon$ with $\varepsilon<<1$. Then, starting at $n_{3}(0)=a$, and noting that $n_{3}(u)-a$ is of the order of $\varepsilon$ for all $u \geq 0$, we get

$$
S\left(n_{3}(u), a\right)=\frac{p_{\mathrm{n}}}{Q_{\mathrm{n}}}\left(\left(\frac{p_{\mathrm{n}}+q_{\mathrm{n}} n_{3}(u)}{p_{\mathrm{n}}+q_{\mathrm{n}} a}\right)^{\frac{Q_{\mathrm{n}}}{q_{\mathrm{n}}}}-1\right)=\frac{p_{\mathrm{n}} \bar{n}_{3}(u)}{p_{\mathrm{n}}+q_{\mathrm{n}} a}+o(\varepsilon),
$$

where

$$
\bar{n}_{3}(u)=n_{3}(u)-a .
$$

Denoting $\widehat{n}_{3}(u)=\frac{\bar{n}_{3}(u)}{p_{\mathrm{n}}+q_{\mathrm{n}}}$, we have the evolution equation for $\widehat{n}_{3}(u)$ as

$$
\frac{d \widehat{n}_{3}}{d u}=\left(1+\widehat{n}_{3}\right)\left(\varepsilon-\left(3 p_{\mathrm{n}}+q_{\mathrm{n}}\right) \widehat{n}_{3}(u)\right)=\varepsilon-\left(3 p_{\mathrm{n}}+q_{\mathrm{n}}\right) \widehat{n}_{3}(u),
$$

where we have only kept terms linear in $\varepsilon$. Then,

$$
\widehat{n}_{3}(u)=\frac{\varepsilon}{3 p_{\mathrm{n}}+q_{\mathrm{n}}}\left(1-e^{-\left(3 p_{\mathrm{n}}+q_{\mathrm{n}}\right) u}\right),
$$


so that

$$
n_{3}(u)=a+\frac{\varepsilon\left(p_{\mathrm{n}}+q_{\mathrm{n}}\right)}{3 p_{\mathrm{n}}+q_{\mathrm{n}}}\left(1-e^{-\left(3 p_{\mathrm{n}}+q_{\mathrm{n}}\right) u}\right),
$$

and

$$
S\left(n_{3}(u), a\right)=\frac{\varepsilon p}{3 p_{\mathrm{n}}+q_{\mathrm{n}}}\left(1-e^{-\left(3 p_{\mathrm{n}}+q_{\mathrm{n}}\right) u}\right),
$$

where, as before, we neglected terms of the order $o(\varepsilon)$. Combining these results, we get

$$
\lim _{a \rightarrow 1} X(a, a)=\left[(\rho-1) \frac{q_{\mathrm{n}}+3 p_{\mathrm{n}}}{\beta}-\rho\left(2+\frac{q_{\mathrm{n}}}{p_{\mathrm{n}}}\right)\right] \frac{\varepsilon}{\beta+3 p_{\mathrm{n}}+q_{\mathrm{n}}} \geq 0
$$

iff $\beta<\frac{p_{\mathrm{n}}\left(q_{\mathrm{n}}+3 p_{\mathrm{n}}\right)}{q_{\mathrm{n}}+2 p_{\mathrm{n}}}$ and $\rho \geq \frac{1}{1-\frac{\beta\left(2 p_{\mathrm{n}}+q_{\mathrm{n}}\right)}{p_{\mathrm{n}}\left(3 p_{\mathrm{n}}+q_{\mathrm{n}}\right)}}$. In a similar way, $\frac{\partial \widehat{\Pi}_{2}}{\partial t_{2}}$ is positive for high values of both $t_{1}$ and $t_{2}$ iff $\beta<\frac{p_{\mathrm{n}}\left(q_{\mathrm{n}}+3 p_{\mathrm{n}}\right)}{q_{\mathrm{n}}+2 p_{\mathrm{n}}}$ and $\bar{\rho} \geq \frac{1}{1-\frac{\beta\left(2 p_{\mathrm{n}}+q_{\mathrm{n}}\right)}{p\left(3 p_{\mathrm{n}}+q_{\mathrm{n}}\right)}}$. Thus, both firms delay their adoption as much as possible when both $\rho$ and $\bar{\rho}$ exceed $\frac{1}{1-\frac{\beta\left(2 p_{n}+q_{\mathrm{n}}\right)}{p_{\mathrm{n}}\left(3 p_{\mathrm{n}}+q_{\mathrm{n}}\right)}}>1$, provided that, in addition, $\beta<\frac{p_{\mathrm{n}}\left(q_{\mathrm{n}}+3 p_{\mathrm{n}}\right)}{q_{\mathrm{n}}+2 p_{\mathrm{n}}}$.

c) We would like to establish sufficient conditions guaranteeing that $t_{1}^{*}=t_{\max }, t_{2}^{*}=0$. As it follows from (A194) and (A195), for the first incumbent we need to evaluate

$$
\lim _{a \rightarrow a^{*}} \frac{X(a, 0)}{1-S(a, 0)-a},
$$

where $1-S\left(a^{*}, 0\right)-a^{*}=0$. Now, consider $a$ such that $1-S(a, 0)-a=\varepsilon$ with $\varepsilon<<1$. Then, similarly to (A200) and (A201), we get

$$
n_{3}(u)=a+\frac{\varepsilon\left(p_{\mathrm{n}}+q_{\mathrm{n}} a^{*}\right)}{3 p_{\mathrm{n}}+q_{\mathrm{n}} a^{*}+Q_{\mathrm{n}} S\left(a^{*}, 0\right)}\left(1-e^{-\left(3 p_{\mathrm{n}}+q_{\mathrm{n}} a^{*}+Q_{\mathrm{n}} S\left(a^{*}, 0\right)\right) u}\right),
$$

and

$$
S\left(n_{3}(u), a\right)=\frac{\varepsilon p_{\mathrm{n}}}{3 p_{\mathrm{n}}+q_{\mathrm{n}} a^{*}+Q_{\mathrm{n}} S\left(a^{*}, 0\right)}\left(1-e^{-\left(3 p_{\mathrm{n}}+q_{\mathrm{n}} a^{*}+Q_{\mathrm{n}} S\left(a^{*}, 0\right)\right) u}\right) .
$$

Thus, keeping terms linear in $\varepsilon$, we get

$$
\lim _{a \rightarrow a^{*}} X(a, 0)=\left[(\rho-1) \frac{3 p_{\mathrm{n}}+q_{\mathrm{n}} a^{*}+Q_{\mathrm{n}} S\left(a^{*}, 0\right)}{\beta}-\rho\left(2+\frac{q_{\mathrm{n}} a^{*}}{p_{\mathrm{n}}}\right)\right] \frac{\varepsilon}{\beta+3 p_{\mathrm{n}}+q_{\mathrm{n}} a^{*}+Q_{\mathrm{n}} S\left(a^{*}, 0\right)} \geq 0
$$

if and only if $\beta<\frac{p_{\mathrm{n}}\left(q_{\mathrm{n}} a^{*}+Q_{\mathrm{n}} S\left(a^{*}, 0\right)+3 p_{\mathrm{n}}\right)}{q_{\mathrm{n}} a^{*}+2 p_{\mathrm{n}}}$ and $\rho \geq \frac{1}{1-\frac{\beta\left(2 p_{\mathrm{n}}+q_{\mathrm{n}} a^{*}\right)}{p_{\mathrm{n}}\left(3 p_{\mathrm{n}}+q_{\mathrm{n}} a^{*}+Q_{\mathrm{n}} S\left(a^{*}, 0\right)\right)}}$. Further, in the case of $t_{1} \rightarrow+\infty$, the first-order condition for the adoption time of the second incumbent is

$$
\frac{\partial \widehat{\Pi}_{2}}{\partial t_{2}}=\bar{\rho} \frac{\partial \bar{\Pi}_{\mathrm{o}}\left(t_{1}, t_{2}\right)}{\partial t_{2}}-\int_{0}^{+\infty} e^{-\beta u}\left(p_{\mathrm{n}}+Q_{\mathrm{n}} S\left(n_{3}(u), 0\right)\right)
$$




$$
\begin{aligned}
& \times\left(n_{3}(u)+S\left(a^{*}, 0\right)+S\left(n_{3}(u), a^{*}\right)\left(2+\frac{Q_{\mathrm{n}}}{p_{\mathrm{n}}} S\left(a^{*}, 0\right)\right)\right) d u \\
\leq & \int_{0}^{+\infty} e^{-\beta u} d u\left(p_{\mathrm{n}}+Q_{\mathrm{n}} S\left(n_{3}(u), 0\right)\right) \\
& \times\left(\bar{\rho}\left(n_{3}(u)-S\left(n_{3}(u), 0\right)\right)-n_{3}(u)-S\left(a^{*}, 0\right)-S\left(n_{3}(u), a^{*}\right)\left(2+\frac{Q_{\mathrm{n}}}{p_{\mathrm{n}}} S\left(a^{*}, 0\right)\right)\right) \leq 0,
\end{aligned}
$$

for $\bar{\rho} \leq 1$. 


\section{Appendix B: Estimates of FX Trading Volumes and Their Sources}

Below we report the data and its sources for the FX example we used to estimate the diffusion parameters.

In particular, Table B1 shows the estimates for the average daily trading volumes for the "new entrant", Currenex, while Tables B2 and B3 show these for the Atriax ("major" incumbent) and the FXAll ( "minor" incumbent).

\begin{tabular}{|c|c|c|}
\hline Date & Average Daily Trading Volume, \$bn & Source \\
\hline Oct. 2000 & 0.5 & Reuters News, 07/13/2001 \\
\hline Jul. 2001 & 1.0 & Reuters News, 07/13/2001 \\
\hline Jul. 2002 & 1.5 & Reuters News, 07/15/2002 \\
\hline Apr. 2003 & 3.5 & Reuters News, 04/14/2003 \\
\hline Feb. 2004 & 4.5 & Dow Jones International News, 02/20/2004 \\
\hline Mar. 2005 & 5.0 & Routers News, 03/02/2005 \\
\hline Feb. 2007 & 40.0 & Gones Capital Markets, $02 / 26 / 2007$ \\
\hline Nov. 2010 & 250.0 & Global Finance, 01/01/2011 \\
\hline
\end{tabular}

Table B1 The average daily trading volumes for the Currenex: estimates and their sources.

\begin{tabular}{|c|c|c|}
\hline Date & Average Daily Trading Volume, $\$$ bn & Source \\
\hline Oct. 2001 & 0.22 & Financial News, 10/29/2001 \\
\hline Apr. 2002 & $0.4^{*}$ & American Banker, 04/26/2002 \\
\hline
\end{tabular}

Table B2 The average daily trading volumes for the Atriax: estimates and their sources. *The estimate for Apr. 2002 was obtained by averaging the lower estimate $(0.3 \mathrm{bn})$ and the upper estimate (0.5bn) 


\begin{tabular}{|c|c|c|}
\hline Date & Average Daily Trading Volume, $\$$ bn & Source \\
\hline Aug. 2001 & 0.2 & Reuters News, 03/15/2002 \\
\hline Apr. 2002 & 1.5 & Reuters News, 05/04/2002 \\
\hline Jan. 2003 & 4 & Reuters News, 04/14/2003 \\
\hline Apr. 2003 & 7.5 & Reuters News, 04/14/2003 \\
\hline Jul. 2003 & 10.6 & Reuters News, 07/09/2003 \\
\hline Nov. 2003 & 13.0 & Financial Times, 12/03/2003 \\
\hline Mar. 2004 & 18.0 & Financial Times, 04/14/2004 \\
\hline Oct. 2004 & 25.0 & Financial Times, $10 / 28 / 2004$ \\
\hline Dec. 2004 & 30.0 & Reuters News, 01/10/2005 \\
\hline Mar. 2005 & 35.0 & Reuters News, 03/14/2005 \\
\hline Oct. 2005 & 43.0 & Reuters News, 10/10/2005 \\
\hline May. 2006 & 54.0 & Reuters News, 05/05/2006 \\
\hline Nov. 2006 & 64.0 & Reuters News, 11/06/2006 \\
\hline Oct. 2007 & 90.0 & Reuters News, 10/04/2007 \\
\hline Mar. 2010 & 100.0 & Dow Jones, 03/26/2010 \\
\hline Nov. 2010 & 125.0 & Global Finance, 01/01/2011 \\
\hline
\end{tabular}

Table B3 The average daily trading volumes for the FXAll: estimates and their sources. 